\title{
Holographic Polymer-Dispersed Liquid Crystals: Materials, Formation, and Applications
}

\author{
Y. J. Liu and X. W. Sun \\ School of Electrical and Electronic Engineering, Nanyang Technological University, Nanyang Avenue, Singapore 639798 \\ Correspondence should be addressed to X. W. Sun, exwsun@ntu.edu.sg
}

Received 29 August 2008; Accepted 30 November 2008

Recommended by H. Kwok

\begin{abstract}
By combining polymer-dispersed liquid crystal (PDLC) and holography, holographic PDLC (H-PDLC) has emerged as a new composite material for switchable or tunable optical devices. Generally, H-PDLC structures are created in a liquid crystal cell filled with polymer-dispersed liquid crystal materials by recording the interference pattern generated by two or more coherent laser beams which is a fast and single-step fabrication. With a relatively ideal phase separation between liquid crystals and polymers, periodic refractive index profile is formed in the cell and thus light can be diffracted. Under a suitable electric field, the light diffraction behavior disappears due to the index matching between liquid crystals and polymers. H-PDLCs show a fast switching time due to the small size of the liquid crystal droplets. So far, H-PDLCs have been applied in many promising applications in photonics, such as flat panel displays, switchable gratings, switchable lasers, switchable microlenses, and switchable photonic crystals. In this paper, we review the current state-of-the-art of H-PDLCs including the materials used to date, the grating formation dynamics and simulations, the optimization of electro-optical properties, the photonic applications, and the issues existed in H-PDLCs.
\end{abstract}

Copyright ( $) 2008$ Y. J. Liu and X. W. Sun. This is an open access article distributed under the Creative Commons Attribution License, which permits unrestricted use, distribution, and reproduction in any medium, provided the original work is properly cited.

\section{Introduction}

\subsection{Polymer and Photopolymerization}

The discovery of polymers has much contributed to the change of day-to-day life. In fact, the use of polymers in human history dates back as early as Egyptian mummies. Before the mummies were wrapped, the cloth was soaked in a solution of oil lavender. When the cloth was dried by exposure to the sunlight, the oil was converted into a protective polymer. This was a typical application of photopolymerization. Polymers are involved in almost all aspects of our lives. Some examples include their use in textile, automotive and electronic industries, and as building and packing materials.

Monomers are the building blocks or structural units of the polymers. For polymer formation, the monomer must be able to link two or more other monomers, that is, the functionality of the monomer is not less than 2 . Depending on the functionalities of the monomer, different morphologies of polymer networks can be formed [1]. If the polymer is constructed from difunctional monomers, it is a linear chain (Figure 1(a)). If higher functional monomers are used, a branched polymer will be formed (Figure 1(b)). The presence of the branches limits the motion of chains, thus strengthening the polymer. If the functionality is even higher, the branches start to connect to one another in three dimensions. The polymer becomes a cross-linked network (Figure 1(c)). This cross-linked network enables the polymer to be much stronger, but also much more brittle.

The most widely used and simple method of polymer formation is radical-chain addition polymerization. Polymerization involves initiation, propagation, transfer, and termination steps. Initiation is the step where initiating radicals are formed by external stimulations such as light, heat, gamma-radiation, and redox processes. Lightinduced photopolymerization has several advantages over other methods. The fabrication temperature is low, usually room temperature, and controllability can be realized by selecting different irradiation wavelength and light intensity. 


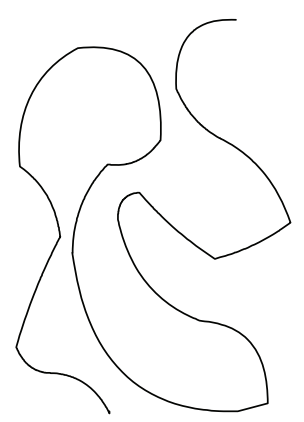

(a)

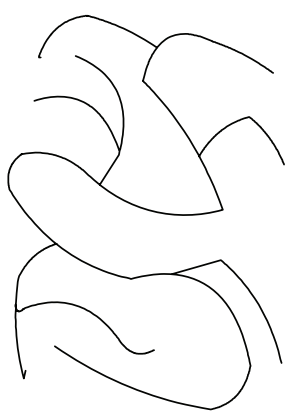

(b)

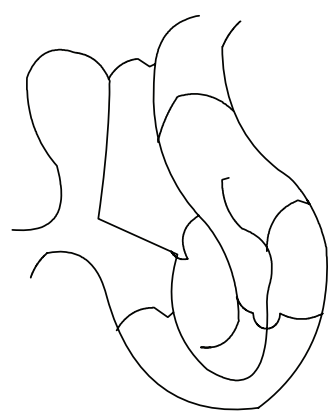

(c)

Figure 1: Different polymer structures. (a) A linear polymer chain. (b) A branched chain. (c) A cross-linked network.

Moreover, light can be focused on a particular site, thus polymer can be formed where it is desired.

Photopolymerization is a very flexible process and can be used in various technological applications such as photoresists in modern electronics. In this process, a film of monomer mixture is placed on the surface of a silicon wafer and is illuminated through a photomask. After polymerization, the unpolymerized material can be washed away, leaving the polymer intact. The polymer-covered areas on the silicon wafer are then used to resist chemical etching, resulting in integrated circuit formation or printed circuit board construction. This technology has allowed billions of transistors to be placed on a single integrated circuit.

\subsection{Liquid Crystal and Polymer Composites}

In the past decade, liquid crystal/polymer composites have attracted great attention due to their special dynamic characteristics. Liquid crystal/polymer composites could appear in the form of network or droplet depending on the polymer and its concentration. In the low-concentration regime (1$2 \mathrm{wt} \%)[2,3]$, the response time can be much improved, however, the associated light scattering is quite strong in the visible range. As the polymer concentration increases to 3-8 $\mathrm{wt} \%$, the polymer-stabilized liquid crystal (PSLC, also known as gel) is formed in a homogeneous or homeotropic cell $[4,5]$. These PSLC cells exhibit an anisotropic light scattering behavior and have potential for reflective display and telecom applications $[6,7]$. As the polymer concentration increases to $30-40 \mathrm{wt} \%$, the polymer-dispersed liquid crystal (PDLC) is formed $[8,9]$. No surface alignment layers in the PDLC cell are needed. Micron-sized LC droplets are buried in the polymer matrix. In this case, visible light is strongly scattered and this scattering is independent of polarization. In the high polymer concentration regime (60$70 \mathrm{wt} \%$ ), nanoscale holographic PDLC (H-PDLC) droplets are formed $[10,11]$. Since the droplet size is much smaller than the visible wavelength, H-PDLC film is free of light scattering and its response time is fast (30-200 $\mu$ s). However, to reorient LC molecules in these small droplets, a relatively high switching electric field $(15-20 \mathrm{~V} / \mu \mathrm{m})$ is required. In this approach, no surface alignment is required.

\subsection{Holography and H-PDLC}

Holography is the process of recording the complete information of the electric field in both amplitude and relative phase, and consequently a real three-dimensional image can be reconstructed $[12,13]$. Since most of recording materials are only sensitive to the intensity of the light, interference is a very convenient way to convert the phase information to the amplitude information. Transmission and reflection holograms are two common types whose images can be reconstructed by appropriate use of optics, such as prisms, lenses, and mirrors. The most attractive and unique property of a hologram is the wavelength and angle sensitivity, and the ability to manipulate the light that is either reflected or diffracted. To date, holographic optical elements (HOEs) have found numerous important roles in photonics.

Photopolymers are one of the holographic materials that have been widely used in the fabrication of HOEs. Thin volume gratings can be obtained by holographic interference patterning and subsequent modulation of the refractive index in photopolymer materials. They can be classified into two categories, active and passive devices, depending on their optical function. An element that can change its refractive index dynamically with applied external stimulus, such as electric field, optical strength, forces, is called an active optical element, while an element with a fixed modulation of the refractive index is called a passive optical element.

The marriage of holography and PDLC materials [14] has produced a number of photonic applications in information and display systems, electro-optic filters, free-space optical switches, and wavelength division multiplexer. For a typical PDLC, LCs are randomly distributed in the polymer matrix and the droplets are randomly orientated, while in H-PDLC system, the interference of two beams generates periodic dark and bright fringes in the photopolymer film. In the photopolymer system, the polymerization rate is higher in the bright region and lower in the dark region. During the holographic recording process, the monomer diffuses into the bright region to form polymer and at the same time LCs diffuse into dark regions. The main advantage of this technique is the fast and single-step ability to fabricate large area grating structures. 


\subsubsection{Transmission and Reflection Gratings}

Depending on the writing geometries, H-PDLC gratings can be divided into two different modes according to the holography recording configuration, transmission, and reflection. For transmission H-PDLC, the recording two beams are in the same side of the medium, as shown in Figure 2(a). In transmission H-PDLC, the recorded fringes are perpendicular to the substrates. This type of H-PDLC is very sensitive to the incident angle of light beam. On the contrary, for reflecting H-PDLC, the recording light beams are in the two sides of the recording medium, indicated as Figure 2(b). In reflection H-PDLC, the recorded fringes are parallel to the substrates. This type of H-PDLC is very sensitive to the wavelength. Therefore, we can design the grating pitch to reflect red, green, or blue light, which makes it potentially useful in displays. Here, for both reflection and transmission H-PDLCs, the recording light beams are all symmetrical, and therefore the fringes are parallel or perpendicular to the substrates. If the recording beams are not symmetrical, slant fringes will be obtained in H-PDLC.

\subsubsection{Raman-Nath and Bragg Gratings}

Generally, two types of diffraction are distinguished by defining a dimensionless Cook-Klein parameter Q:

$$
Q=\frac{2 \pi \lambda d}{n \Lambda^{2}},
$$

where $d$ is the thickness of the grating, $\Lambda$ is the grating period, $n$ is the spatially averaged refractive index of the recording medium, and $\lambda$ is the incident wavelength.

A "thick" or volume grating $(Q \geq 10)$ corresponds to the Bragg diffraction and provides the diffraction when incident angle satisfies the phase matching conditions. Such structures exhibit only two diffraction orders (zeroth and first order) and a strong dependency of the diffraction efficiency on the angle and wavelength of the incident light. The diffraction efficiency of the transmission volume phase grating in Bragg conditions is described by

$$
\eta_{\mathrm{vol}}=\sin ^{2}\left(\frac{\Delta \delta_{\mathrm{vol}}}{2}\right)=\sin ^{2}\left(\frac{\pi n_{1} d}{\lambda \cos \theta_{0}}\right),
$$

where $n_{1}$ is the amplitude of refractive index modulation of the material, $\theta_{0}$ is the Bragg angle of the incident light within the material.

On the other hand, "thin" gratings $(Q \leq 1)$ correspond to the Raman-Nath regime of the optical diffraction. In this regime, many orders of the diffraction can be observed. The efficiency of the first-order Raman-Nath diffraction for the grating with a sinusoidal modulation of refractive index is given by

$$
\eta_{\text {surf }}=J_{1}^{2}\left(\frac{\Delta \delta_{\text {surf }}}{2}\right)=J_{1}^{2}\left(\frac{2 \pi h\left(n_{I}-n_{I I}\right)}{\lambda}\right),
$$

where $J_{1}^{2}\left(\Delta \delta_{\text {surf }} / 2\right)$ is the first-order Bessel function of the first kind, $h$ is the depth of the gratings grooves, $n_{I}$ and $n_{I I}$ are the refractive indices of the adjacent areas of the grating.

\subsection{POLYCRYPS}

Recently, a new kind of holographic grating made of polymer slices alternated to films of regularly aligned nematic liquid crystals, so-called POLICRYPS, an acronym of Polymer Liquid Crystal Polymer Slices, was demonstrated by an Italian research group [15-18]. This kind of grating was fabricated at an elevated temperature, which is higher than the nematic-isotropic transition temperature, thus avoiding the appearance of the nematic phase during the curing process. After cooling, these structures present a sharp and uniform morphology, without those optical inhomogeneities which are due to the NLC droplets in usual H-PDLC samples. As a result, the scattering losses were reduced and the diffraction efficiency was increased accordingly. The diffraction efficiency of POLYCRYPS was reported to be as high as $98 \%$ [18]. The big difference between H-PDLC and POLYCRYPS gratings was shown in Figure 3. Based on POLYCRYPS gratings, Asquini et al. developed a switchable optical add-drop multiplexer [19]. This designed switchable add-drop multiplexer using a POLICRYPS grating performs with a cross-talk of $-22 \mathrm{~dB}$ and with just $0.8 \mathrm{~nm}$ wavelength spacing as used in DWDM optical communication systems. However, compared to most H-PDLCs, the POLICRYPS grating shows more sensitivity to the polarization and slower response times including rising time and falling time [20].

\subsection{H-PDFLCs}

Ferroelectric liquid crystals (FLCs) have been demonstrated as excellent candidates for fast electro-optic switching applications such as displays [21] and optical switches [22]. However, limited device commercialization is available, partly due to the lack of achieving suitable alignment layers that are both stable and robust for homogeneous alignment of the FLC. Over the past decade, researchers have shown different techniques to aid in the alignment of ferroelectric liquid crystals by combining FLCs and polymer dispersions with shearing forces [23]. Recently, some researchers investigated the holographic polymerdispersed ferroelectric liquid crystals (H-PDFLCs) [24-26]. In H-PDFLCs, different alignment states of FLCs can be created by changing the weight ratio of FLCs and monomer. Figures 4(a) and 4(b) show the droplet-like and channel-like morphologies in the FLC-rich regions for H-PDFLC samples with a 50:50 weight ratio mixture of FLC and monomer and a 40:60 weight ratio mixture, respectively, which show different polarization properties. The droplet-like samples produced polarization insensitive while the channel-like ones produced polarization sensitive electro-optical properties, which indicated different alignments of FLCs in these two different kinds of samples. In the droplet-like samples, the FLCs are not well aligned, inducing the lack of grating contrast under a polarized optical microscope, as shown in Figure 5. While in the channel-like samples, the FLCs are well aligned, which produces a sharp grating contrast under the polarized optical microscope, as shown in Figure 6. Woltman and his coworkers developed a phenomenological model to describe the channel-like morphology of the homogeneously 


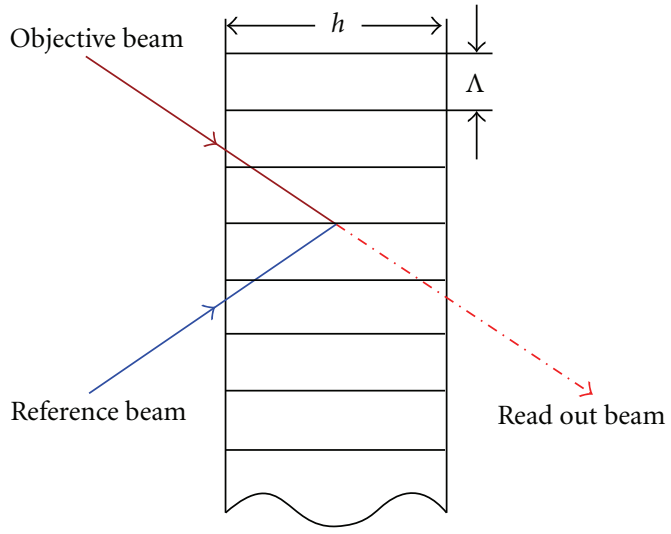

(a)

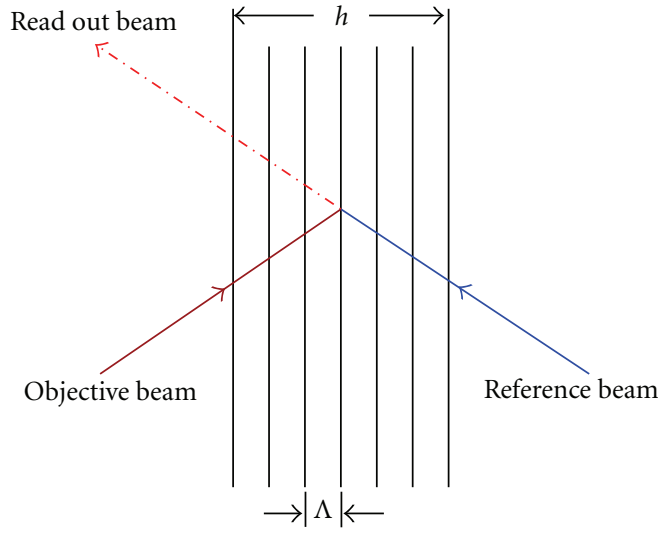

(b)

FIGURE 2: Schematic holographic recording for (a) transmission and (b) reflection gratings, respectively.

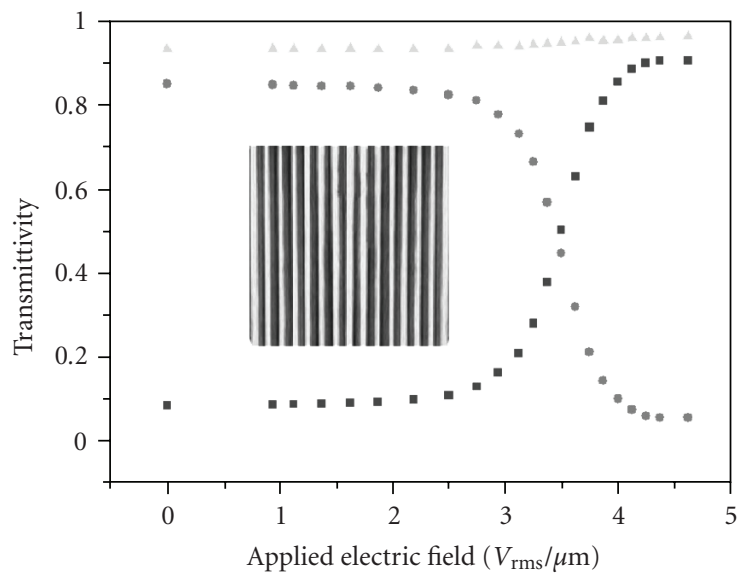

(a)

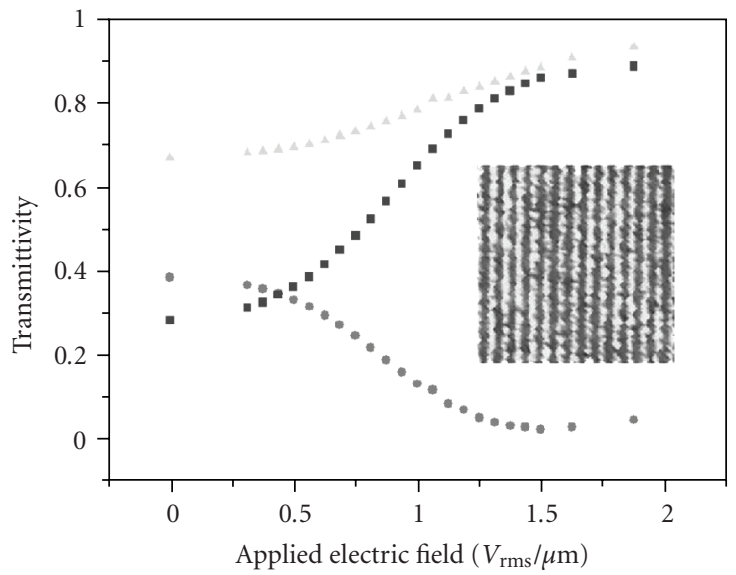

(b)

FIGURE 3: Field dependence of transmission curve (squares), first-order diffraction curve (circles), and total transmission curve (triangles) for (a) a POLICRYPS grating, (b) a PDLC grating. The insets show typical (a) POLICRYPS, (b) PDLC grating morphology with the same period, observed under a polarizing optical microscope [18].

aligned FLCs between the polymer walls [26]. The FLC alignment is parallel to the polymer walls, which is different with the nematic-based H-PDLCs, where the nematic LC alignment is orthogonal to the polymer walls [27]. The $\mathrm{H}-$ PDFLCs studies are still in early stage and the results are preliminary. A big advantage of H-PDFLCs is the faster response time. Rapid switching between 20 and $50 \mu$ s [27] has been observed for the H-PDFLCs above a threshold voltage.

\subsection{Azo Dye Doped H-PDLC}

In some cases, polarization sensitive gratings are useful as a polarized or unpolarized beam splitter, depending on the polarization of the incident light. Adding a small amount of azo dye into the PDLC materials, polarization sensitive gratings can be obtained by holography [28]. The formation of the polarization gratings is attributed to the anisotropic adsorption of the azo dyes on the surfaces of polymer walls, inducing the reorientation of the liquid crystal molecules [29]. Figure 7 shows a sharp grating contrast under an optical microscope with a polarizer parallel and perpendicular to the direction of the stripe in the grating, respectively. Woo et al., also investigated the effect of the azo dye on the diffraction efficiency, gratings morphologies, and electro-optic properties [30]. As the concentration of dye molecules increased, the liquid crystal droplets became small due to the slow rate of polymerization, as shown in Figure 8. In addition, azo dye molecules strongly reorient LC molecules perpendicular to the grating, giving rise to enhanced diffraction efficiency and short response times.

\subsection{Nematic Director Configurations in PDLCs}

A typical example of PDLCs is the light valve configuration presented in Figure 9. The PDLC light valve is fabricated by 


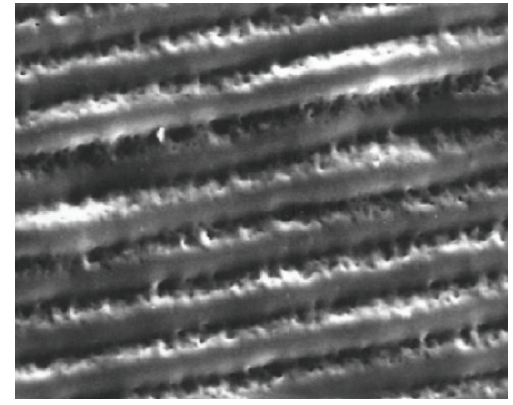

(a)

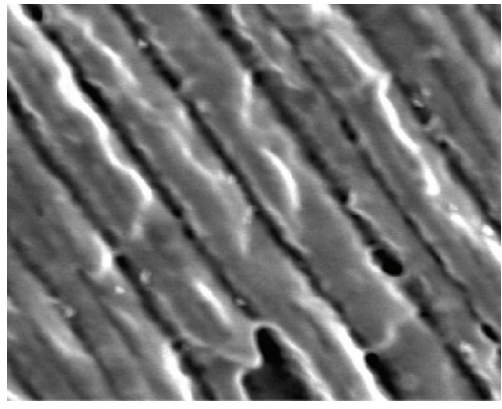

(b)

Figure 4: H-PDFLC samples constructed from (a) a 50:50 weight mixture of FLC and monomer, and from (b) a 40:60 weight mixture, respectively [24].

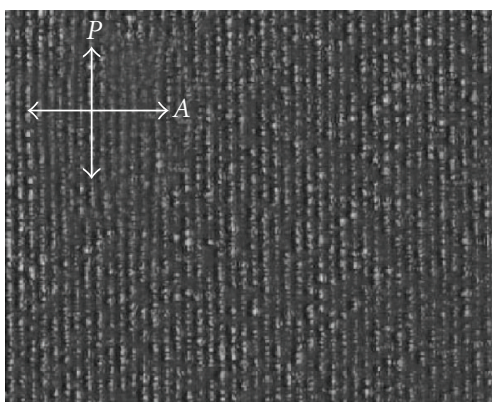

(a)

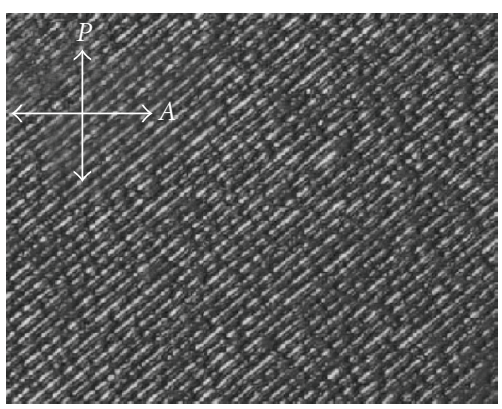

(b)

FIGURE 5: Optical polarizing microscopy of a polarization insensitive FLC H-PDLC grating (a) $90^{\circ}$ between crossed polarizers, (b) $45^{\circ}$ between crossed polarizers [25].

sandwiching PDLC material between transparent conducting electrodes with a $10-25 \mu \mathrm{m}$ spacer. In the "off" state, the liquid crystal droplets with positive dielectric anisotropy are randomly oriented in the film and the size of the droplets is comparable to or larger than an optical wavelength, usually $2-5 \mu \mathrm{m}$. In the translucent state, the film has a white opaque appearance due to the scattering properties of the film. Once an electric field is applied, the liquid crystal molecules in each droplet will align in the direction parallel to the field direction as shown in Figure 9 ("on" state). If the ordinary refractive index, $n_{\mathrm{o}}$, (perpendicular to the nematic director) approximately matches the refractive index, $n_{\mathrm{p}}$, of the surrounding polymer matrix, the film will be transparent. Upon removal of the field, the droplets will return to their random orientation and the film reverts back to its opaque state. Therefore, the PDLCs are switched between the scattering state and transmission state.

The PDLC structure formation through photopolymerization is a complicated process including physical changes and chemical reactions. Usually, the droplets of a nearly uniform size are formed during the phase separation. The average droplet size varies from submicron up to several tens of microns depending on the conditions during the formation process. The nematic structure inside a droplet is the result of the interplay between elastic, surface, and external field interactions. The minimization of the phenomenological free energy is usually used to determine the stable structure of a chosen system at constant temperature and volume. It is convenient to divide the free-energy density into elastic $f_{e}$, interfacial $f_{s}$, and field $f_{f}$ parts.

The nematic free-energy density $f_{e}$ can be expressed as

$$
\begin{aligned}
f_{e}=\frac{1}{2} & {\left[k_{11}(\nabla \cdot \mathbf{n})^{2}+k_{22}(\mathbf{n} \cdot \nabla \times \mathbf{n})^{2}+k_{33}(\mathbf{n} \times \nabla \times \mathbf{n})^{2}\right.} \\
& \left.-k_{24} \nabla \cdot(\mathbf{n}(\nabla \cdot \mathbf{n})+\mathbf{n} \times \nabla \times \mathbf{n})\right],
\end{aligned}
$$

where $k_{11}, k_{22}$, and $k_{33}$ are the splay, twist, and bend elastic constants, respectively, and $\mathbf{n}$ is the director axis.

The interaction of the liquid crystal with the surrounding medium is described by a simple contact interaction

$$
f_{s}=\left(1-\left(\mathbf{n} \cdot \mathbf{e}_{r}\right)^{2}\right) \frac{\mathbf{W}_{0}}{2} \delta(\mathbf{r}-\mathbf{R})
$$

characterized by $\mathbf{e}_{r}$, the preferred anchoring direction on the droplet surface and the anchoring strength $\mathbf{W}_{0}$. The vector $\mathbf{R}$ in (5) defines points on the droplet surface.

The interaction with an external magnetic field $\mathbf{B}$ is described by

$$
f_{f}=-\frac{\mu_{0}}{2} \Delta \chi(\mathbf{B} \cdot \mathbf{n})^{2},
$$

where $\mu_{0}$ is the permeability of free space, $\mathbf{B}$ is the external magnetic field, and $\Delta \chi$ is the difference between the principal values of the susceptibility tensor corresponding to the directions parallel and perpendicular to $\mathbf{n}$. 


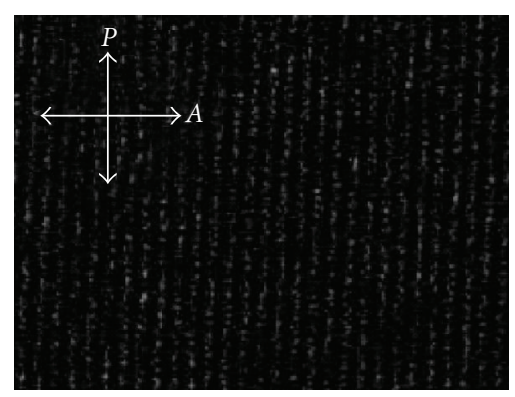

(a)

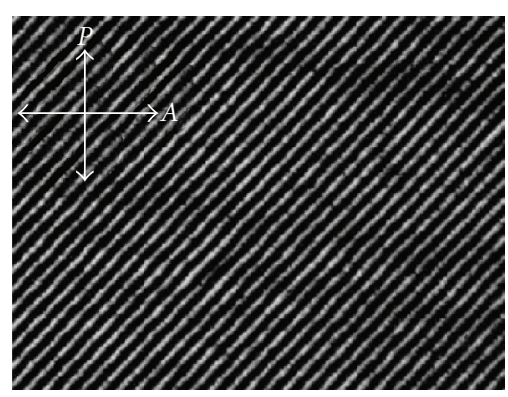

(b)

FIGURE 6: Optical polarizing microscopy of a polarization insensitive FLC H-PDLC grating (a) $90^{\circ}$ between crossed polarizers, (b) $45^{\circ}$ between crossed polarizers [25].

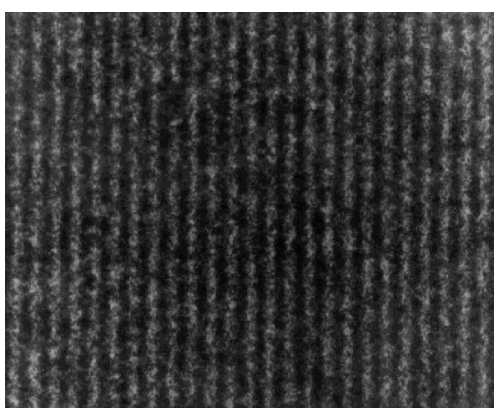

(a)

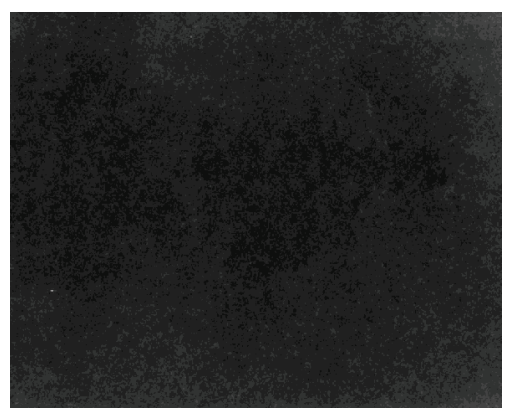

(b)

FIGURE 7: Grating images observed under an optical microscope with a polarizer placed in front of the sample. The transmission axis of the polarizer is (a) parallel and (b) perpendicular to the direction of the stripes in the grating [29].

The minimization of the total free energy

$$
\mathbf{F}=\int\left(f_{e}+f_{s}+f_{f}\right) \mathrm{d}^{3} \mathbf{r}
$$

is achieved by solving the Euler-Lagrange differential equations, which lead to the prediction of equilibrium structures. With very few exceptions, the solutions are obtained numerically using relaxation methods $[31,32]$.

The director field configurations of some equilibrium structures are schematically illustrated in Figure 10 for spherical and ellipsoid shaped cavities. Figures 10(a) and 10 (c) are two of the most commonly observed nematic director configurations resulted from parallel and perpendicular boundary conditions, respectively. The effect of an electric field on the bipolar configuration is to align the symmetry axis parallel to the field with little distortion of the director configuration within the droplet, as shown in Figure 10(b). The effect of the electric field on the radial configuration is to induce a configuration transition to the axial configuration shown in Figure 10(d). Particularly in large droplets, metastable structures are relatively diverse. Figures $10(\mathrm{e})-10(\mathrm{~h})$ have also been reported in some special cases $[33,34]$. The nematic director configurations in the liquid crystal droplets in PDLCs help to understand the similar things in H-PDLCs, although the droplets in $\mathrm{H}$ PDLCs are usually much smaller (less than $100 \mathrm{~nm}$ ) than those in PDLCs.
In the following parts of this review article, we will concentrate on the H-PDLCs. H-PDLCs have wide range applications for displays [35], switchable gratings [14, 3638], photonic crystals [39-41], lasers [42-45], microlenses $[46,47]$, and so forth. A vitally important method used to construct H-PDLC devices is photopolymerization induced phase separation (PIPS) by either UV or visible lasers, depending on the materials used. Different from the working mechanism of PDLC, H-PDLC works between the diffraction state and transmission state, as shown in Figure 11. By applying a proper electric field, the grating can be switched between "on" and "off" states. The working principle of such a switch is fairly straightforward. A linearly polarized light beam incident on the switch will see the phase grating formed by the LC-polymer materials with different refractive index, and thus be diffracted. When an electric field is applied, the LC molecular director is reoriented, changing the refractive index seen by the incoming light. If properly designed, with a suitable voltage applied, $n_{\mathrm{LC}}=n_{\text {polymer }}$ can be achieved, and the Bragg grating disappears. If and when the condition is met, the beam is no more diffracted.

\section{H-PDLC Materials}

One of the fundamentals of research on H-PDLCs is to develop new formulations of materials. All formulations have two underlying components: monomer and liquid crystal. 


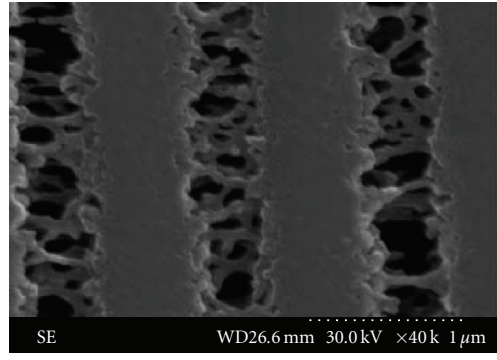

(a)

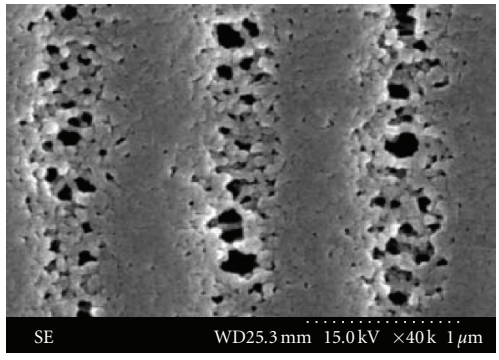

(b)

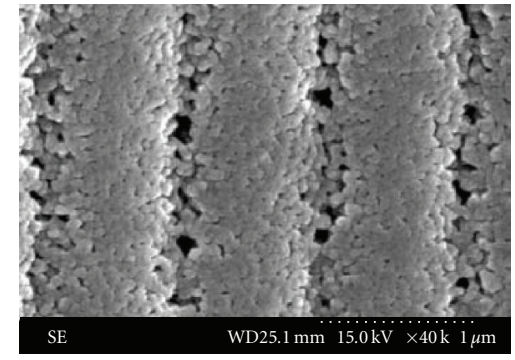

(c)

FIGURE 8: SEM micrographs for a transmission HPDLC prepared (a) without and with (b) $0.5 \%$, (c) $1 \%$ azo dye [30].

"OFF" state

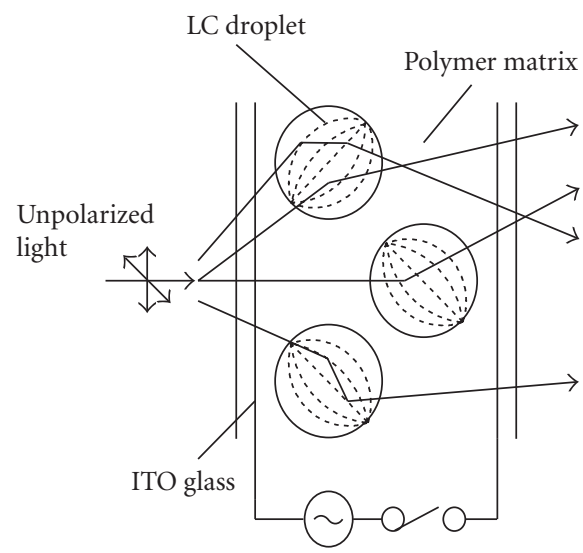

(a)
“ON" state

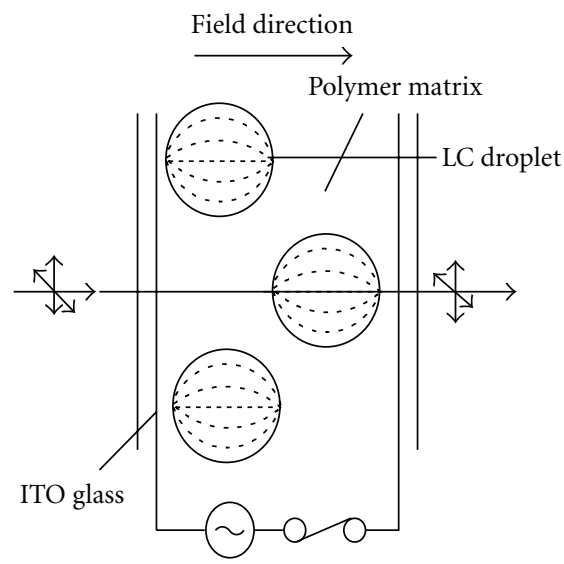

(b)

FIGURE 9: Illustration of the principle behind the operation of a PDLC display. The material has a white translucent appearance in the "off" state. Application of a voltage to the transparent conducting electrodes reorients the symmetry axis of the bipolar droplets parallel to the electric field direction ("on" state) and the material becomes transparent if the ordinary refractive index of the liquid crystal matches that of the polymer matrix.

In addition, to improve the performance of H-PDLCs, some other components may be added into the material set, such as photoinitiator dye, coinitiator, surfactant, and so on. Both the ratios of these components and the components themselves will determine the performance of H-PDLC.

\subsection{Ultraviolet (UV) Curing}

The general material formulation for a UV-curing prepolymer mixture consists of the thiol-ene photopolymers, NOA series (Norland Inc.), and photoinitiator, Irgacure 1173, which is developed by Natarajan and his coworkers, specially for the wavelength of $363 \mathrm{~nm}$ from $\mathrm{Ar}^{+}$laser [48]. NOA 65 is the most common one in NOA series, which is widely used in photonics for adhesion of optical components. Although the commercial NOA 65 has a proprietary UV initiator, the addition of Irgacure 1173 UV initiator will greatly enhance the diffraction efficiency.

The advantages of such kind of material system compared to the acrylate system are smaller shrinkage, more stable electro-optical properties, and long-term stability. The droplets of LC are bigger than those in acrylate system, leading to a decreased driving voltage. However, the response time is relatively long, in the order of milliseconds.

\subsection{Visible (Blue and Green) Curing}

The above NOA series materials can be also polymerized under visible light exposure if another kind of photoinitiator is doped into these materials. The reflection mode $\mathrm{H}$ PDLC grating was made using NOA 81 doped Rose Bengal (RB) under a $532 \mathrm{~nm}$ laser exposure by Wu and Fuh [49]. However, generally, for such kind of materials, high exposure intensity is needed to ensure a good structure grating formation.

In the visible range, the acrylate material system is the most common one used to date, which cures at $488 / 514.5 \mathrm{~nm}$ produced by $\mathrm{Ar}^{+}$laser or $532 \mathrm{~nm}$ produced by a frequencydoubled Nd:YAG laser. Sutherland et al., firstly fabricated the Bragg grating using acrylate materials [14].

The most common recipe of the H-PDLC for the visible curing generally contains a multifunctional monomer, chain 
extender N-vinyl pyrrolidinone (NVP), photoinitiator RB, coinitiator N-phenylglycine (NPG), surfactant, and nematic liquid crystal. Different monomers with different functionalities were tried in this kind of formulation, such as Dipentaerythritol penta-/hexa-acrylate (DPPHA), pentaerythritol triacrylate (PETA), trimethylolpropane triacrylate (TMPTA), pentaerythritol tetraacrylate [PET(etra)A], and Tri(propylene glycol) diacrylate (TPGDA). Their chemical structures were shown in Figure 12. The chemical structures of RB, NPG, and NVP are shown in Figure 13. The advantages of the photoinitiator (RB) chosen are that it has a broad absorption spectrum in the region of $470-$ $550 \mathrm{~nm}$ (as indicated in Figure 14) and it is very suitable for excitation with argon ion laser wavelengths as well as frequency-doubled Nd-YAG (532 nm) [50].

\subsection{Visible (Red) Curing}

Given the abundance and low cost of He-Ne lasers and red diode lasers, the feasibility investigation of recording a $\mathrm{H}$ PDLC grating by utilizing $633 \mathrm{~nm}$ wavelength of an $\mathrm{He}-\mathrm{Ne}$ laser is hence motivated. Ramsey and Sharma firstly reported $\mathrm{H}-\mathrm{PDLC}$ grating fabrication using $\mathrm{He}-\mathrm{Ne}$ laser $[51,52]$. The big significance is to make H-PDLC to be fabricated using a very small power laser and lower the cost of the fabrication.

The material system for He-Ne laser curing is composed of monofunctional acrylic oligomer, CN135, cross-linker, SR295, and initiator (photo-oxidant dye), methylene blue and coinitiator, $\mathrm{p}$-toluenesulfonic acid.

\subsection{Near Infrared (NIR) Curing}

The development of H-PDLC material systems for NIR curing also attracts much attention without exception. Pilot et al. developed the material system under NIR range in 1999 [53]. The materials used include monomer, Di-pentaerithrithol-penta-acrylate (DPEPA) doped with 2-ethoxyethoxy-ethyl acrylate ester (2EEEA), Cyanine dye, IR-140, initiator, ethyl-di-methyl-amino-benzoate (EDMABzt), and electron donor, CBr4. An 834 or $850 \mathrm{~nm}$ laser diode was used to illuminate the material. The material can achieve good resolution (2500 lines/mm) and shows excellent long-term chemical stability. However, same as the acrylate system, the switching voltage is still relatively high.

\section{Fabrication}

The prepolymer and LC were mechanically blended according to the appropriate weight ratio and stirred in an ultrasonic cleaner at a temperature higher than the clearing point of the liquid crystal for at least 2 hours to form a homogeneous mixture in dark. After that, the mixture was injected in a cell by capillary effect or in vacuum, which was formed by two pieces of indium-tin-oxide (ITO) coated glass, and then subjected to laser exposure. The cell thickness can be controlled by the bead spacers between the two pieces of ITO glass. During exposure, the pattern formed by two interfering beams was recorded in the LC cell. After exposure,

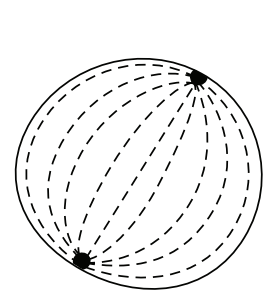

(a)

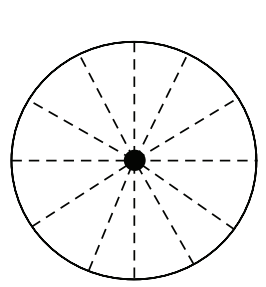

(c)

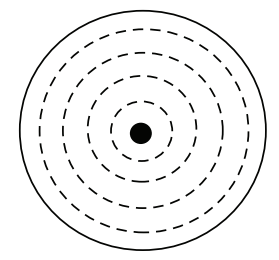

(e)

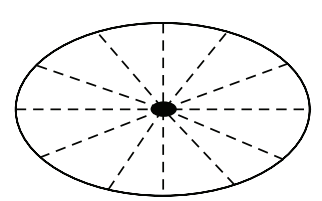

(g)

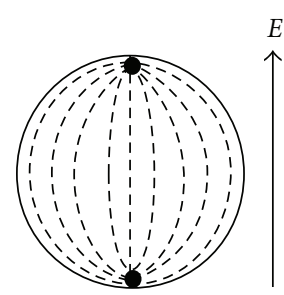

(b)

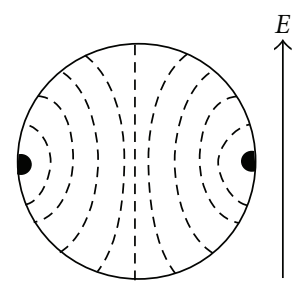

(d)

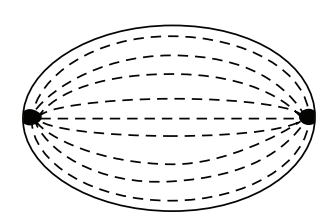

(f)

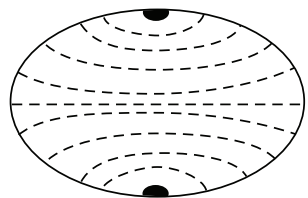

(h)
FIgURE 10: Schematic illustration of nematic director configurations in spherical cavities: (a) the bipolar configuration, (b) the bipolar configuration aligned by an electric field, (c) the radial configuration, (d) the axial configuration in an applied field, and (e) the toroidal configuration. The corresponding (f) bipolar, (g) radial, and (h) axial configurations in elongated droplets.

the samples were further cured for 15 minutes by mercury lamp to ensure the complete polymerization of prepolymer.

Depending on the writing geometries, transmission and reflection mode gratings can be fabricated, respectively. In the case of writing transmission gratings, the writing beams are coincident on the same side of the sample with an angle between the two writing beams and the resulting grating vector is parallel to the substrates. In reflection gratings, the writing beams are counter propagating and the resulting grating vector is perpendicular to the substrates. Figure 15 shows the specially designed setups to fabricate the transmission and reflection gratings, respectively. The use of single prism in the fabrication setups decreases the complexity of the optics. More importantly, the setups are self-adaptive, that is, the effect of the external vibration during the fabrication process is minimized. The LC cells filled with prepolymer mixture stick onto the hypotenuse of the prism using the index matching liquid. 


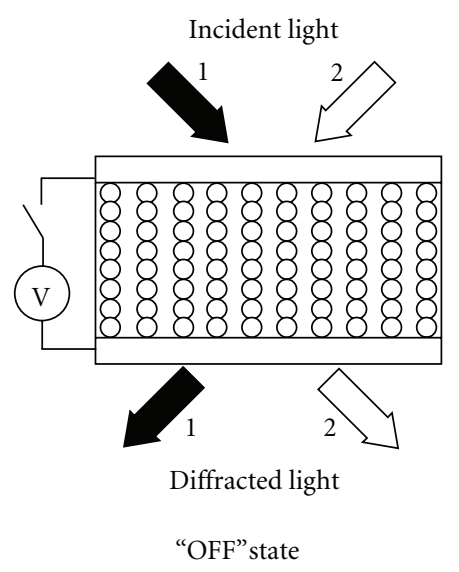

(a)

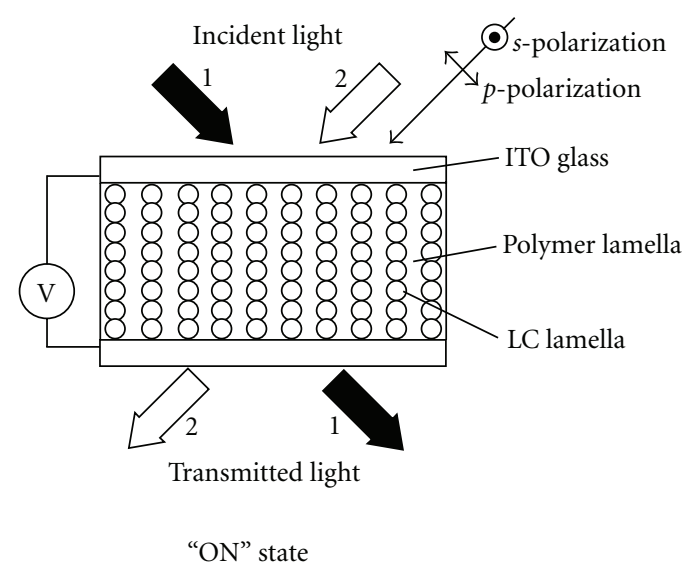

(b)

Figure 11: Schematic of an H-PDLC transmission grating.

\section{Characterization}

\subsection{Structures}

For morphology analysis, the samples were fractured first by sinking them in liquid nitrogen. Then the samples with oneside ITO glass removed are soaked in ethanol for more than 12 hours in order to completely remove the LC. After drying, the morphologies can be investigated by high-resolution optical microscope, atomic force microscopy (AFM), or scanning electron microscope (SEM).

AFM (Dimension 3000 Scanning Probe Microscope, Digital Instruments) is used to measure the surface morphology of the films. The root mean square (RMS) roughness is obtained from the digital image-processing package of the AFM system.

Field emission gun scanning electron microscopy (FEGSEM) (JEOL JSM 6700F) is used to observe the surface morphology of the films. Since the H-PDLC films are nonconductive polymers, a thin layer of gold or platinum is coated on the H-PDLC surface by a JEOL JFC-1200 fine coater in order to eliminate the charging effect.

\subsection{Electro-Optical Properties}

\section{(i) Diffraction Efficiency}

For the switchable volume gratings, diffraction efficiency is a very important parameter. It is defined as the diffracted power in the first order divided by the incident power. All samples were measured with polarized He-Ne laser $(\lambda=$ $633 \mathrm{~nm}$ ) at room temperature. The diffraction efficiency for $p$ - and $s$-polarized light was measured and compared, respectively.

\section{(ii) Response Time}

Figure 16 shows the measurement setup of the response time. Polarized light from a He-Ne laser was used for the diffraction/transmittance properties and the response time studies. The laser beam was incident on the sample with the exact Bragg angle, and the diffraction was measured by a photomultiplier. In the measurements, the H-PDLC Bragg grating was driven by an AC signal with various frequencies. In our measurement, a high-voltage amplifier/function generator (Trek, Model: 609E-6-FG) is used to generate the AC signal with different waveforms and frequencies. For the response time measurements, the photodetector signal was fed into one input channel of an oscilloscope (Agilent, Model: 54641A). The voltage signal, which was used to trigger the oscilloscope, was fed into another input channel of the oscilloscope. From the waveforms captured by the oscilloscope, the rising and falling times can be subtracted.

The turn-on time is the time required for the transmitted intensity to change from $10 \%$ to $90 \%$ upon application of the voltage, and the turn-off time is the time required for the transmitted intensity to decrease from $90 \%$ to $10 \%$ after removal of the voltage. The switching time is the sum of the turn-on and turn-off times. Typically, the turn-on time is inversely proportional to the applied voltage and is limited by the viscosity of the liquid crystal. The turn-off time is typically inversely proportional to droplet size, and is governed by the relaxation processes.

The switching voltage depends on the droplet morphology and the electrical properties of the liquid crystal and the polymer matrix. Typically, the switching voltage is inversely proportional to the droplet size. Small droplets enhance the liquid crystal's elastic deformation energy, and thus larger voltages are required to reorient the liquid crystal molecules confined to the droplets and thus to switch on a PDLC. The enhanced elastic deformation energy associated with small droplets also leads to shorter turn-off times. The switching voltage also depends on the resistive properties of the liquid crystal droplets and surrounding polymer. For example, higher applied voltages are required if the resistive properties reduce the electric field experienced by the droplets. 


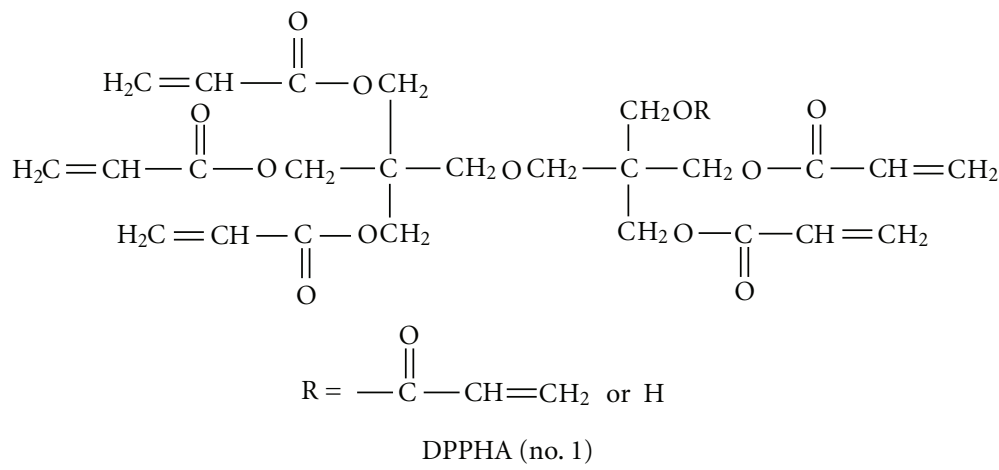<smiles>C=CC(=O)OCC1(CO)COC(=O)C(=C)C(=O)O1</smiles>

PETA (no. 2)<smiles>C=CC(=O)OCC(CC)(COC(=O)C=C)COC(=O)C=C</smiles>

TMPTA (no. 3)<smiles>C=CC(=O)OCC(COC(=O)C=C)(COC(=O)C=C)COC(=O)C=C</smiles>

PET(etra)A (no. 4)<smiles>C=COC(=O)CCCOC(=O)C=C</smiles>

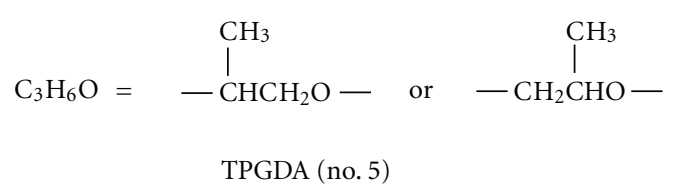

FIgURE 12: Chemical structures of the five monomers.

\section{Phase Separation Kinetics}

\subsection{Formation Kinetics of H-PDLCs}

The grating formation process is schemed in Figure 17. Grating formation ensues when the material is irradiated with a sinusoidal intensity pattern. In the bright regions, absorption by the photoinitiator results in a highly reactive triplet state. The coinitiator undergoes an electron-transfer reaction with this triplet state to create a free radical. Freeradical polymerization is then initiated. The presence of the chain-extender promotes cross-linking of the polymer chains produced. The result is a rapid consumption of monomers and polymer growth in the bright regions of the intensity pattern.

Ignoring interactions between various components, the chemical potential for the $i$ th component of the mixture may be approximated by

$$
\mu_{i}=\mu_{i}^{0}+k_{B} T \ln \left(\frac{N_{i}}{\sum_{j} N_{j}}\right),
$$

where $\mu_{i}^{0}$ is the chemical potential of the pure $i$ th component, $N_{i}$ is the number of molecules of the $i$ th component, $k_{B}$ is 
<smiles>COC(=O)c1c(Cl)c(Cl)c(Cl)c(Cl)c1-c1c2cc(I)c(=O)c(I)c-2oc2c(I)c(O[N+](=O)[O-])c(I)cc12</smiles>

$\mathrm{RB}$<smiles>O=C(O)CNc1ccccc1</smiles>

NPG<smiles>C=CN1CCCC1=O</smiles>

NVP

Figure 13: Chemical structures of H-PDLC components.

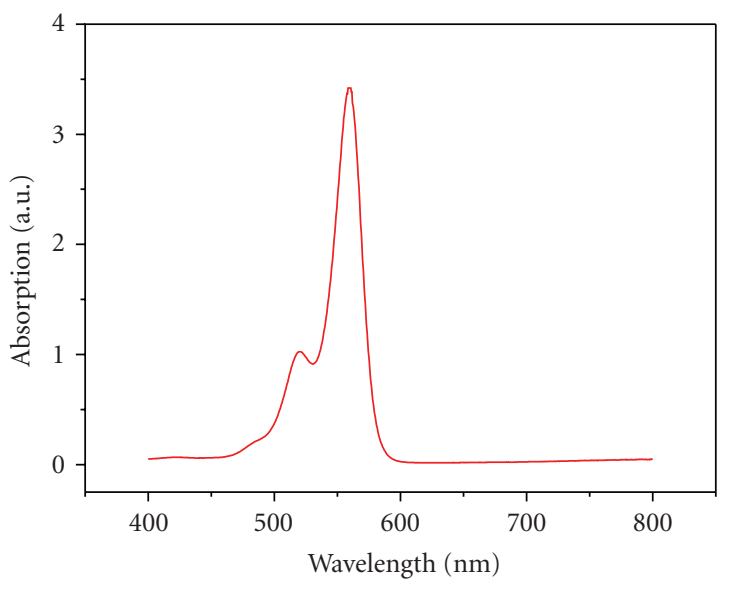

Figure 14: Absorption spectrum of the photoinitiator dye RB.

Boltzmann's constant, and $T$ is the absolute temperature. Under thermodynamic equilibrium, the sum $\mu_{i}$ is a constant. A gradient in the light intensity preferentially initiates photopolymerization in the high-intensity regions. Since the mixture is dominated by monomer and LC components initially, we see that the chemical potential of the monomer decreases while that of the LC increases in the bright regions. In the neighboring dark regions, at least for early times, the chemical potentials are all equal to their initial equilibrium values. Hence, spatial gradients in the chemical potential are established which produces a diffusion of monomers (and other reactants) into the bright regions, and a counterdiffusion of LC into the dark regions. This process continues until a new equilibrium chemical potential is established.

As the growing polymer network propagates outward into the dark regions, gelation of the system ensues. Fluctuations in the free energy of the system result in local minima where a distinct LC phase begins to form. Similar to the discussion of the droplet formation in PDLCs in Section 1.7, the total droplet energy is minimized at a critical droplet radius, $R c$, which is proportional to the surface tension of the droplet, $\sigma$. A droplet of this size will be stable and can grow if more LC molecules are available for incorporation or if coalescence with a nearby droplet is feasible. Growth will

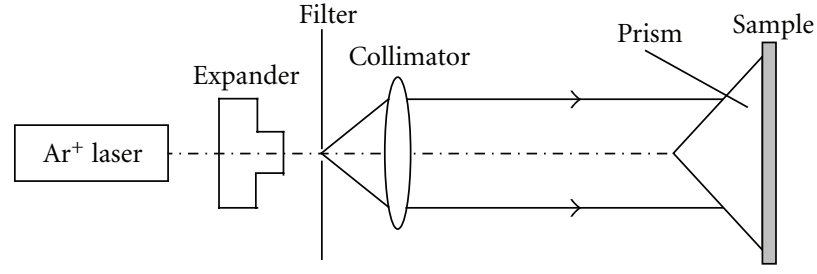

(a)

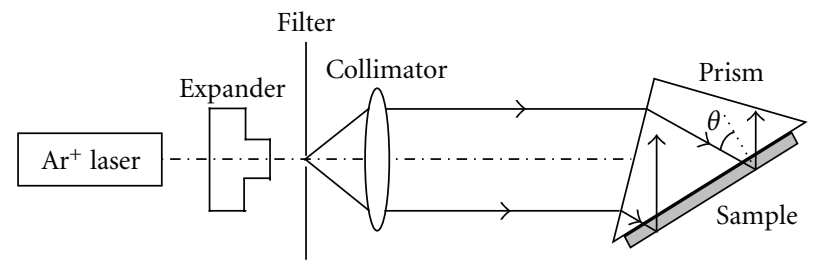

(b)

FIgURE 15: Experimental setups of holographic (a) transmission and (b) reflection grating recording, respectively.

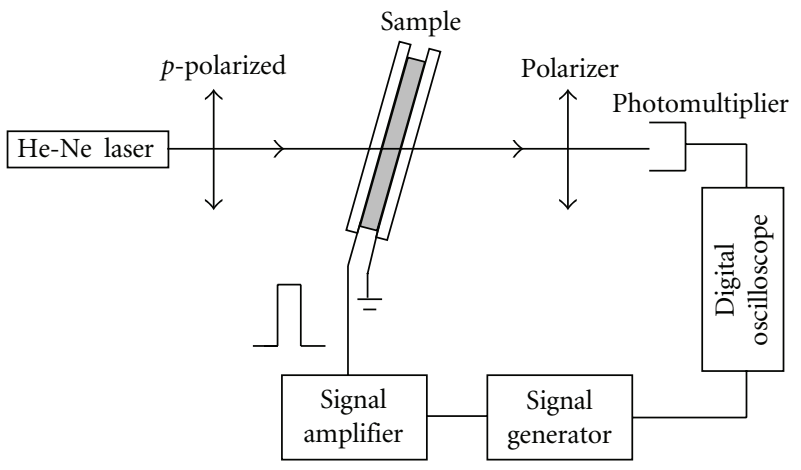

FIGURE 16: Measurement setup of the response time.

eventually terminate when the surrounding polymer rigidly locks the droplet in place and prevents further LC diffusion.

A big disadvantage of free-radical photopolymerization is its susceptibility to oxygen inhibition. This inhibition is particularly problematic in thin film and coating applications where oxygen diffusion plays a significant role in increasing 


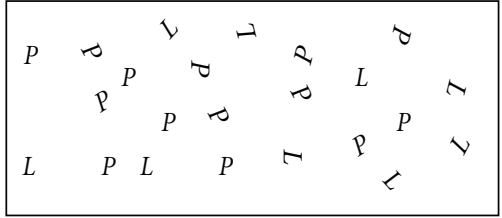

(a)
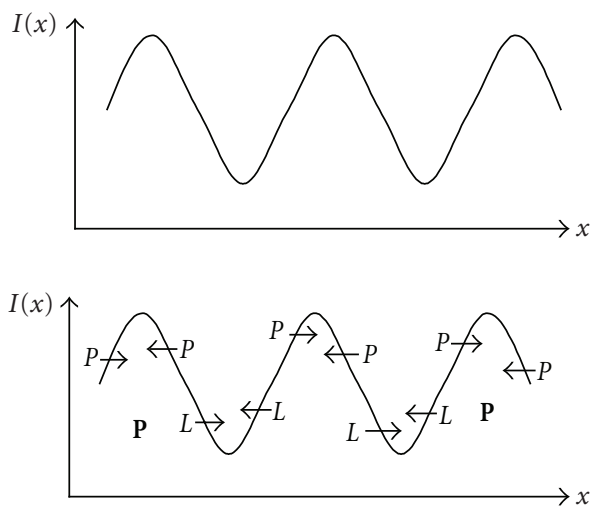

(b)

\begin{tabular}{|c|c|c|c|c|}
\hline $\mathrm{P}_{\mathrm{P}}$ & $L$ & $P$ & $L$ & P P \\
\hline $\mathbf{P}$ & $L$ & $\mathrm{P}^{\mathrm{P}}$ & L & P P \\
\hline$P^{P}$ & $\begin{array}{l}L \\
L\end{array}$ & ${ }_{P}{ }^{P}$ & $\begin{array}{l}L \\
L\end{array}$ & ${ }_{P} P$ \\
\hline${ }_{P}{ }^{P}$ & $I$ & $\mathrm{P}^{\mathrm{P}}$ & $L$ & ${ }_{\mathrm{p}} \mathrm{P}$ \\
\hline P P & $L$ & P P & $L$ & ${ }_{\mathrm{P}} \mathrm{P}$ \\
\hline
\end{tabular}

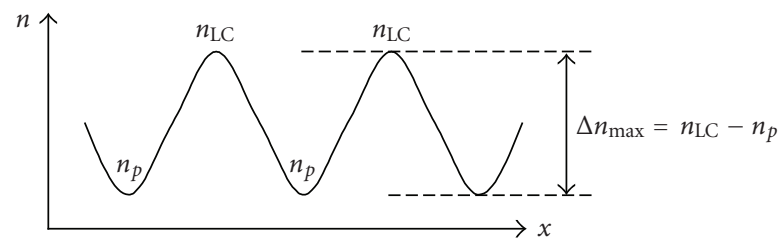

(c)

FIGURE 17: Schematic formation process of H-PDLC gratings. (a) Homogeneous mixture before exposure. (b) The exposure intensity distribution is sinusoidal. In the bright region, the monomer polymerizes first. The liquid crystal diffuses to the dark region. (c) Finally, alternate polymer-rich and liquid crystal region formed. The refractive index is modulated along $x$-axis direction.

cure times, which often results in incomplete conversion $[54,55]$. When oxygen, which is essentially a biradical in its electronic ground state, reacts with a free radical, it forms a peroxy radical, which is much less reactive.

As a result, the kinetic reactions of photo-induced freeradical polymerization can be summarized by the following four steps.

Initiation:

$$
\begin{gathered}
\mathrm{I} \stackrel{h v}{\longrightarrow} \mathrm{R}^{\bullet} \\
\mathrm{R}^{\bullet}+\mathrm{M}_{n} \stackrel{k_{i}}{\longrightarrow} \mathrm{M}_{n}^{\cdot} .
\end{gathered}
$$

Propagation:

$$
\mathrm{M}_{n}^{\bullet}+\mathrm{M} \stackrel{k_{p}}{\longrightarrow} \mathrm{M}_{n+1}^{\cdot}
$$

Termination:

$$
\begin{gathered}
\mathrm{M}_{n}^{\cdot}+\mathrm{M}_{m}^{\cdot} \stackrel{k_{t c}}{\longrightarrow} \mathrm{M}_{n+m} \\
\mathrm{M}_{n}^{\cdot}+\mathrm{M}_{m}^{\cdot} \stackrel{k_{t d}}{\longrightarrow} \mathrm{M}_{n}+\mathrm{M}_{m} .
\end{gathered}
$$

Inhibition by Oxygen:

$$
\begin{gathered}
\mathrm{M}^{\bullet}+\mathrm{O}_{2} \longrightarrow \mathrm{M}-\mathrm{O}-\mathrm{O}^{\bullet} \\
\mathrm{M}-\mathrm{O}-\mathrm{O}^{\bullet}+\mathrm{M} \longrightarrow \text { No Reaction. }
\end{gathered}
$$

\subsection{One-Dimensional Diffusion Model}

Physically, the formation of the H-PDLC gratings can be well simulated using one-dimensional (1D) diffusion model. Assuming the monomers are free to diffuse in the materials, a $1 \mathrm{D}$ diffusion equation can be written for the monomer concentration as follows [56, 57]:

$$
\frac{d \phi(x, t)}{d t}=-F(x, t) \phi(x, t)+\frac{d}{d x}\left(D(x, t) \frac{d \phi(x, t)}{d x}\right),
$$

where $\phi(x, t)$ is the monomer concentration, $F(x, t)$ is the polymerization rate, and $D(x, t)$ is the diffusion rate.

When monomers react and bind with the growing polymer radical, free volume is generated since the covalent single carbon bond in the polymer is as much as $50 \%$ shorter than the Van der Waals bond in the liquid monomer state. The mechanical response of the medium to reduce this free volume leads to physical shrinkage of the system. Assuming the volume shrinkage rate is $z$, which denotes that the monomers convert into $1 /(1+z)$ polymer in unit volume. Therefore, the concentration of the polymer can be written as

$$
\psi(x, t)=\int_{0}^{t} \frac{1}{1+z} F\left(x, t^{\prime}\right) \phi\left(x, t^{\prime}\right) d t^{\prime},
$$

the concentration of liquid crystal can be written as

$$
\begin{aligned}
\eta(x, t)=\int_{0}^{t} & {\left[\frac{z}{1+z} F\left(x, t^{\prime}\right) \phi\left(x, t^{\prime}\right)\right.} \\
& \left.-\frac{d}{d x}\left(D\left(x, t^{\prime}\right) \frac{d \phi\left(x, t^{\prime}\right)}{d x}\right)\right] d t^{\prime} .
\end{aligned}
$$

In the whole system, $\psi, \phi$, and $\eta$ are satisfying the equation $\psi+\phi+\eta=1$. So there are only two independent parameters in this system.

Consider a holographic grating formed by the interference of two plane waves in liquid crystal-polymer composites. The spatial distribution of optical intensities is

$$
I(x, t)=I_{0}[1+V \cos (k x)],
$$

where $I_{0}$ is the average intensities, $V$ is the fringe visibility, $k=2 \pi / \Lambda$, and $\Lambda$ is the grating pitch. We assume the polymerization rate to be proportional to the exposure intensities, so we may write

$$
F(x, t)=F_{0}[1+V \cos (k x)],
$$

where $F_{0}=\kappa I_{0}$, and $\kappa$ is a constant. 
For our case, the monomer concentration will also be periodic and an even function of $x$. Thus, we can write $\phi(x, t), \psi(x, t)$, and $\eta(x, t)$ as Fourier series:

$$
\begin{aligned}
& \phi(x, t)=\sum_{i=0}^{\infty} \phi_{i}(t) \cos (i k x), \\
& \psi(x, t)=\sum_{i=0}^{\infty} \psi_{i}(t) \cos (i k x), \\
& \eta(x, t)=\sum_{i=0}^{\infty} \eta_{i}(t) \cos (i k x) .
\end{aligned}
$$

Substituting (18)-(20) into (13)-(15) and assuming the amplitude of the harmonics of order greater than 3 can be ignored, we obtain a set of coupled differential equations as follows:

$$
\begin{gathered}
\frac{d \phi_{0}(\xi)}{d \xi}=-\phi_{0}(\xi)-\frac{1}{2} V \phi_{1}(\xi) \\
\frac{d \phi_{1}(\xi)}{d \xi}=-V \phi_{0}(\xi)-[1+R \exp (-\alpha \xi) \cosh (\alpha V \xi)] \phi_{1}(\xi) \\
-\left[\frac{1}{2} V-R \exp (-\alpha \xi) \sinh (\alpha V \xi)\right] \phi_{2}(\xi) \\
\frac{d \phi_{2}(\xi)}{d \xi}=-\left[\frac{1}{2} V-R \exp (-\alpha \xi) \sinh (\alpha V \xi)\right] \phi_{1}(\xi) \\
-[1+4 R \exp (-\alpha \xi) \cosh (\alpha V \xi)] \phi_{2}(\xi) \\
-\left[\frac{1}{2} V-3 R \exp (-\alpha \xi) \sinh (\alpha V \xi)\right] \phi_{3}(\xi) \\
\frac{d \phi_{3}(\xi)}{d \xi}=-\left[\frac{1}{2} V-R \exp (-\alpha \xi) \sinh (\alpha V \xi)\right] \phi_{2}(\xi) \\
-[1+9 R \exp (-\alpha \xi) \sinh (\alpha V \xi)] \phi_{3}(\xi)
\end{gathered}
$$

where $R=D_{a} k^{2} / F_{0}, \xi=F_{0} t=\kappa I_{0}$. We can also treat $\psi, \eta$ with the same method. Finally, the equations are dependent on four parameters: the ratio of diffusion rate to polymerization rate, $R$, the constant that characterizes the rate of decrease of the diffusion coefficients, $\alpha$, the shrinkage rate when the monomers polymerize, $z$, and the fringe visibility, $V$. We solved the equations numerically using different parameters and got some useful results.

For our case, the ratio of monomer and liquid crystal is 7:3. However, (21)-(24) are solved numerically using MATLAB. The amplitude of the first four harmonics of monomer concentration distribution, polymer concentration distribution, and liquid crystal concentration distribution are plotted as a function of exposure $\xi$, with $R=0.5, V=1, z=0.05$, and $\alpha=0.1$. Figure 18(a) shows that all the harmonics of monomer concentration finally diminish with the time increment. This means the monomers are all polymerized. The corresponding harmonics of polymer concentration and liquid crystal concentration will saturate when the monomer are totally exhausted. Because of the shrinkage of polymer, we also can see that the polymer concentration does not

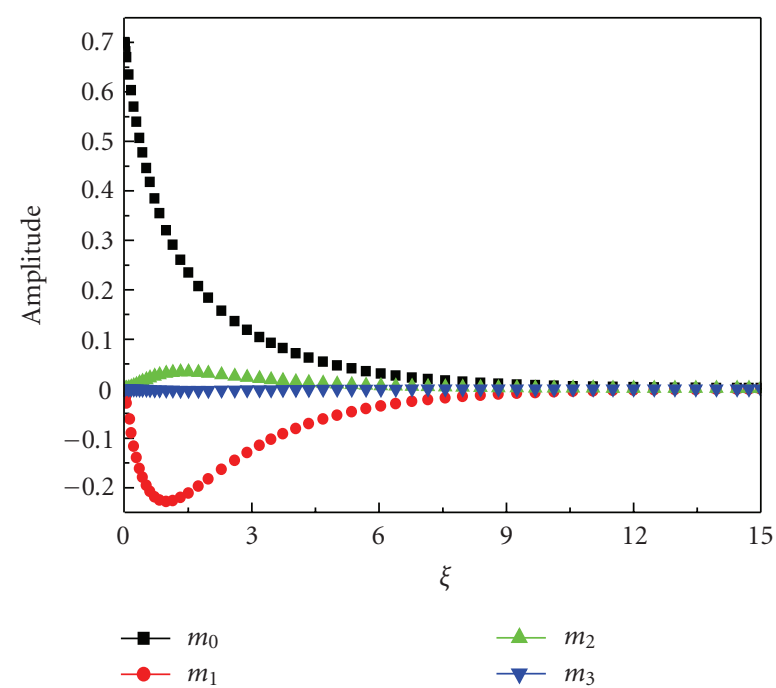

(a)

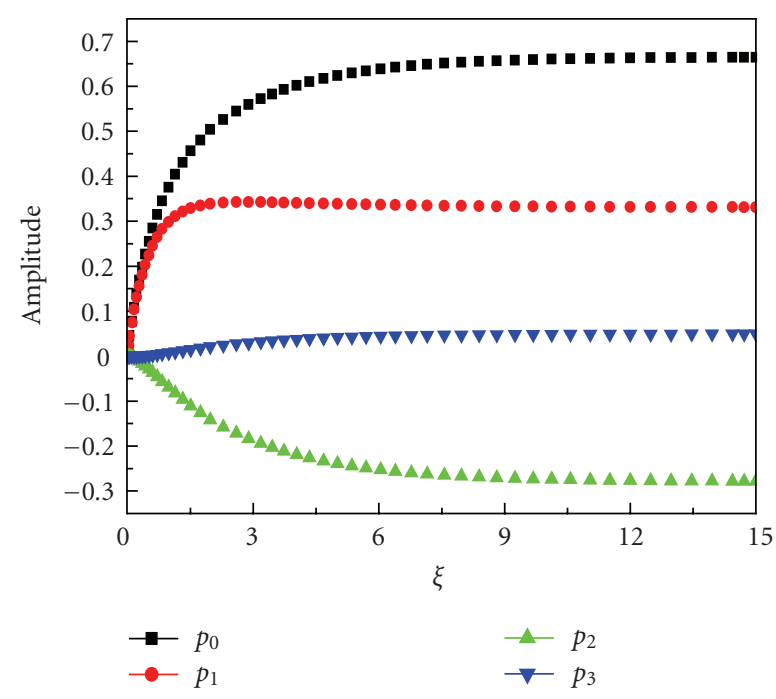

(b)

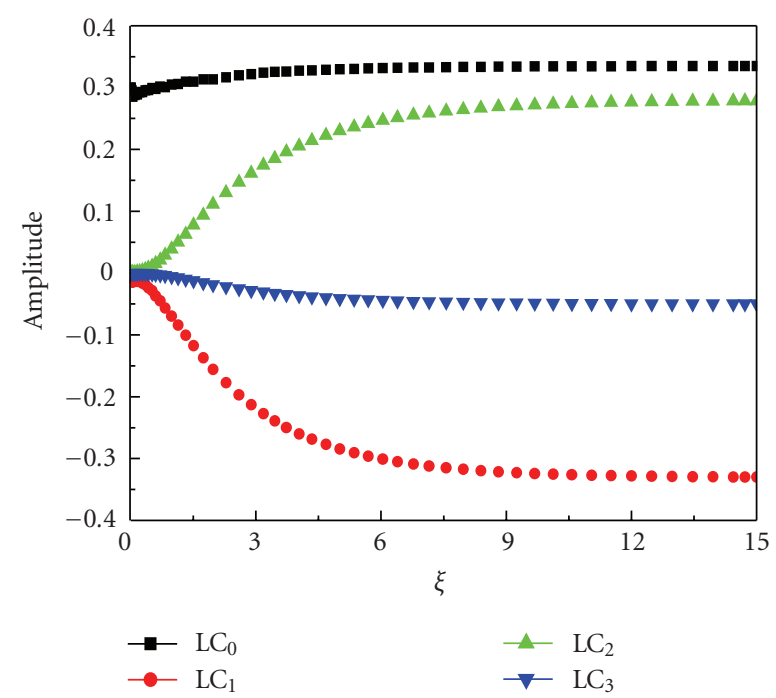

(c)

Figure 18: The harmonics of (a) monomer, (b) polymer, and (c) liquid crystal concentration as a function of time, respectively. 


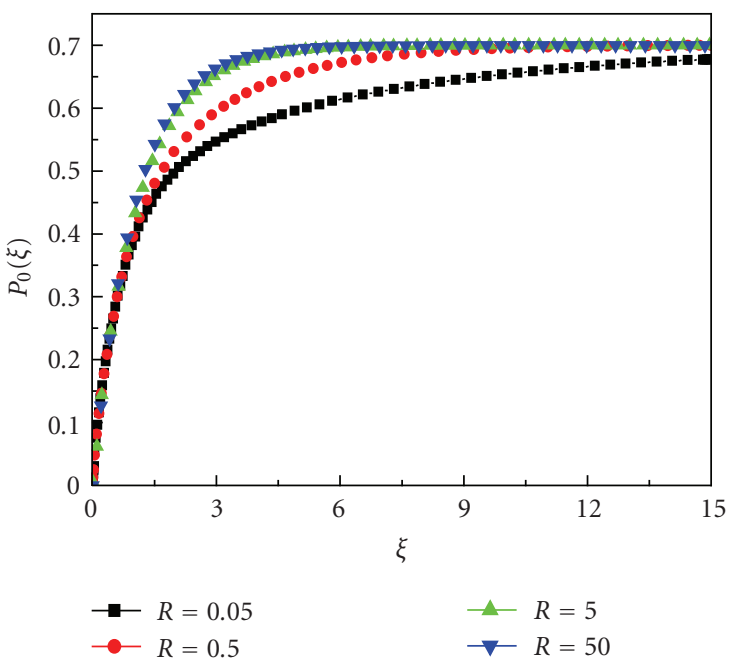

(a)

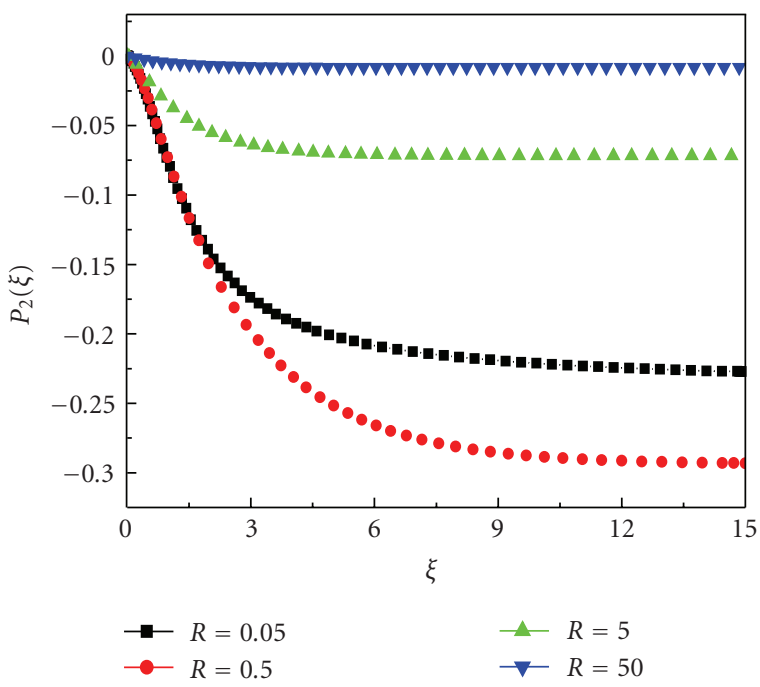

(c)

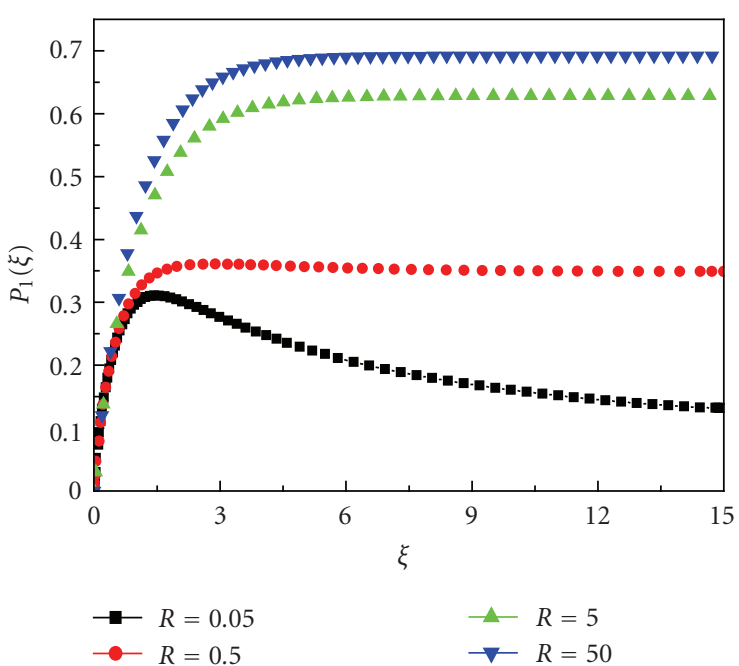

(b)

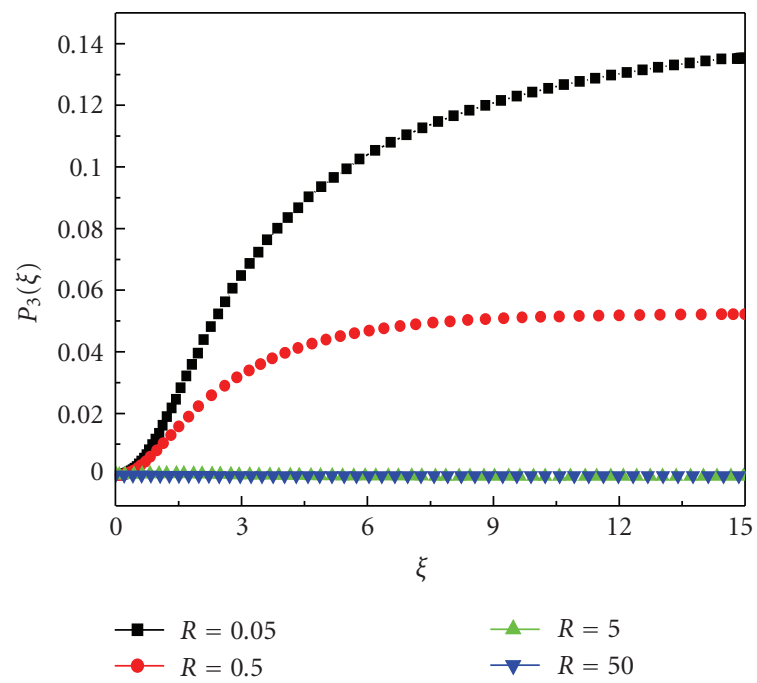

(d)

FIGURE 19: The amplitude of each harmonic of polymer concentration as a function of exposure $\xi$ for different $R$ with $V=1, z=0.05$, and $\alpha=0.1$.

reach the maximum 0.7 and the liquid crystal concentration will also have a small increment to fill with the interspace induced by the polymerization.

The ratio of diffusion rate to polymerization rate $R$ is a very important parameter that controls the formation of H-PDLC gratings. Figure 19 shows the amplitude of each harmonic of polymer concentration as a function of exposure $\xi$ for different $R$, with $V=1, z=0.05$, and $\alpha=0.1$. In general, the polymer concentration increases with the exposure and will reach a saturation value. We can see that the saturation depends strongly on $R$. Great $R$ results in large saturation values. However, for $R \gg 1$, there is almost no difference about the saturation for the zero harmonic. From Figure 19(a), we can see the zero harmonics are almost the same for $R=5$ and $R=50$. From Figure 19(c), we can see the smaller the $R$, the stronger the second harmonic. When
$R \gg 1$, the harmonics whose order more than one can be ignored because the zero and first-order harmonics dominate absolutely.

Figure 20 shows the relationship between the saturation of the first and second harmonics and the fringe visibility $V$ for different $R$. We can see that the larger the $R$, the more linear the saturation of the harmonics. For $R=50$, the saturation of the harmonics are completely linear. We also can see that the second harmonic is almost zero when $R=50$, which means no influence for the polymerization for the second harmonic.

Figure 21 shows the relationship between the saturation of the first and second harmonics and $\alpha$. We can see that $\alpha$ does not affect the grating formation strongly.

Figure 22 shows the polymer distribution profile for different $R$. The profile is purely sinusoidal for large $R$. When 


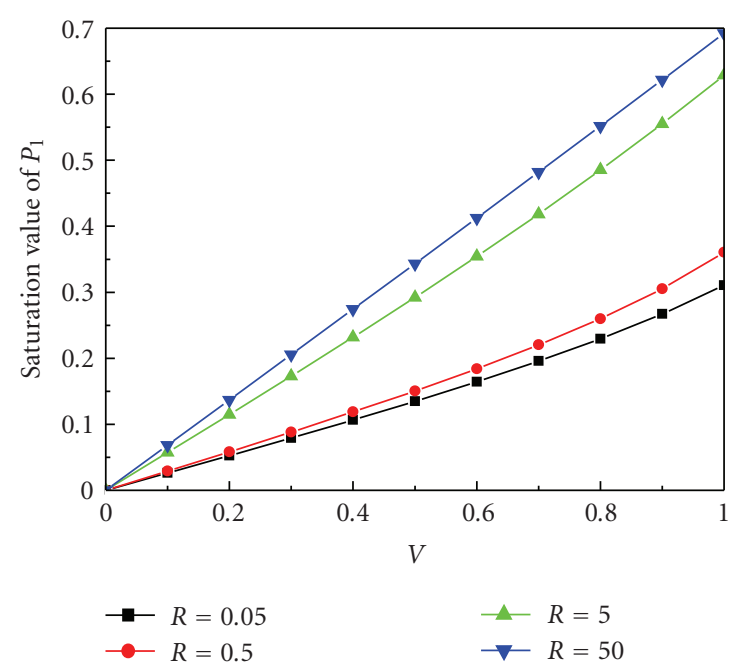

(a)

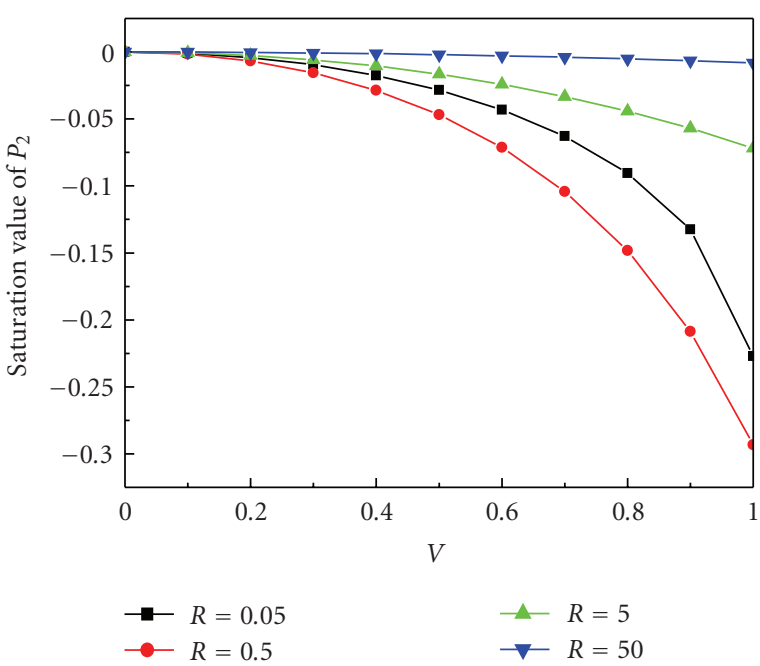

(b)

FIGURE 20: The relationship between the saturation of the first and second harmonics and the fringe visibility $V$ for different $R$.

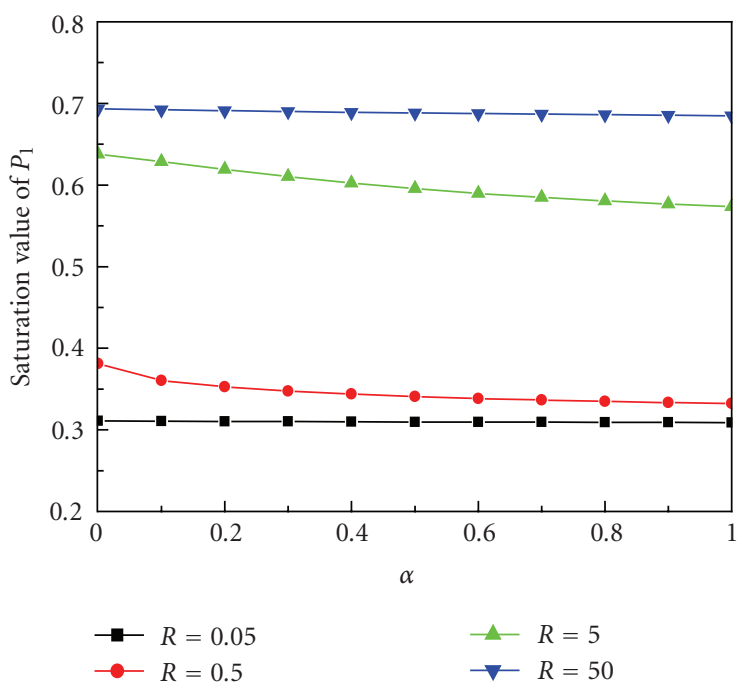

(a)

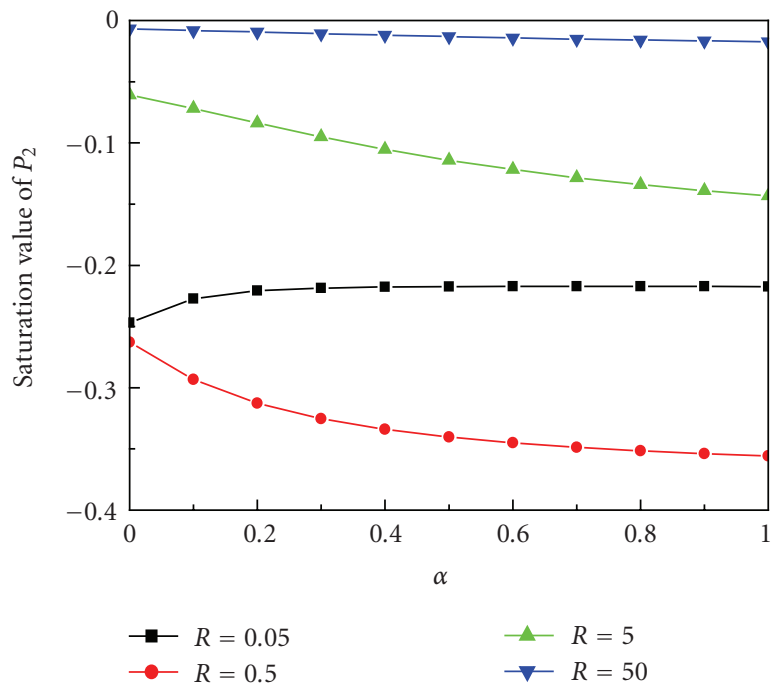

(b)

FiguRE 21: The relationship between the saturation of the first and second harmonics and $\alpha$.

the $R$ decreases, the higher harmonics will be obtained. So a large $R$ can produce a good grating structure.

Many researchers studied the H-PDLC grating formation using this simple model [58-60]. Kyu et al. gave a 2D simulation using time-dependent Ginzburg-Landau equations (TDGL model C) [61].

\section{Coupled-Wave Theory}

\subsection{Theory}

Different theories can be usaphic gratings [62, 63]. Coupledwave theory is widely used to describe thick, isotropic gratings [64]. However, an extension of the theory to optically anisotropic materials is needed when dealing with samples showing a polarization dependent behavior [6569]. Sutherland et al. gave a detailed discussion about the diffraction efficiency of H-PDLCs using coupled-wave theory [70]. The basic interaction geometry of the incident light with the holographic film, LC droplets, and applied field is shown in Figure 23. The grating is described by grating vector $\mathbf{K}$, which is perpendicular to the grating planes. Generally, the grating vector makes an angle $\varphi$ with respect to the film normal. Light is incident on the film at an angle $\theta$, as measured in the medium. For simplicity, we assume that the holographic film is sandwiched between index matching windows. Light polarized perpendicular to the plane of incidence (defined by the incident wave vector and the film normal, which are coplanar) is said to be $s$ polarized. Light polarized in the plane of incidence is said 


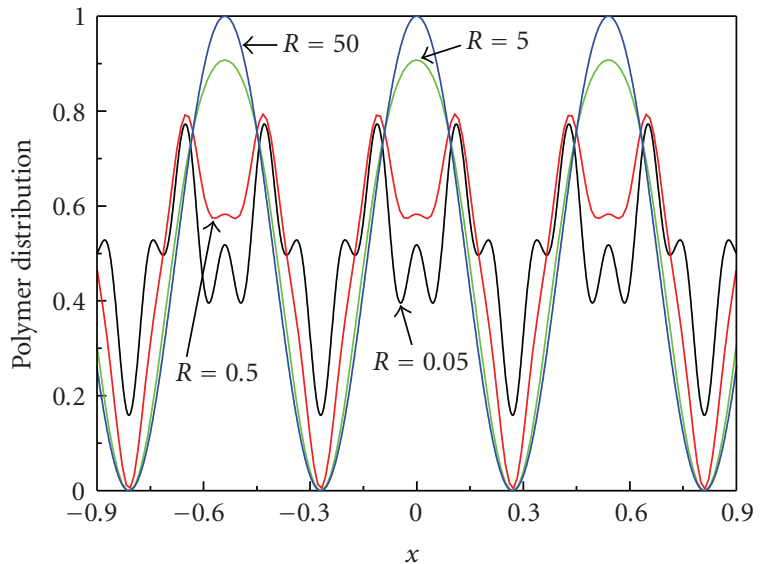

FIGURE 22: The polymer distribution profile for different $R$.

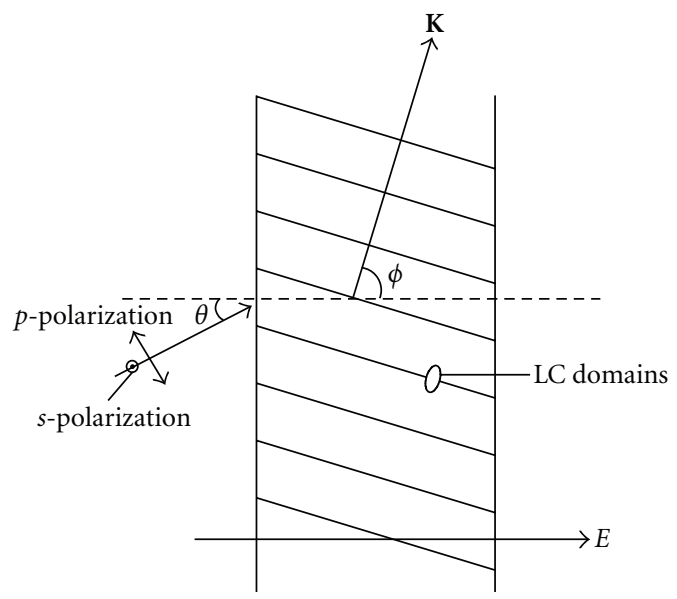

Figure 23: Geometry of H-PDLC grating.

to be $p$-polarized. Each LC droplet is taken to be a uniaxial domain, with its axis of symmetry along the applied electric field. The applied electric field is normal to the film. Here, we defined a vector $\mathbf{N}$ as the droplet director. In the absence of an electric field, $\mathbf{N}$ preferentially coincides with the dropletsymmetry axis, which is also the major axis of the elongated droplet.

Considering the pattern formed by the interference of two plane waves. The spatial distribution of irradiance is

$$
I(\vec{r})=I_{0}[1+V \cos (\vec{K} \cdot \vec{r})],
$$

where $I_{0}$ is the average irradiance, $V$ is the fringe visibility, $|\vec{K}|=2 \pi / \Lambda$, and $\Lambda$ is the fringe spacing.

The refraction index distribution generally follows the pattern of the interfering laser beams, so the expression for the index of refraction can be written as

$$
n(\vec{r})=n_{0}+n_{1} \cos (\vec{K} \cdot \vec{r}),
$$

where $n_{0}$ is the matrix index, $n_{1}$ is the index modulation amplitude.
To apply Kogelnik's theory [64], we must operate in the regime of grating known as optically thick. The criteria for this are established with reference to two parameters, namely

$$
Q=2 \pi \frac{\lambda L}{n_{0} \Lambda^{2}},
$$

where $\lambda$ is the optical wavelength and $L$ is the physical thickness of the grating. To be in the thick grating regime, we must have $Q \gg 1$. Typical gratings used in the visible spectrum have $n_{0} \sim 1.5$ and $\Lambda \sim 0.2-1.0 \mu \mathrm{m}$. Thus, the applicable thickness for the visible spectrum is in the range $L \gg 1 \mu \mathrm{m}$.

In coupled-wave theory, the two waves that interact in the medium are called incident and diffracted waves, or reference and signal waves, respectively. They are coupled by the periodic index modulation, with a coupling strength given by

$$
v_{s}=\frac{\pi n_{1 s} L}{\lambda \sqrt{c_{R}\left|c_{I}\right|}}
$$

for $s$-polarization and

$$
v_{p}=\frac{\pi n_{1 p} L}{\lambda \sqrt{c_{R}\left|c_{I}\right|}} \cos 2(\theta-\phi)
$$

for $p$-polarization. The quantities

$$
\begin{aligned}
& c_{R}=\cos \theta, \\
& c_{I}=c_{R}-\frac{\lambda}{n_{0} \Lambda} \cos \phi
\end{aligned}
$$

are called the obliquity factors. The angle of incidence may vary slightly about the principal angle, which we designate as the Bragg angle $\theta_{B}$. At this angle of incidence, the wavelength of maximum coupling is called the Bragg wavelength $\lambda_{B}$ and is defined by

$$
\lambda_{B}=2 n_{0} \Lambda \cos \left(\phi-\theta_{B}\right) .
$$

Here, the LC droplets are assumed to be uniaxial domains, the index modulation will be different for each polarization. It will also depend on the direction of wave propagation. For a slanted grating, the reference and signal waves will, thus, see a different index modulation. In this case, the index modulation amplitude is an effective value given by

$$
n_{1 j}=\chi n_{1 j, R}+(1-\chi) n_{1 j, S}
$$

where $\chi$ is an adjustable parameter, $j$ is the polarization ( $s$ or $p$ ) and the subscript $R(S)$ signifies that the value of $n_{1 j}$ depends on the propagation direction of the reference (signal) wave.

We defined a complex detuning parameter

$$
\xi=\xi^{\prime}-i \xi^{\prime \prime},
$$

where $\xi^{\prime}$ is due to a loss mechanism and $\xi^{\prime \prime}$ is due to a dephasing caused by angular and spectral departures from the Bragg condition. We have

$$
\xi^{\prime \prime}=\frac{\pi\left(\theta_{B}-\theta\right) L}{c_{S} \Lambda} \sin (\phi-\theta)+\frac{\pi\left(\lambda-\lambda_{B}\right) L}{2 n_{0} c_{S} \Lambda^{2}} .
$$


We assume that loss is entirely due to random scattering from the LC droplets, although there may be additional scattering losses due to material imperfections across gratings, and so forth. This loss mechanism is due to the fact that each PDLC slab contains a random distribution of LC droplets, and the corresponding positions of droplets in different slabs are uncorrelated. Thus, in addition to the coherent Bragg scattering from average droplet sites, there is a background of diffused incoherent scattering due to uncorrelated droplet positions. We treat this scattering as a single-particle scattering in the Rayleigh-Gans approximation [71]. Thus, we define

$$
\xi^{\prime}=\frac{\alpha_{s c} L}{4}\left(\frac{1}{c_{R}}-\frac{1}{c_{S}}\right)
$$

where

$$
\alpha_{s c}=\pi a^{2}\left(\frac{n_{d}}{n_{0}}-1\right)^{2} \Phi(k a)
$$

is the Rayleigh-Gans scattering coefficient for a spherical particle of radius $a$, with

$$
\Phi_{s}(x)=\frac{4}{9} x^{4} \int_{0}^{\pi} G^{2}\left(\frac{2 x \sin \theta}{2}\right) \sin \theta d \theta
$$

for $s$-polarization,

$$
\Phi_{p}(x)=\frac{4}{9} x^{4} \int_{0}^{\pi} G^{2}\left(\frac{2 x \sin \theta}{2}\right) \cos ^{2} \theta \sin \theta d \theta
$$

for $p$-polarization, and

$$
G(u)=\frac{3}{u^{3}}(\sin u-u \cos u) .
$$

We further define a scattering transmission factor given by

$$
\tau_{T}=\exp \left[-\frac{\alpha_{s c} L}{2}\left(\frac{1}{c_{R}}+\frac{1}{c_{S}}\right)\right]
$$

for transmission gratings.

We now recognize that because the terms that involve scattering depend on polarization, $\tau$ and $\xi$ should carry the subscript $j(j=s, p)$ to signify the appropriate polarization, and all of the preceding equations apply, with (37) used for $s$-polarization and (38) used for $p$-polarization.

We are now in a position to give expressions for diffraction efficiency. First, consider transmission gratings. In this case, only the first-order diffracted beam is of interest, and we can write

$$
\eta_{j}=\tau_{T j} S_{j} S_{j}^{*}
$$

for the diffraction efficiency of a transmission grating, where the asterisk $(*)$ signifies complex conjugate and

$$
S_{j}=v_{j} \frac{\sin \sqrt{v_{j}^{2}-\xi_{j}^{2}}}{\sqrt{v_{j}^{2}-\xi_{j}^{2}}} .
$$

For the case when scattering loss can be ignored and the angle of incidence is the Bragg angle, (41) reduces to the familiar form for transmission gratings

$$
\eta_{j}=\sin ^{2} v_{j}
$$

\subsection{Numerical Results}

For the case of an unslanted grating, the diffraction efficiency is solved numerically using Mathcad. Figure 24 gives four typical examples of diffraction efficiency as a function of the incident angle (also called angular sensitivity plots). We can see that with the increase of the index modulation (the refractive index difference between the LC-rich lamellae and the polymer-rich lamellae), the diffraction efficiency increases. The angular dependence of H-PDLCs was detailed investigated by Vita et al. [72]. Generally, to achieve a volume Bragg grating with high diffraction efficiency, a relatively large index modulation is needed.

However, it is worth noting that, for the H-PDLC transmission grating, it may be overmodulated in some cases. That is, the diffraction efficiency first passed through a maximum (generally less than 100\%) and then decreased. An obvious feature of overmodulation in the angle sensitivity plot is the relatively large side slobes. Figure 25 showed both normal and overmodulated cases. We can see that the largest side lobe of the overmodulated grating is $\sim 23 \%$, whereas for the normal grating, the largest side lobe is $\sim 10 \%$. In addition, the angular bandwidth of overmodulated is slightly narrower than that of normal. This can be easily understood by the conservation of energy.

The film thickness, $L$, and the index modulation amplitude, $n_{1}$, are reciprocal parameters, which decide the angular properties of the H-PDLC gratings. Generally, a small index modulation and a large thickness will produce a narrow angular sensitivity. The series of plots in Figure 26 showed the trend of prediction. Therefore, we can choose the parameters (i.e., film thickness and LC) according to the practical applications. For examples, for H-PDLC displays which need large view angles, a smaller thickness should be chosen.

Actually, scattering reduces the peak diffraction efficiency, preventing it from its theoretical maximum of $100 \%$. However, the droplet sizes in H-PDLC samples are $\sim 20 \mathrm{~nm}$ on average, which are very small with comparison to the visible wavelength. So the scattering loss is relatively small, as indicated in Figure 27. In fact, larger scattering losses result from not only the phase-separated LC droplets but also some other reasons such as the index mismatch at the interfaces and imperfection of the polymer film.

Generally, the diffraction efficiency of H-PDLC gratings has polarization dependence more or less because of the anisotropy of LC. Figure 28 illustrates this effect. In general, $p$-polarization is always greater. In our experiment, we indeed observed this phenomenon. This is not favorable for some applications such as optical switching which needs to be polarization insensitive. It primarily results from the shrinkage of the polymer during the formation of gratings.

For the switchable H-PDLC gratings, due to the various material formations and fabrication conditions, the polarization and electro-optical properties can be very different. Sutherland et al., further developed a theoretical model to elucidate the angle- and polarization-dependent switching behavior observed in the experiments, which combines anisotropic coupled wave theory with an elongated 


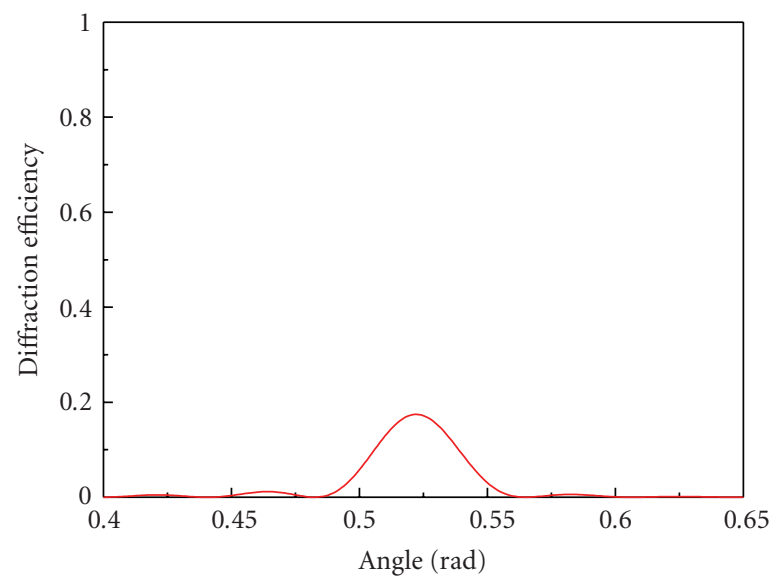

(a)

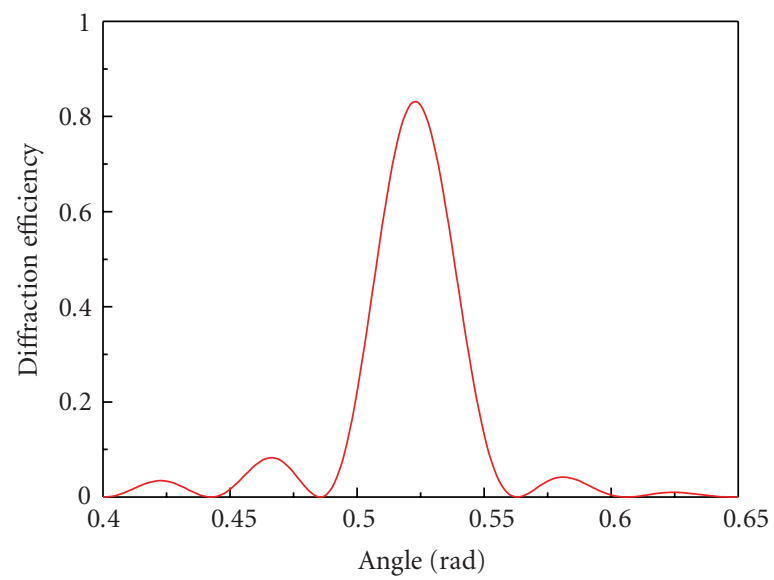

(c)

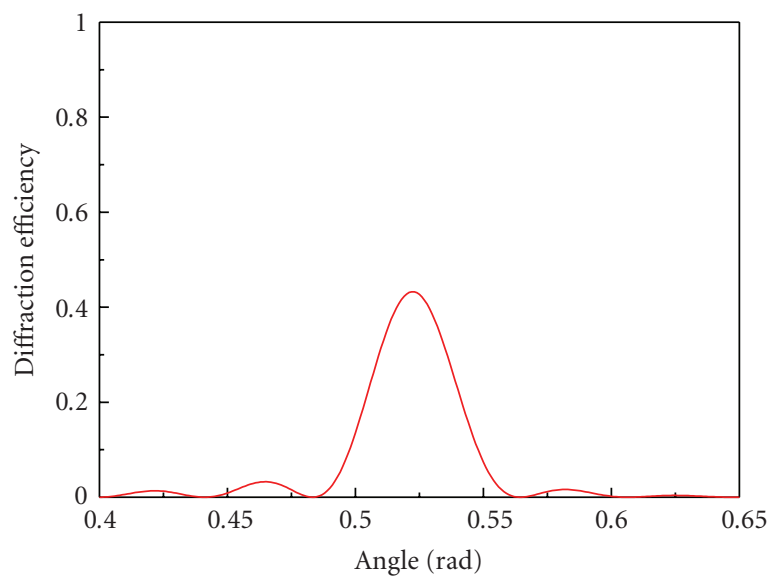

(b)

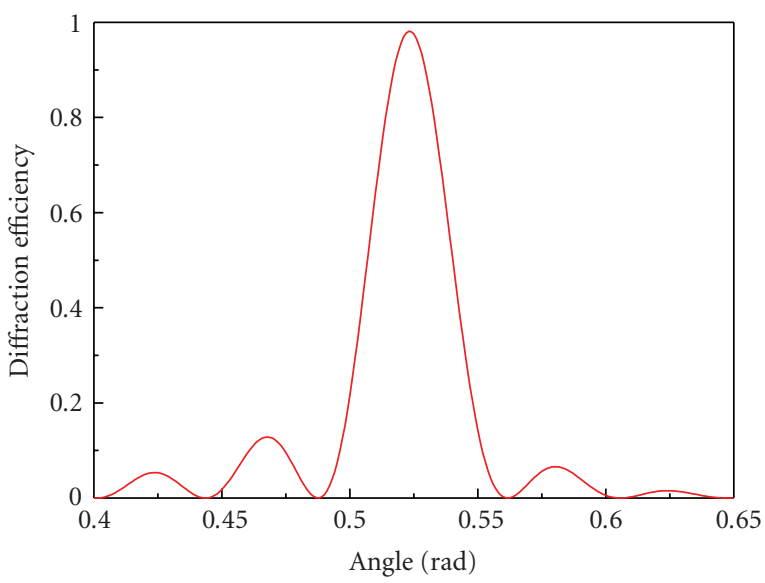

(d)

FIGURE 24: Angular dependence of diffraction efficiency for a transmission H-PDLC grating. Key parameters: $\theta_{b}=\pi / 6$ (external), $\varphi=\pi / 2$, $L=10 \mu \mathrm{m}, \lambda_{0}=633 \mathrm{~nm}, a=0.020$, (a) $n_{1}=0.015$, (b) $n_{1}=0.025$, (c) $n_{1}=0.040$, and (d) $n_{1}=0.050$.

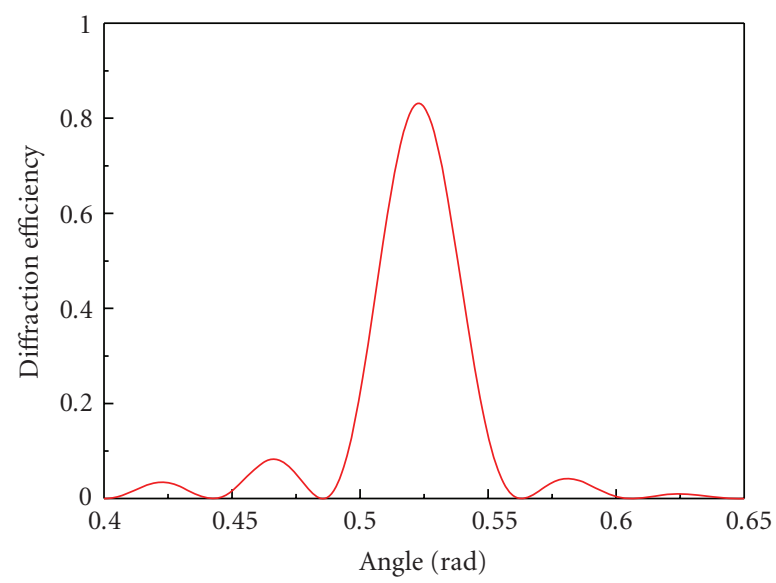

(a)

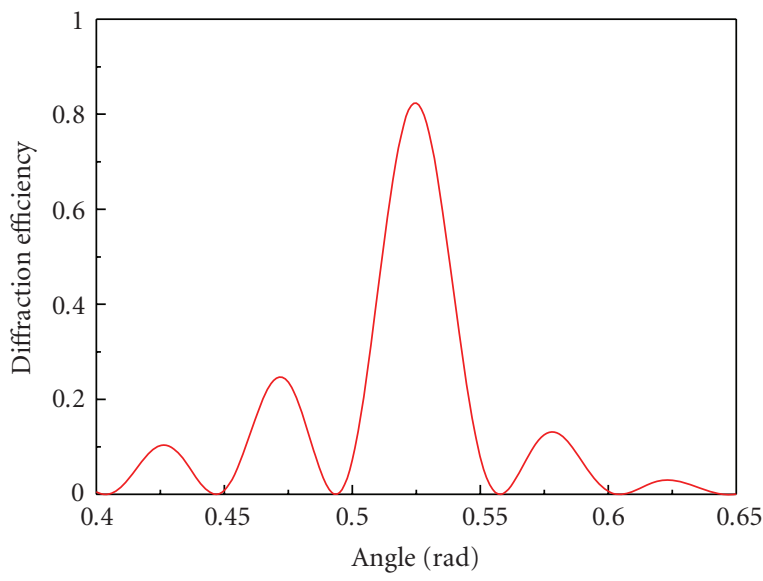

(b)

FIGURE 25: Angular dependence of diffraction efficiency for a transmission H-PDLC grating, illustrating (a) normal and (b) overmodulated cases. Key parameters: $\theta_{b}=\pi / 6$ (external), $\varphi=\pi / 2, L=10 \mu \mathrm{m}, \lambda_{0}=633 \mathrm{~nm}$, and $a=0.020$, (a) $n_{1}=0.040$, (b) $n_{1}=0.070$. 


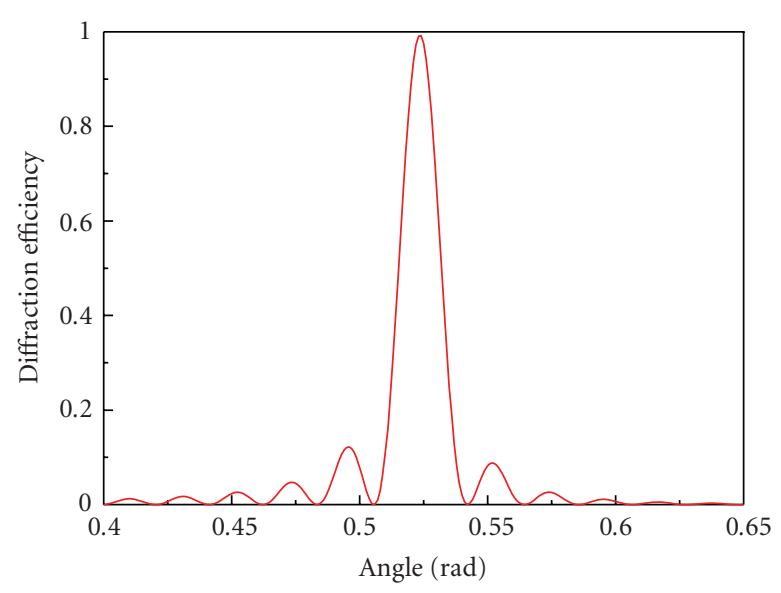

(a)

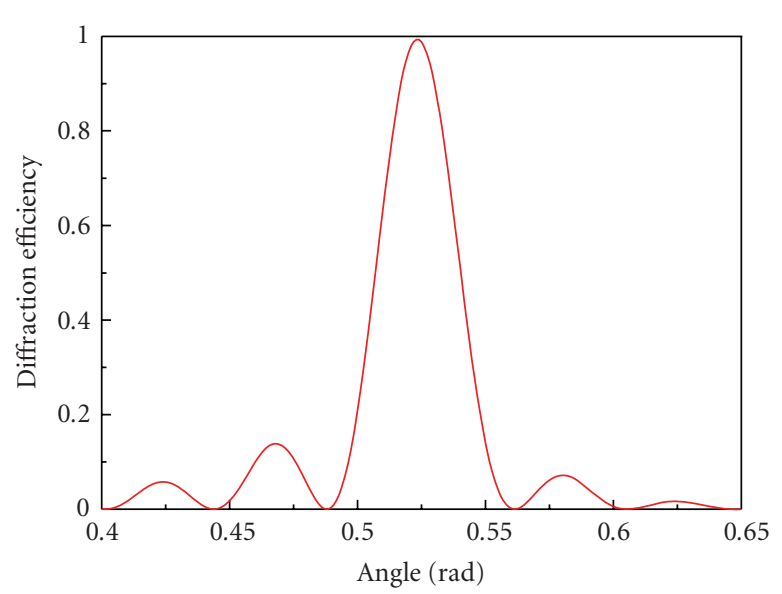

(c)

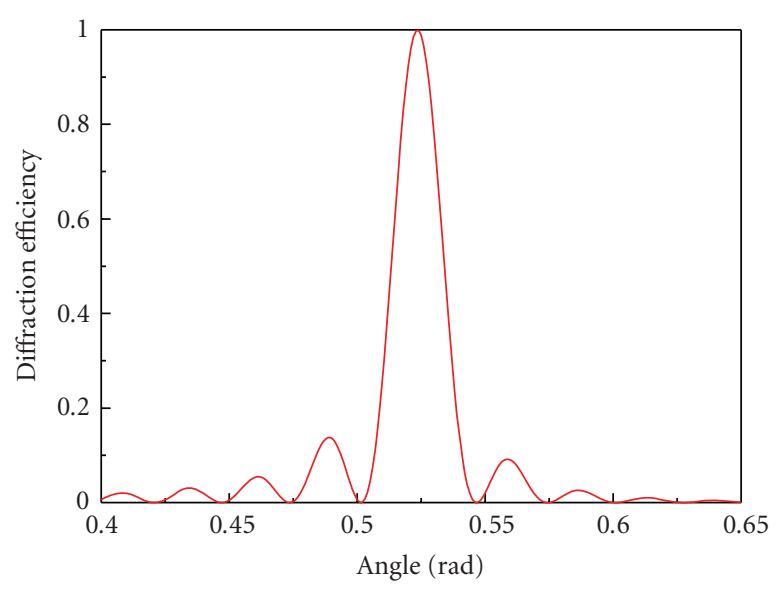

(b)

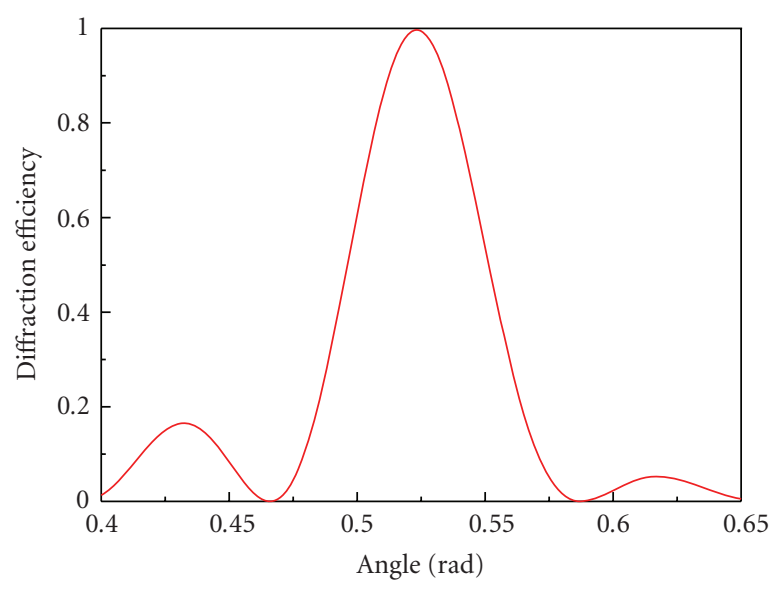

(d)

FIGURE 26: Angular dependence of diffraction efficiency for a transmission H-PDLC grating, illustrating angular bandwidth for various $L$ and $n_{1}$. Key parameters: $\theta_{b}=\pi / 6$ (external), $\varphi=\pi / 2, \lambda_{0}=633 \mathrm{~nm}, a=0.020$, (a) $L=20 \mu \mathrm{m}, n_{1}=0.026$, (b) $L=16 \mu \mathrm{m}, n_{1}=0.034,(\mathrm{c}) L=$ $10 \mu \mathrm{m}, n_{1}=0.052$, and (d) $L=6 \mu \mathrm{m}, n_{1}=0.088$.

LC droplet switching model and includes the effects of a statistical orientational distribution of droplet-symmetry axes $[73,74]$. Near infrared characterization and modeling of H-PDLCs were also done [75], which showed H-PDLCs potentially useful in telecom field.

\section{Performance Optimization}

Many researchers have explored different material recipes to achieve high diffraction efficiency, fast response time, and low drive voltage. In this article, we focused on the material recipe for visible (blue and green) curing, which is discussed in all the following parts. For this kind of material recipe, due to many different components for various purposes, many works contribute to the performance improvement of H-PDLC gratings. A clear grating morphology is desired to achieve the high performance. The morphology can be controlled by the following parts.

\subsection{Exposure Conditions}

\subsubsection{Effect of Exposure Intensity}

The exposure intensity decides the rate of gelation, diffusion, and nucleation, which determines the degree of phase separation and morphology of the droplets, and hence the diffraction efficiency. Bunning et al. speculated on the extreme morphologies possible given the relative rates of diffusion, nucleation, and gelation as shown in Figure 29 [76]. In case (a) shown in Figure 29, if the time to diffuse across $\Lambda / 2$ is fast compared to the time for droplets to nucleate and nucleation occurs much faster than gelation, a "perfect" grating is formed. If the rates of nucleation and gelation are on the same order, but diffusion of LC can still take place over a distance $\Lambda / 2$ during the polymerization and before nucleation, then little growth of the LC domains would be expected once discrete LC-rich domains are formed. These small LC domains would be spatially segregated on a 


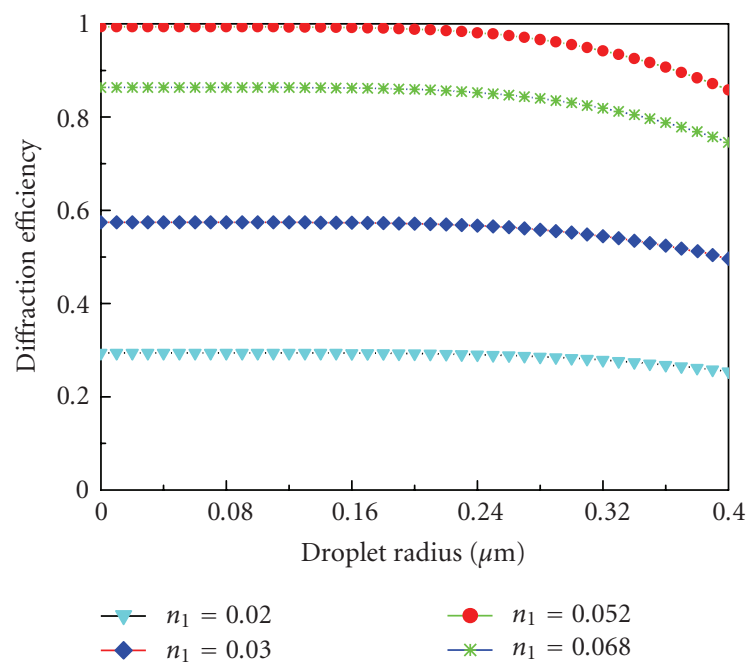

Figure 27: Peak diffraction efficiency of a transmission H-PDLC grating as a function of droplet radius $a$, illustrating the effects of scattering. Key parameters: $\theta_{b}=\pi / 6$ (external), $\varphi=\pi / 2, L=10 \mu \mathrm{m}$, and $\lambda_{0}=633 \mathrm{~nm}$.

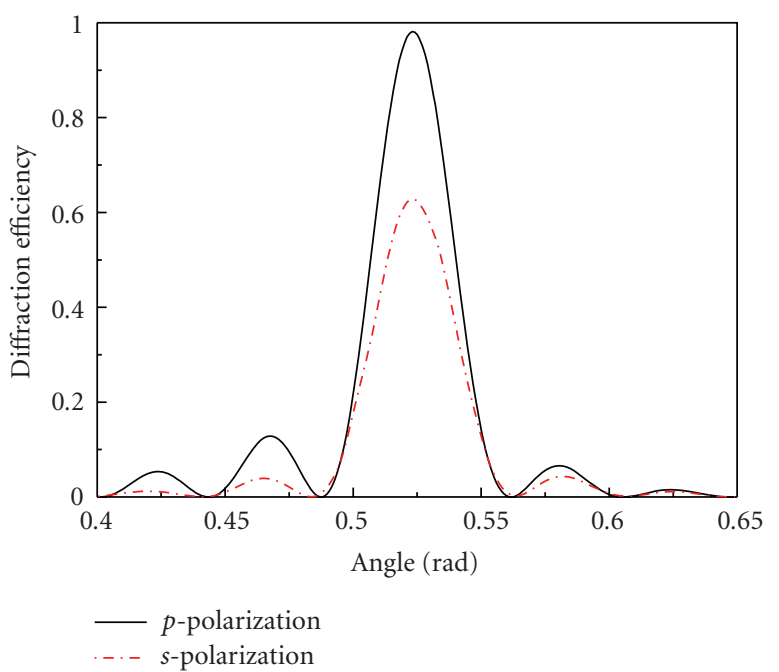

FIGURe 28: Angular dependence of a transmission H-PDLC grating, illustrating the dependence on polarization. Key parameters: $\theta_{b}=$ $\pi / 6$ (external), $\varphi=\pi / 2, L=10 \mu \mathrm{m}, \lambda_{0}=633 \mathrm{~nm}, a=0.020, n_{1}=$ 0.050 ( $p$-polarization), and $n_{1}=0.016$ ( $s$-polarization).

length scale commensurate with $\Lambda$ as shown schematically in case (b). If the LC domains separate out before the LC molecules can diffuse parallel to the grating vector a distance of $\Lambda / 2$, one would obtain cases (c) and (d) which at their extremes approximate conventional floodlit samples. One can effectively increase the time needed for diffusion of LC by increasing $\Lambda$. In the infinite case, one has a conventionally floodlit LC composite system. The size of the droplets and their distribution in sizes is again controlled by the relative rates between nucleation and gelation. If gelation is slow (case (d)), then considerable coalescence of the droplets can

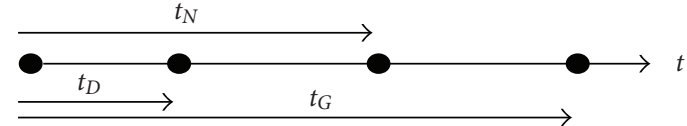

(a) $t_{D} \ll t_{N}$ and $t_{N} \ll t_{G}$

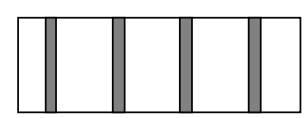

(b) $t_{D} \ll t_{N}$ and $t_{N} \approx t_{G}$

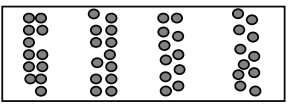

(c) $t_{D} \gg t_{N}$ and $t_{N} \approx t_{G}$

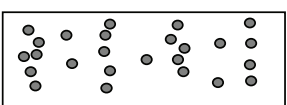

(d) $t_{D} \gg t_{N}$ and $t_{N} \ll t_{G}$

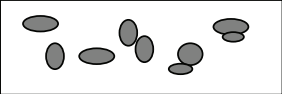

FIgURE 29: Pictorial representation of the timeline for H-PDLC grating formation. The cases (a), (b), (c), and (d) show extreme morphologies possible based on the relative time scales for the events listed on the timeline. $t_{N}, t_{G}$, and $t_{D}$ are nucleation time, gelation time, and diffusion time to $\Lambda / 2$, respectively. $\Lambda$ is the grating period [76].

be expected which increases the size distribution. If gelation is fast (case (c)) once droplets are nucleated, then small individual LC domains would be formed.

Macroscopically, if the intensity is too weak, the induced energy will not be enough, leading to a slower polymerization rate and longer LC droplets growth time. Finally, the size of LC droplets will be larger and the scattering will be increased and diffraction efficiency will be lowered. On the contrary, too strong intensity will promote the rate of polymerization. The liquid crystal droplets do not have sufficient time to grow and diffuse because of the rapid cross-linking of monomers, so many droplets were trapped in the polymerized regions. The buried droplets will act as scatterers, lowering diffraction efficiency. So exposure intensity is critical to make the rate of polymerization and diffusion approximately equal and then form a clear and smooth grating structure.

A representative plot of the data as a function of time is given in Figure 30 [11], which shows three regions characterizing the formation of holographic gratings in photopolymers: (a) a short induction period during which no polymerization takes place until all inhibitors, for example, oxygen or those added by the manufacturer to prevent accidental polymerization, have been reacted; (b) a period of rapid polymerization and photobleaching of the initiator dye with a concomitant rise in diffraction efficiency; (c) a plateau region where most of the dye molecules are irreversibly bleached. The induction period is actually quite short; measurable diffraction efficiency is noted as early as $250 \mu \mathrm{s}$ after exposure [14]. The time of rapid rise in diffraction efficiency is most certainly involved with the growth and final development of periodic LC domains. This is consistent with the final morphology revealed by SEM and by the fact that 
samples cured with no or a low concentration of LC have much lower diffraction efficiency.

According to the previous reports $[14,77,78]$, the laser intensity is varied between 0.1 and $100 \mathrm{~mW} / \mathrm{cm}^{2}$, with typical exposure time of 30-120 seconds, which strongly depends on the properties of the materials used and the component ratio in the mixture. In our experiments, we obtained the peak diffraction efficiency with the conditions of $I=10 \mathrm{~mW} / \mathrm{cm}^{2}$ and $t=120 \mathrm{~s}$ with the TMPTA monomer and prepolymer/LC $=70 / 30$.

\subsubsection{Effect of Exposure Temperature}

Another important factor to affect the structure is the exposure temperature. The temperature has a big effect on the viscosity of the materials, which is believed to affect the structure morphologies significantly. In general, at low temperatures, the diffusion rates of LCs and monomers are low due to the high viscosity of both LCs and monomers. In this case, during the exposure, some LC molecules (monomers) have not enough time to diffuse into the dark (bright) regions. As a result, many LC molecules (monomers) are trapped in the polymer-rich (LC-rich) regions, inducing an incomplete phase separation, and thus a poor grating structure is formed. At high temperatures, the LC and monomer viscosities decrease much and diffusion rates are much faster. As a result, a better separated LC and polymer structure will be formed. To obtain a complete phase separation between LC and polymer, a suitable temperature is needed. Figure 31 shows the effect of the exposure temperature on the diffraction efficiency of a transmission H-PDLC grating. At the temperature range of $40-50^{\circ} \mathrm{C}$, the diffraction efficiency reaches the maximum, which indicates the best phase separation between LCs and polymer. It is worth mentioning that at a higher temperature the prepolymer/LC mixture may polymerize rapidly so that the grating structure is not formed at all. In this case, the temperature induced phase separation (TIPS) plays a dominant role over the photopolymerization. Thus, a PDLClike structure is formed.

\subsection{Effect of Monomer Functionality}

In H-PDLC material recipe, the monomer generally has the largest concentration. Upon the light exposure, the monomer polymerizes to form a cross-linking polymer matrix. The functionality of the monomer plays a very important role in determining the degree of cross-linking in the polymer matrix, which is then anticipated to contribute to the degree of the phase separation between the polymer and liquid crystals, producing a dramatic effect on the performance of H-PDLCs. Generally, larger-functional monomer yields a highly cross-linking density, while smallerfunctional monomer yields a low or even zero crosslinking density. Fontecchio et al. investigated the monomer functionality effect on the performance of H-PDLCs by examining the switching properties and reflection efficiencies [79]. They combined monomers with functionality 2,3 , and

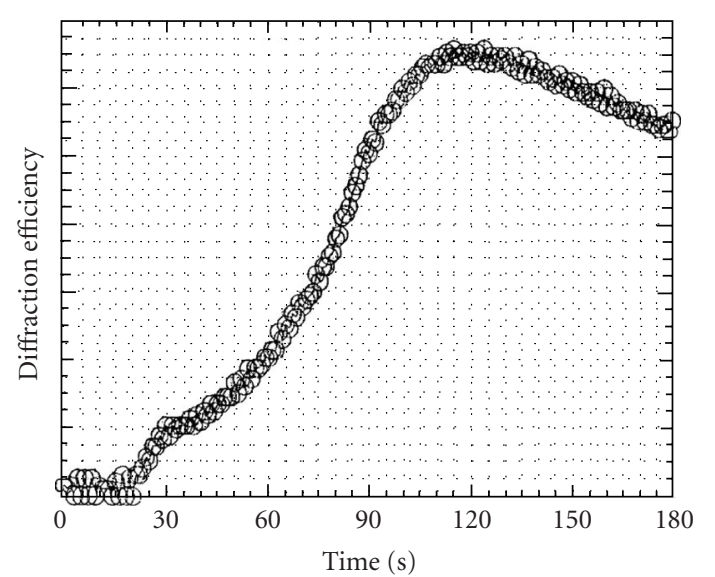

FIgURE 30: Diffraction efficiency as a function of time in an $\mathrm{H}$ PDLC grating recording [11].

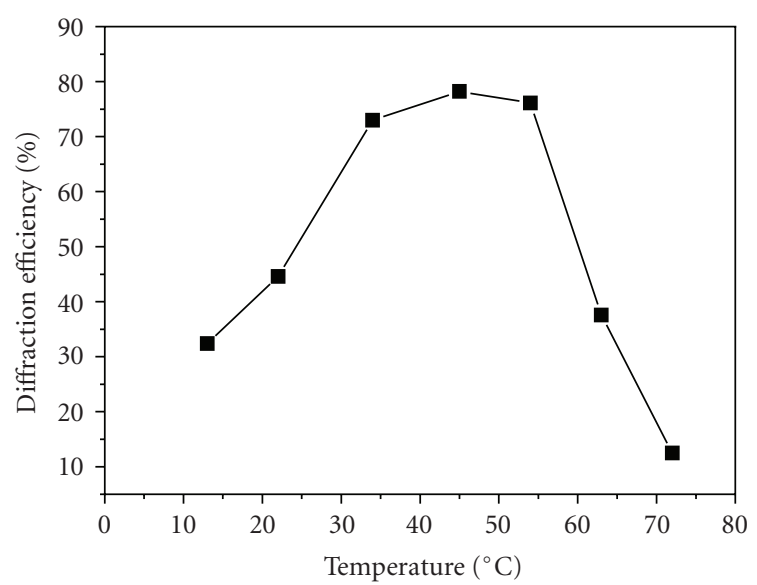

FIGURE 31: Effect of exposure temperature on the diffraction efficiency of a transmission H-PDLC grating.

6 to make integer and half-integer functionalities in the range from 2 to 6 . By measuring the reflection efficiency, switching properties, and SEM observation, the optimal monomer functionality exists for the H-PDLC polymer binder. The optimum values for the functionality to obtain the best performance are 4-4.5. Pogue et al. studied the monomer functionality effects on H-PDLCs [80], in particular, on the formation the anisotropic LC domains. The experimental results demonstrated that the local volume fraction and the LC domain size decreased substantially as the monomer functionality were decreased. The image analysis showed that a much stronger tendency to form anisotropically-shaped domains was observed for the higher functional syrups. These domain anisotropy differences are correlated with the number of reactive double bonds per monomer and are suggestive of local environmental differences exerted at the time of the domain formation.

De Sarkar and coworkers also studied the effect of monomer functionality on H-PDLCs [81]. Figure 32 shows the rate of polymerization against the monomer conversion 


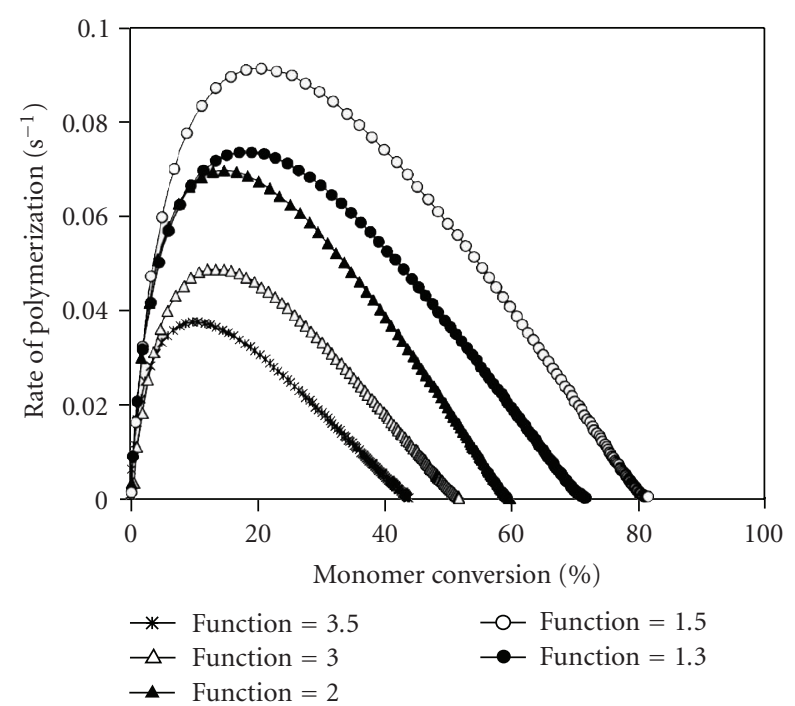

FIGURE 32: Rate of polymerization versus conversion of prepolymer solutions with different average functionalities [81].

for mixtures with different average functionalities. In general, the maximum rate of polymerization and the maximum conversion are inversely proportional to the functionality. The exception to this trend is the mixture with an average functionality of 1.3 , whose maximum rate of polymerization and extent of monomer conversion are less than the values for mixture with average functionality of 1.5 but greater than the values for average functionality of 2.0.

Figure 33 shows the effect of average functionality on the phase separated morphology of the H-PDLC gratings. The SEM observations indicate a decrease in nematic domain size in the grating morphology with decrease in average functionality of the prepolymer syrup. H-PDLC cells prepared from monomer mixtures with higher average functionality exhibit more variability in the shape and size of the LC domains. By measuring the diffraction efficiency, an increase in diffraction efficiency was observed and a decrease in LC domain size as the average functionality of the constituent monomer mixture decreases. The smaller size of LC domains can reduce the residual scattering and therefore increase the diffraction efficiency. The increase in diffraction efficiency with decrease in functionality can also be attributed to the increase in refractive index modulation. On the other hand, the switching performance of H-PDLC gratings deteriorates with decrease in functionality. The experimental results showed that an optimum average functionality $\left(F_{\text {eff }} \approx\right.$ 2.0-2.5) exists to obtain the optimized performance of $\mathrm{H}$ PDLCs below which the LC domains become negligibly small. The similar trend was also demonstrated by other researchers [82].

\subsection{Effect of LC Concentration}

LC concentration is generally the second largest concentration in the H-PDLC material recipe, which has a dramatic effect on the diffraction efficiency. In the pure polymer gratings, the peak diffraction efficiency was typically $\sim 1 \%$. With different LC concentration of $20-40 \mathrm{wt} \%$, diffraction efficiency was very different. Figure 34 showed the effect of different LC concentrations on diffraction efficiency. The peak diffraction efficiency was obtained with the concentration of $28 \mathrm{wt} \%$. The morphology of the samples was shown in Figure 35. From Figure 35, we can see that if the LC concentration is higher, the volume fraction of LC lamella throughout the bulk of the film increases and the size of droplet is larger, thus increasing the scattering and lowering the diffraction efficiency. However, if the concentration is lower, the volume fraction of LC decreases, and the size of droplets becomes smaller. Within the LCrich lamellae, a large amount of polymer exists, which lowers the index modulation and decreases the diffraction efficiency accordingly. Therefore, a suitable LC concentration is needed in order to obtain both a good grating structure and high diffraction efficiency. In most references [11, 37], the LC concentration of $\sim 30 \mathrm{wt} \%$ is the best choice to achieve high diffraction efficiency. The diffraction efficiency can reach more than $90 \%$.

\subsection{Effect of NVP Concentration}

NVP is known to be a critical component in improving acrylate-based H-PDLC grating diffraction efficiency by reducing LC droplet size $[83,84]$. As is the case in many acrylate systems, NVP causes an increase in the rate of polymerization with increasing concentration in H-PDLC formulations. This increase in the rate of polymerization occurs through the incorporation of NVP into the $\mathrm{H}$ PDLC polymer matrix. To understand the dependence of HPDLC polymerization kinetics on NVP, the influence of NVP concentration on the rate of polymerization has also been examined using photo-differential scanning calorimetry (PDSC). Figure 36(a) shows the rate of polymerization versus double bond conversion for HPDLC formulations containing increasing concentrations of NVP. For comparison, the polymerization of DPPHA with increasing NVP concentration was studied, as shown in Figure 36(b). HPDLC formulations containing up to $15 \mathrm{wt} \%$ NVP demonstrate complete NVP conversion. Examination of double bond conversion evolution in H-PDLC formulations reveals that NVP and monomer conversion are codependent at relatively low double bond conversions. This dependence results from the reaction of NVP double bonds with trapped acrylate radical species. The NVP radicals can then react with other trapped pendant acrylate double bonds. Therefore, significant rate increases and increased acrylate conversion result with NVP addition. The preferential reactivity of NVP with acrylate also serves to delay the onset of the reaction diffusion termination mechanism while also extending its predominance into higher double bond conversion.

NVP also has a major influence on LC phase separation in the formation of H-PDLCs and ultimate polymer morphology. Study of the appearance of the nematic phase, an indirect examination of phase separation, shows that samples containing NVP or the chemically similar 


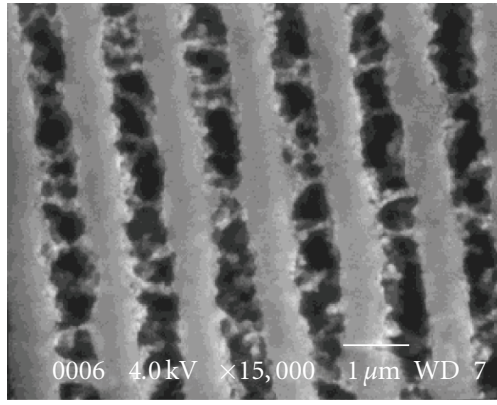

(a)

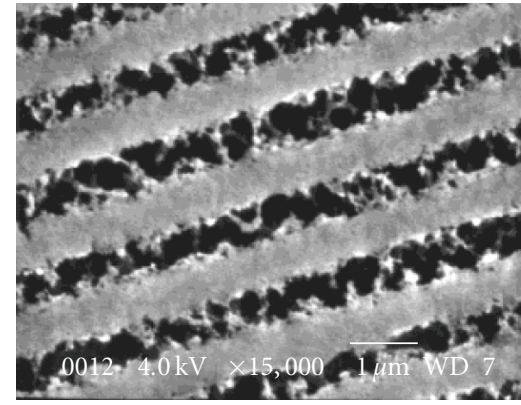

(b)

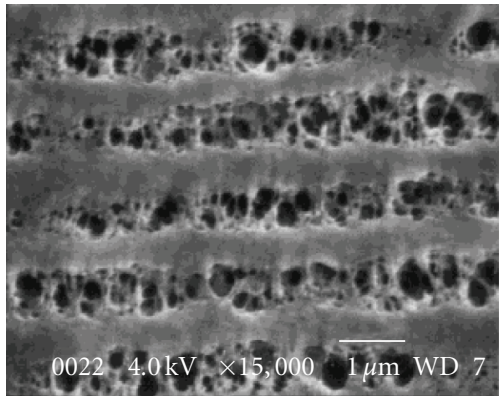

(c)

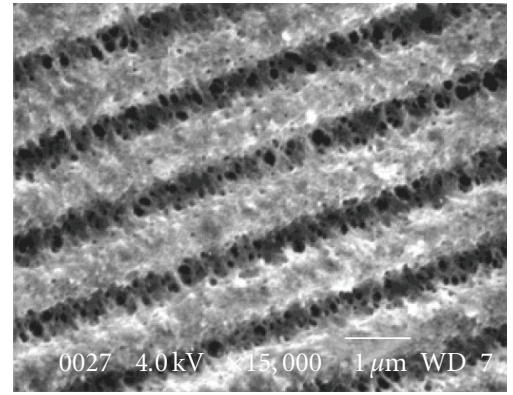

(d)

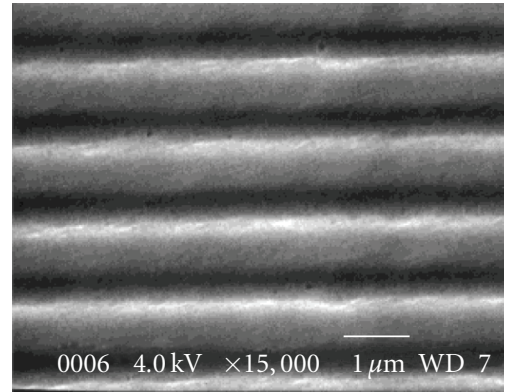

(e)

FIGURE 33: SEM micrographs for transmission gratings prepared using monomer mixtures with average functionalities of (a) 3.5, (b) 3.0, (c) 2.4 , (d) 2.0 , and (e) $1.5[81]$.

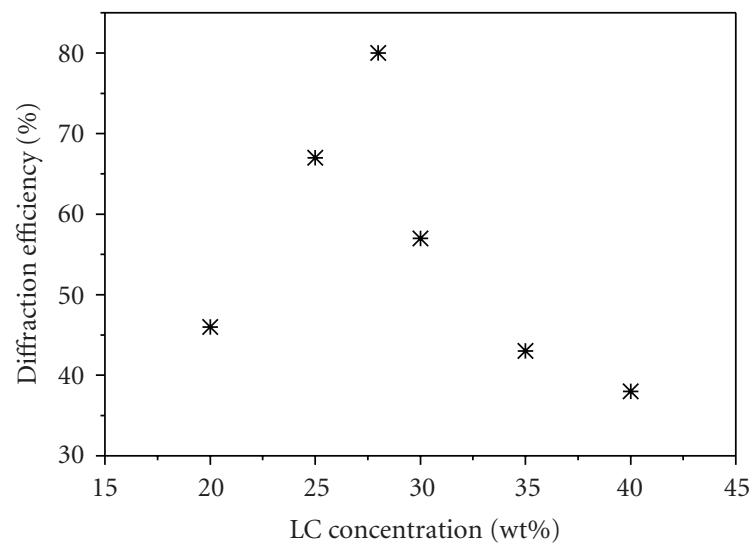

FIGURE 34: Effect of LC concentration on diffraction efficiency.

but nonreactive molecule $n$-ethyl-2-pyrrolidinone (NEP), exhibit a slower appearance of the nematic phase when compared to an H-PDLC formulation without NVP (or NEP). In general, increasing NVP concentration extends the homogeneity of the polymer/LC mixture into higher double bond conversions-slowing the LC phase separation process and the appearance of the nematic phase. Further, the kinetic influence of NVP coupled with its solubility reduces the amount of LC phase separation, which subsequently reduces the overall amount of nematic LC. These effects are also evident in the polymer/LC morphology. With NVP incorporation droplet size is much smaller than that observed when incorporating the nonreactive analogue NEP. The rate of polymerization for the H-PDLC formulation containing NVP is much greater than that containing NEP. This behavior, in turn, is the cause of the significant reduction in LC droplet size and improved H-PDLC performance with NVP inclusion. Figure 37 shows obviously different surface morphologies of H-PDLC transmission gratings prepared with $4 \mathrm{wt} \%$ and $15 \mathrm{wt} \% \mathrm{NVP}$, respectively. It can be seen that increasing the amount of NVP leads to smaller droplets and decreases the anisotropy of the LC droplets.

\subsection{Decreasing Driving Voltage}

For H-PDLC gratings, the LC droplets are small and the anchoring energy is high, which results in a high driving voltage. According to Mormile et al., the threshold voltage of PDLC can be written as [85]

$$
V_{\mathrm{th}} \approx \frac{d}{r}\left[\frac{K\left(\ell^{2}-1\right)}{\varepsilon_{0} \Delta \varepsilon}\right]^{1 / 2},
$$

where $d$ is the HPDLC film thickness, $r$ is the droplet radius, $K$ is the effective elastic constant, $\varepsilon_{0}$ is the vacuum dielectric constant, $\Delta \varepsilon$ is the LC dielectric anisotropy, $\ell=a / b$ is the droplet aspect ratio, with $a$ and $b$ the length of the major and minor axis of the ellipsoid-shaped droplet, respectively. Equation (44) was deduced by balancing the elastic and field torques to the equilibrium orientation at the presence of a voltage applied to a shaped droplet [86], that is, $\Gamma_{d}+\Gamma_{e}=0$, where $\Gamma_{d}$ is the elastic torque per unit volume and $\Gamma_{e}$ is the electric torque on the droplet director per unit volume. This 


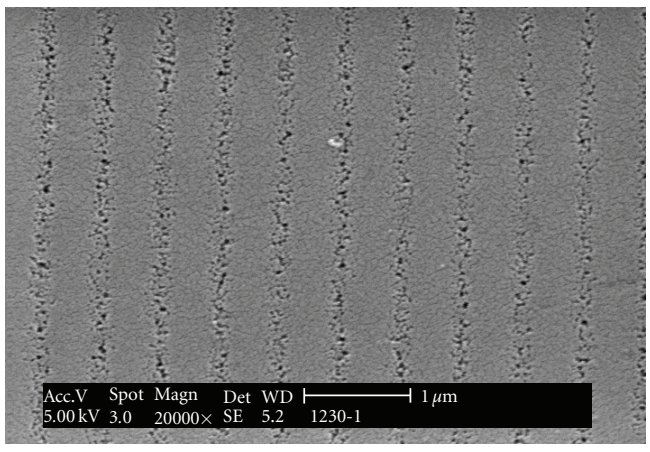

(a)

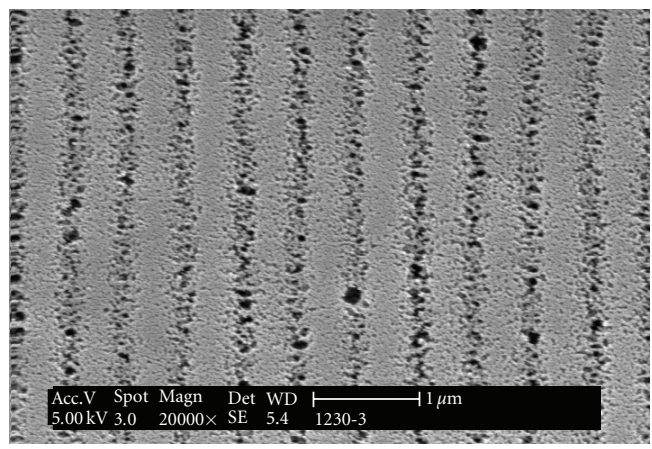

(c)

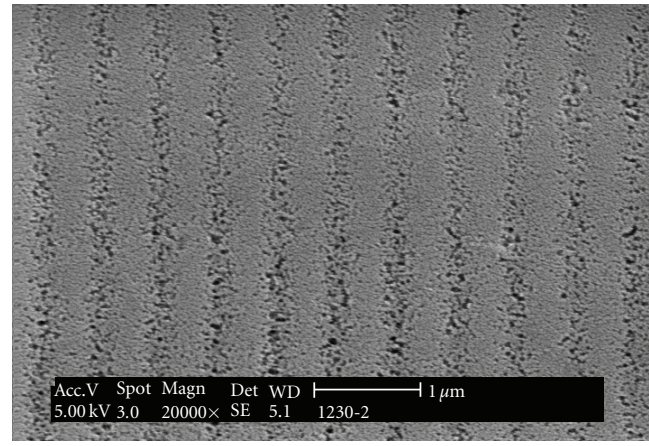

(b)

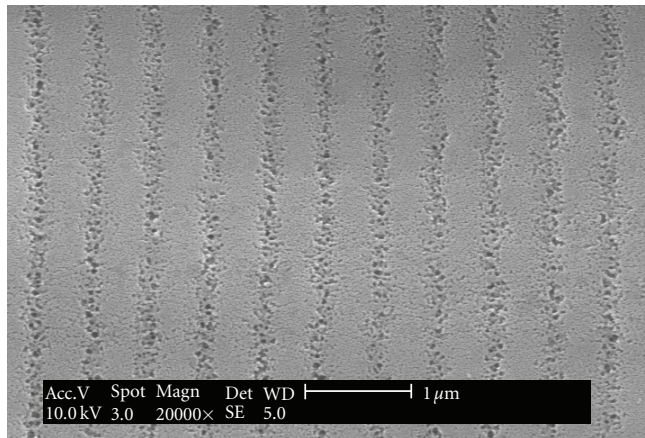

(d)

FIGURE 35: The same magnification SEM photographs of the surface of H-PDLC Bragg grating of different LC contents: (a) 20 wt\%, (b) $30 \mathrm{wt} \%$, (c) $40 \mathrm{wt} \%$, and (d) $28 \mathrm{wt} \%$.

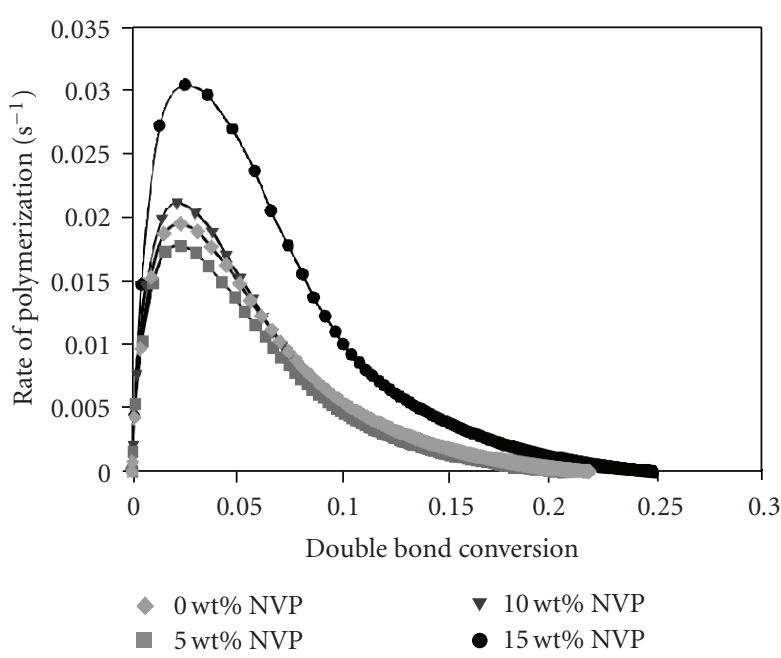

(a)

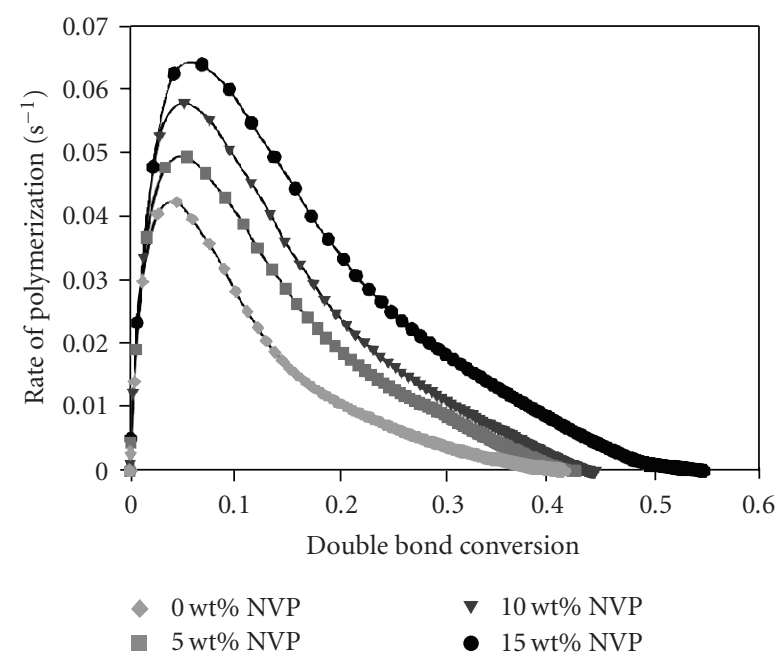

(b)

FIGURE 36: Rate of polymerization versus double bond conversion for (a) HPDLC formulations with $0(\diamond), 5(\square), 10(\nabla)$, and $15(\bullet)$ wt $\%$ NVP and (b) DPPHA:NVP formulations with $0(\diamond), 5(\square), 10(\nabla)$, and $15(\bullet)$ wt $\%$ NVP

can be regarded as the shape contribution to the threshold voltage of a normal PDLC.

The LC droplets of conventional PDLC are about $1 \sim 5 \mu \mathrm{m}$ in size. However, the size of HPDLC droplets is about $0.01 \sim 0.1 \mu \mathrm{m}$. The size effect on the threshold voltage can be understood by the following comparisons. Using our experimental parameters, the cell thickness, $d$, is $20 \mu \mathrm{m}$, the elastic constant, $K$, is $1.71 \times 10^{-11} \mathrm{~N}$, the dielectric anisotropy, $\Delta \varepsilon$, is 6.49 , and assuming an aspect ratio of 1.1, for LC droplets with $r=2 \mu \mathrm{m}$ and $0.02 \mu \mathrm{m}$, the 


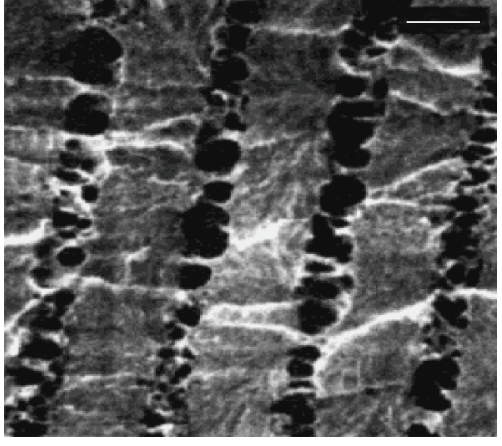

(a)

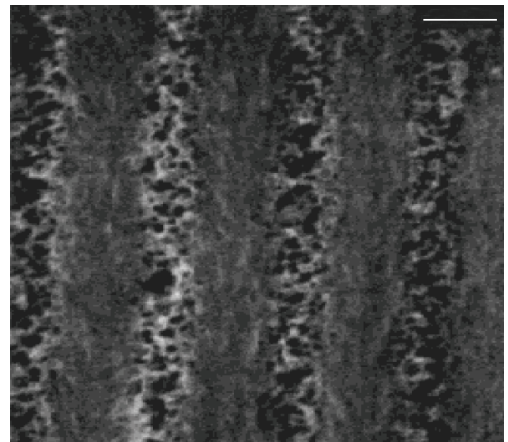

(b)

Figure 37: SEM images of H-PDLC transmission gratings with (a) $4 \mathrm{wt} \%$ and (b) $15 \mathrm{wt} \%$ NVP. Scale bars represent $600 \mathrm{~nm}$ [83].

corresponding threshold voltage $V_{\text {th }}$ calculated is $2.5 \mathrm{~V}$ and $250 \mathrm{~V}$, respectively. It can be seen that with the reduction in dimension, the threshold voltage increases linearly. In general, the threshold voltage of nano-PDLC produced by holography is about one or two orders higher than that of normal PDLC.

For practical application of HPDLC, it is desired to reduce the driving voltage. Colegrove et al. reported that by adding a high dielectric anisotropy material $(\Delta \varepsilon=65)$, the threshold voltage of HPDLC can be reduced significantly [87]. In addition, adding a small portion of surfactant to the prepolymer/LC syrup also helps to reduce the threshold voltage [88-91]. Fluorination of the polymer matrix also has significant effects on decreasing the driving voltage of $\mathrm{H}$ PDLC $[92,93]$. In the following, the effects of surfactants and polymer fluorination will be discussed.

\subsubsection{Surfactant Effect}

The role of surfactant is supposed to be a lubricant between polymer matrix and liquid crystals. When phase separation occurs in the homogenous mixture, the surfactant will form an intermediate layer between the polymer and the LC. The anchoring energy at the interface of the LC and the surfactant becomes weaker than that at the interface of the LC and polymer without surfactant. As a result, the liquid crystal molecules could be easily reorientated under an electric field. Therefore, the decrease in anchoring strength may effectively lower the driving voltage.

Figure 38 shows the diffraction efficiency as a function of driving electric field for three different surfactants. The H-PDLC without surfactant is also shown for comparison. It can be seen from Figure 38 that, the surfactant is effective in reducing the driving voltage. The conductivity of samples with surfactants number 1 and number 3 was so high that the voltage could not be increased further before they were completely switched. Sample number 2 can both keep the high diffraction efficiency and reduce the driving voltage effectively. It reduces the threshold voltage from $13 \mathrm{~V} / \mu \mathrm{m}$ to about $2.5 \mathrm{~V} / \mu \mathrm{m}$.

The surfactant also has a significant effect on the morphologies of H-PDLC gratings. Figures 39(a) and 39(b) show the SEM morphologies of the HPDLC gratings, which

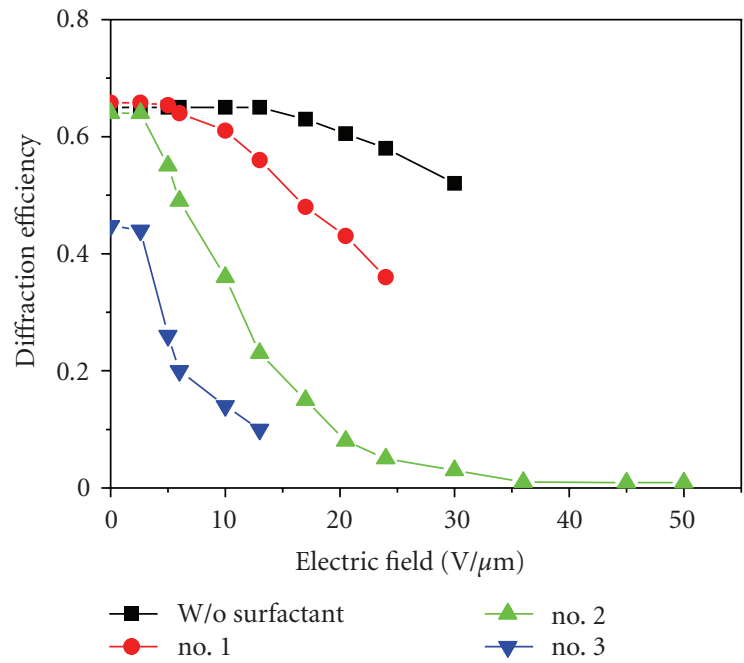

Figure 38: Diffraction efficiency versus the electric field curves for Bragg gratings made of liquid crystal E7 with three different kinds of surfactants, octanoic acid (no. 1), S-271 POE sorbitan monooleate (no. 2), and Tergital Min-Foam $1 \mathrm{X}$ (no. 3), respectively. The diffraction efficiency without surfactant is also shown for comparison.

contain $8 \mathrm{wt} \%$ and $15 \mathrm{wt} \%$ of surfactant, respectively. From Figure 39, it can be seen that the surfactant affects the grating significantly. With more surfactant, the polymer regions contain more LC droplets. Thus the surfactant affects the LC movement during polymerization. Figure 39(a) has a clearer grating structure than Figure 39(b), which indicates that the grating in Figure 39(a) has better diffraction properties. In our experiment, when the surfactant content was around $8 \mathrm{wt} \%$, the HPDLC Bragg gratings could both keep high diffraction efficiency and have excellent electro-optical properties.

Figure 40 shows the diffraction efficiency as a function of the surfactant content for samples containing number 2 surfactant. With the increase of the content, the diffraction efficiency reduces. Generally, when the surfactant content was less than $8 \mathrm{wt} \%$, the HPDLC Bragg gratings could still keep higher diffraction efficiency. 


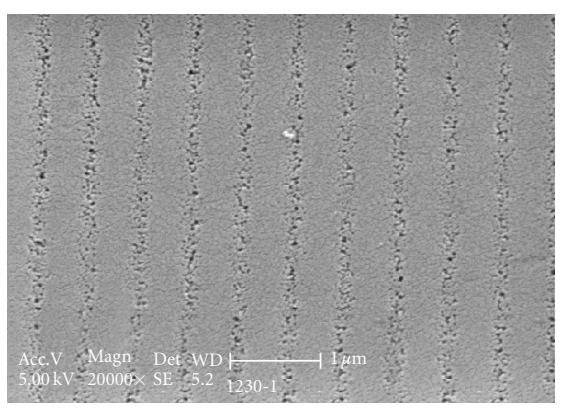

(a)

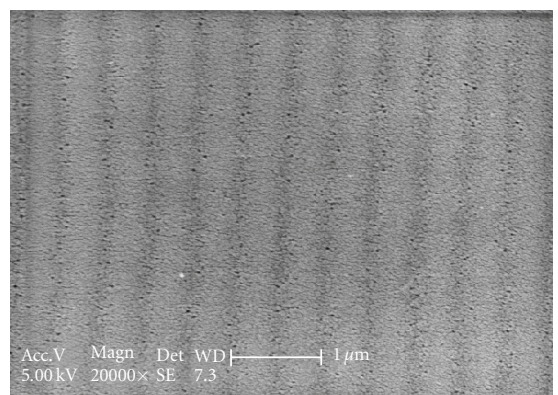

(b)

Figure 39: SEM images of the HPDLC Bragg gratings consisting $8 \mathrm{wt} \%$ (a) and $15 \mathrm{wt} \%$ (b) of surfactant no. 2. The monomer and liquid crystal used are no. 3 in Figure 12 and E7, respectively.

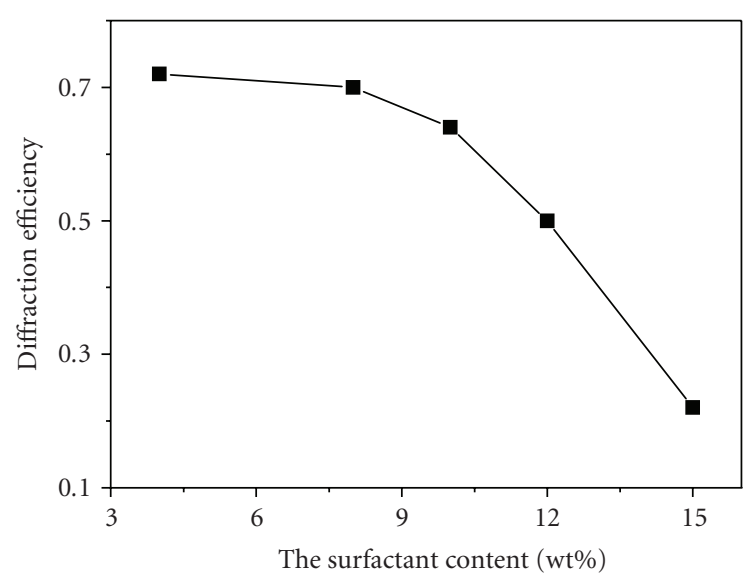

Figure 40: Diffraction efficiency as a function of the surfactant content for surfactant no. 2. The monomer and liquid crystal used are no. 3 in Figure 12 and E7, respectively.

\subsubsection{Fluorination Effect}

Another way to reduce the driving voltage is to modify the monomers. The experimental results have shown the partial fluorination of the polymer matrix has significant effects on the morphology and electro-optical performance of $\mathrm{H}$ PDLC $[92,93]$. The morphology of the fluorinated transmission gratings displayed an enhanced phase separation as manifested in a significant increase in nematic domain size and the LC volume fraction, as shown in Figures 41 and 42. The incorporation of fluorinated monomers in the standard UV H-PDLC formulation reduces the switching voltage considerably. However, the tradeoff is that the relaxation time also increases with fluorination. The experimental results strongly indicate that significant amount of fluorine atoms resides at the polymer-LC interface. The presence of fluorine atoms at the interface can manipulate the surface anchoring strength and alignment, causing a reorientation of the original configuration of the LC droplet directors.

From the above experimental results, both surfactant and fluorination has a significant effect in decreasing the driving voltage. However, there are side-effects. For example, the grating morphology was changed and the diffraction efficiency was decreased more or less with addition of surfactants. The polymer fluorination seems better to decrease the driving voltage compared to the surfactant, but the longer relaxation time is not desired.

\subsection{Index Matching Effect}

The index matching between the polymer matrix and liquid crystal plays an important role in the performance optimization of H-PDLCs. In our configuration, the polymer refractive index, $n_{\mathrm{p}}$, is chosen to be as close as possible to the ordinary refractive index, $n_{0}$, of LC. This helps to reduce the diffraction loss in the transparent state. Moreover, the birefringence of LC, $\Delta n$, is chosen to be as large as possible in order to improve the diffraction efficiency. We experimentally optimized the material recipe according to the refractive index match. In our experiment, five kinds of acrylate monomers were selected and their refractive indices are 1.490 for DPPHA (no. 1), 1.484 for PETA (no. 2), 1.474 for TMPTA (no. 3), 1.487 for PET(etra)A (no. 4) and 1.450 for TPGDA (no. 5), respectively. The chemical structures of these five monomers are shown in Figure 12 and their corresponding functionalities are 5-6, 3, 3, 4, and 2 , respectively. Because the prepolymer syrup includes other chemicals such as photoinitiator and coinitiator, moreover, the refractive index changes during polymerization. In general, the refractive index will be different from the above values for the mixture after polymerization. Two different LCs, E7, and BL038, were chosen to match the refractive index of polymer matrices and their physical properties were listed in Table 1.

The refractive indices of the monomers are from Sigma-Aldrich. Because other chemicals are mixed into the monomer, the refractive index of the prepolymer syrup changes accordingly. The prepolymer syrup refractive indices were measured by Abbe refractometer. Then the syrup was cured on a $\mathrm{SiO}_{2}$ substrate to form a polymer thin film, the refractive index, $n_{\mathrm{p}}$, of which was measured by m-line method [94]. Figure 43 shows the setup of refractive index 


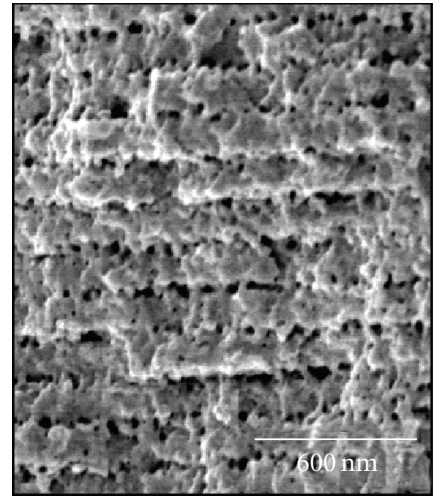

(a)

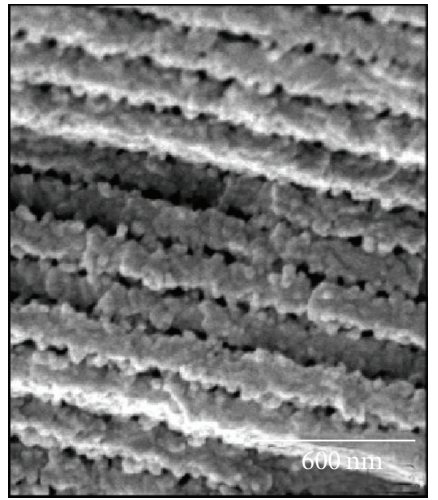

(b)

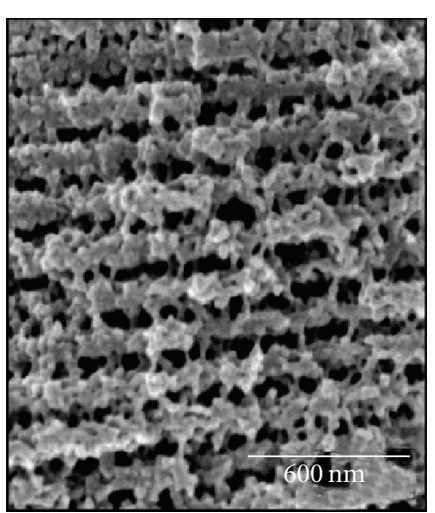

(c)

FIGURE 41: LVHRSEM photomicrographs of (a) standard formulation, (b) 13.2 mol\% MA, and (c) 11.7 mol\% HFIPA holographic PDLC films [92].

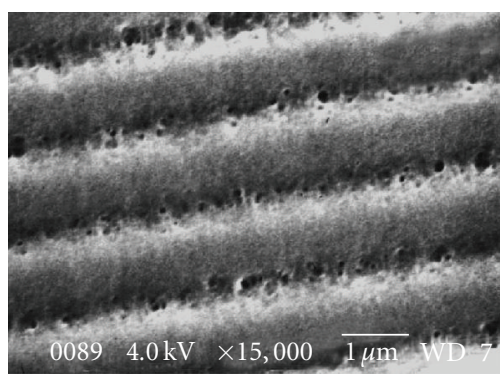

(a)

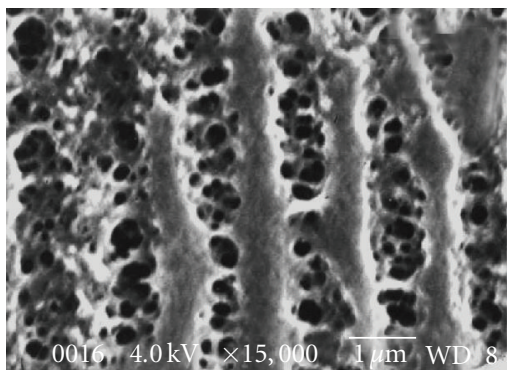

(d)

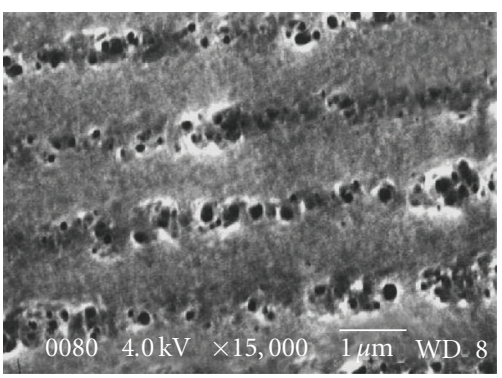

(b)

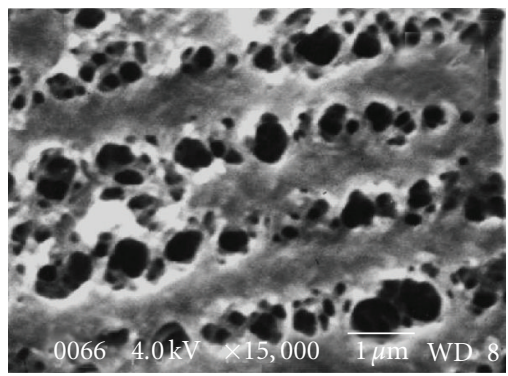

(e)

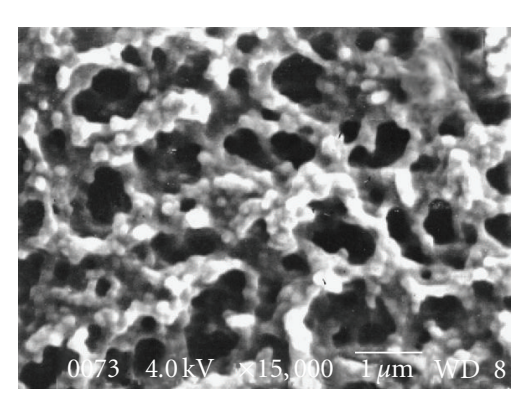

(g)

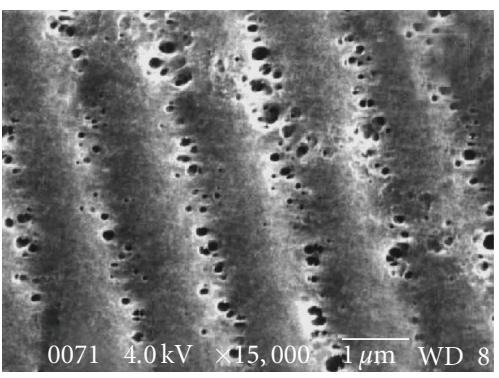

(c)

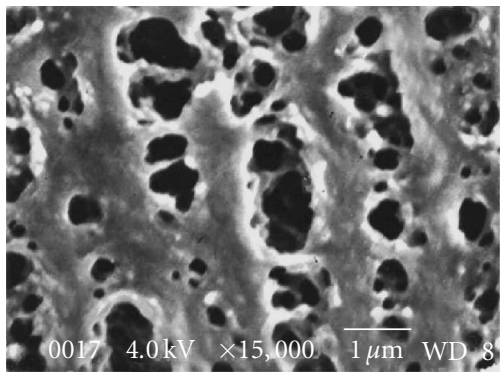

(f)

FIGURE 42: SEM micrographs of HPDLC transmission gratings prepared (a) from control monomer mixture and substituting it with, (b) $10 \mathrm{wt} \%$, (c) $20 \mathrm{wt} \%$, (d) $25 \mathrm{wt} \%$, (e) $30 \mathrm{wt} \%$, (f) $35 \mathrm{wt} \%$, and (g) $40 \mathrm{wt} \%$ of HFIPA [93]. 
TABLE 1: Basic physical properties of LC E7 and BL038.

\begin{tabular}{lccc}
\hline Physical properties & & E7 & BL038 \\
\hline Clearing point $\left({ }^{\circ} \mathrm{C}\right)$ & & +58.0 & +100.0 \\
& $20^{\circ} \mathrm{C}$ & 39 & 72 \\
Flow viscosity $\left(\mathrm{mm}^{2} \mathrm{~s}^{-1}\right)$ & $0^{\circ} \mathrm{C}$ & 145 & - \\
& $-20^{\circ} \mathrm{C}$ & 1200 & - \\
& $-30^{\circ} \mathrm{C}$ & 6400 & - \\
Optical anisotropy $+20^{\circ} \mathrm{C}, 589.3 \mathrm{~nm}$ & $\Delta n$ & 0.2253 & 0.2720 \\
& $n_{e}$ & 1.7464 & 1.7990 \\
& $n_{\mathrm{o}}$ & 1.5211 & 1.5270 \\
Dielectric anisotropy $+20^{\circ} \mathrm{C}, 1.0 \mathrm{kHz}$ & $\Delta \varepsilon$ & +13.8 & +16.9 \\
& $\varepsilon_{\perp}$ & 5.2 & 6.1 \\
& $\varepsilon_{\|}$ & 19.0 & 23.0 \\
\hline
\end{tabular}

TABLE 2: Refractive index and corresponding diffraction efficiency for various monomers used.

\begin{tabular}{lccccc}
\hline No. & 1 & 2 & 3 & 4 & 5 \\
\hline Monomer & 1.490 & 1.484 & 1.474 & 1.487 & 1.450 \\
Prepolymer syrup & 1.501 & 1.496 & 1.489 & 1.498 & 1.471 \\
Cured film & 1.529 & 1.528 & 1.522 & 1.530 & 1.502 \\
DE for E7 & $41.2 \%$ & $56.4 \%$ & $65.0 \%$ & $41.6 \%$ & $32.2 \%$ \\
DE for BL038 & $61.8 \%$ & $68.4 \%$ & $40.2 \%$ & $51.6 \%$ & $34.2 \%$ \\
\hline
\end{tabular}

measurement using $\mathrm{m}$-line method. The results are tabulated in Table 2.

From the table, we can see that the refractive index $n_{\mathrm{p}}$ of the cured film was larger than that of the corresponding monomer, with the increment of $0.03 \sim 0.05$ on the average. In our experiment, the $n_{\mathrm{o}}$ of LC E7 is 1.521. The number 3 monomer was the nearest to 1.521 and the experimental results proved that diffraction efficiency was the highest with the number 3 monomer. Similarly, for BL038, $n_{\mathrm{o}}$ is 1.527 . Only the number 2 monomer was the nearest to 1.528 and the experimental result proved that diffraction efficiency was the highest with the number 2 monomer. According to our selection principle, the number 3 and number 2 monomers match with E7 and BL038 liquid crystals, respectively.

\subsection{Effect of Nanoparticle Doping}

To extend H-PDLC applications, it is desirable to obtain higher index contrast. Doping nanoparticles with high refractive index into PDLC materials may be an easy and effective way to realize the higher index contrast. Some researchers have successfully demonstrated nanoparticle patterning, that is, holographic gratings, in nanoparticledispersed photopolymers using holography technique [9599]. In the nanoparticle-doped PDLC materials, the nanoparticles are anticipated to diffuse into the LC-rich regions during the phase separation between the polymer and LC. Therefore, the index contrast can be increased by this way.

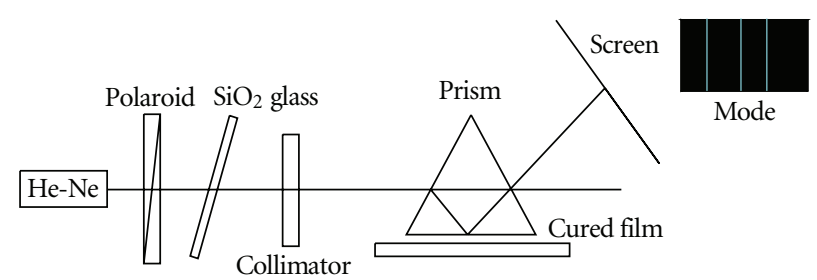

Figure 43: Measurement setup of the refractive index $n_{\mathrm{p}}$.

Jakubiak et al. studied the $\mathrm{ZnO}$ nanoparticles $(125 \mathrm{~nm}$ in diameter) doped H-PDLC gratings [100]. Figure 44 shows the SEM images of a transmission grating fabricated from $1 \% \mathrm{ZnO}$ and $36 \%$ LC with a grating period of about $500 \mathrm{~nm}$. The SEM images show rougher surface in the fractured cross section compared to the conventional $\mathrm{H}$ PDLC gratings without nanoparticle doping. As evidenced by the structural and performance characteristics of the nanoparticle/LC gratings, the particles are predominately located in the LC phase. The experimental results showed that the diffraction efficiency can be greatly enhanced for gratings containing $1 \%$ by weight of $\mathrm{ZnO}$ nanoparticles.

Kim et al. studied the nanosized silicas doped H-PDLCs [101]. The experimental results showed that the nanosized silicas doped H-PDLCs provide enhanced elasticity, dimensional stability, and diffraction efficiency. Figures 45(a) and 45(b) show the typical grating morphologies without and with silica filled, respectively. From the SEM images, the shrinkage volume is significantly decreased with the addition of filler. With decreased shrinkage, the LC-rich phase more or less keeps its width as grated. This implies that the rigidity of the resin phase holds the dimension of the grating close to the Bragg's spacings. This, together with an increased phase separation, should give high diffraction efficiency.

\section{Electro-Optical Properties}

\subsection{Field-Dependent Diffraction}

For H-PDLC-based devices, the most interesting thing is that they can be dynamically tuned or switched. Applying an electric field on H-PDLC is a very common way to control it. For H-PDLC gratings, in normal mode operation, the diffraction efficiency is high when the applied field is low and decreases with increasing field strength. Of course, there is an abnormal case when the polymer concentration is small (3-8 wt\%), so-called reversed mode [102, 103]. The "reversed mode" means that the incident light goes through the film without applied voltage and is diffracted with voltage applied. Figure 46 shows an example of reversed mode holographic polymer-stabilized liquid crystal $(\mathrm{H}-$ PSLC) grating.

Figure 47 shows the diffraction efficiency and transmission efficiency as a function of the applied electric field for a normal mode H-PDLC transmission grating. In Figure 47, the sum of the diffraction efficiency and transmission efficiency is also plotted. It can be seen that, the sum increases slightly with the electric field, which indicates a decrease 


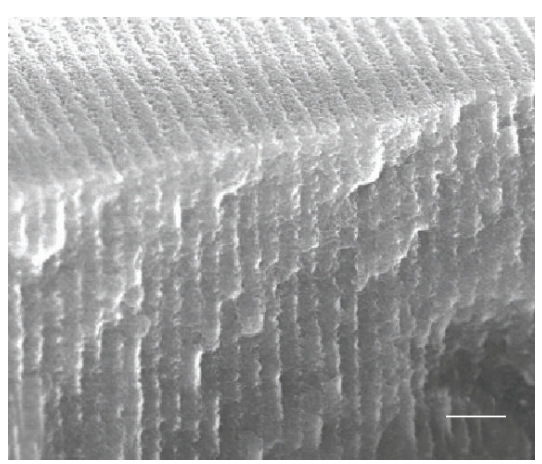

(a)

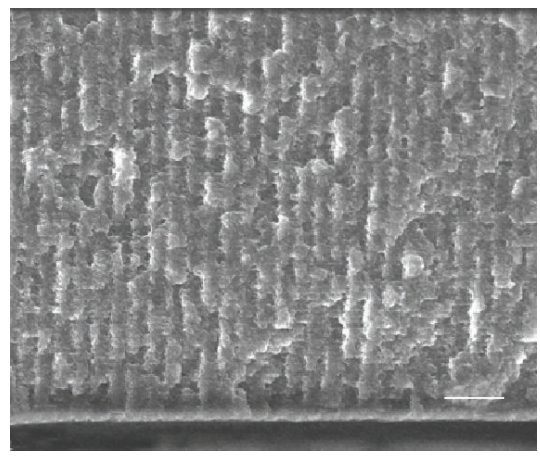

(b)

FIGURE 44: SEM micrographs of the (a) top and side and (b) side of a Bragg transmission grating. Note in (b) the cross grating appearing as horizontal lines. The scale bar corresponds to $1 \mu \mathrm{m}$ for both panels [100].

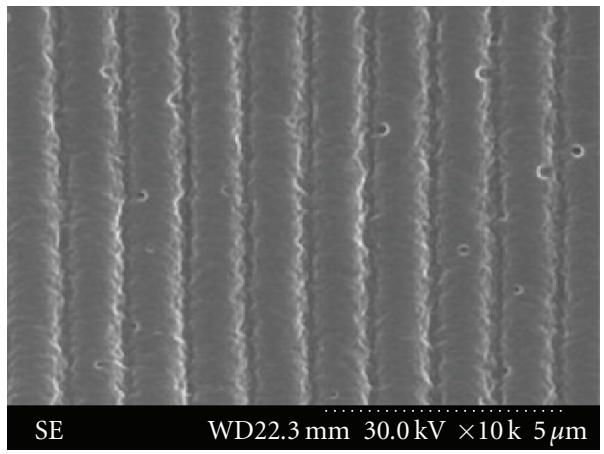

(a)

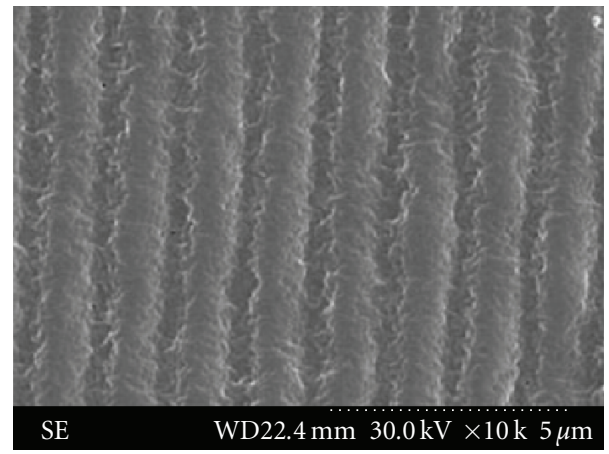

(b)

Figure 45: Grating morphology (oligomer/EHA = 3:1, 35 wt.- $\%$ LC): (a) without silica, $d=1.35 \mu \mathrm{m}$, and $h=0.58 \mu \mathrm{m}$, (b) with Aerosil 200, $d=1.47 \mu \mathrm{m}$ and $h=0.76 \mu \mathrm{m}[101]$.

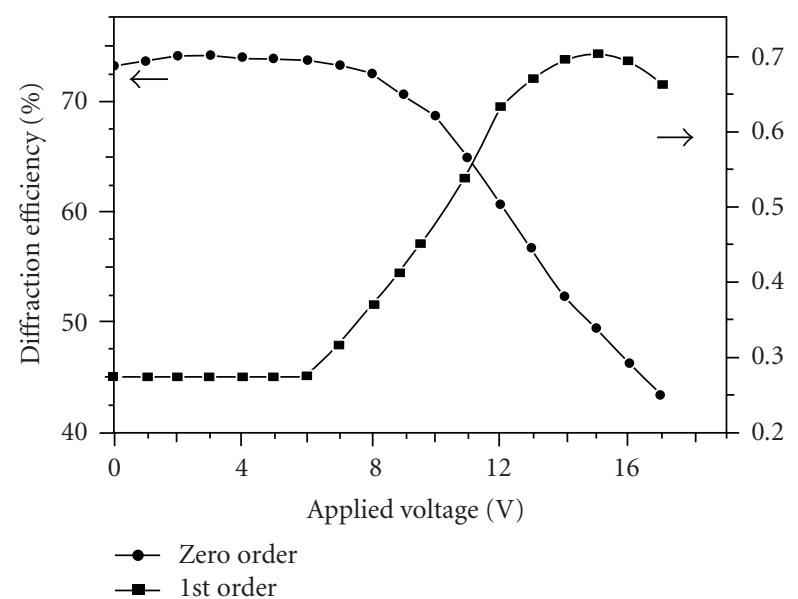

FIGURE 46: Diffraction efficiency as a function of applied voltage [103].

in the random scattering. When the diffraction efficiency is reduced to the minimum, the transmission efficiency reaches the maximum. Reported switching fields vary widely from $20 \mathrm{~V} / \mu \mathrm{m}$ [53] to $6 \mathrm{~V} / \mu \mathrm{m}$ [104], depending mostly on the materials system used to form the hologram. Lowering this value is of importance for practical applications and is a subject of continuing research.

In some cases, with the increase of applied voltage, the diffraction efficiency firstly increases and then decreases, as shown in Figure 48 for the curve with TPGDA monomer. Optical clearing, originating from droplet size distribution, may be the possible reason for the initial increase in diffraction efficiency. In H-PDLC gratings, large liquid crystal droplets tend to scatter more light, producing a haze effect. When an electric field is applied on the sample, these large droplets will align firstly due to the larger volume to surface area ratio, and the haze will disappear, thus, resulting in the diffraction efficiency increase initially. When the electric field continues to increase, the smaller droplets start to align, and the index modulation decreases. As a result, the diffraction efficiency will decrease. The similar behavior was also observed in H-PDLC PhCs in the following section. Above the switching electric field, with further increase in electric field, the diffraction efficiency increases again, as shown in Figure 49. From Figure 49, an increase of diffraction efficiency can be clearly observed when the electric field is above $27.3 \mathrm{~V} / \mu \mathrm{m}$. 


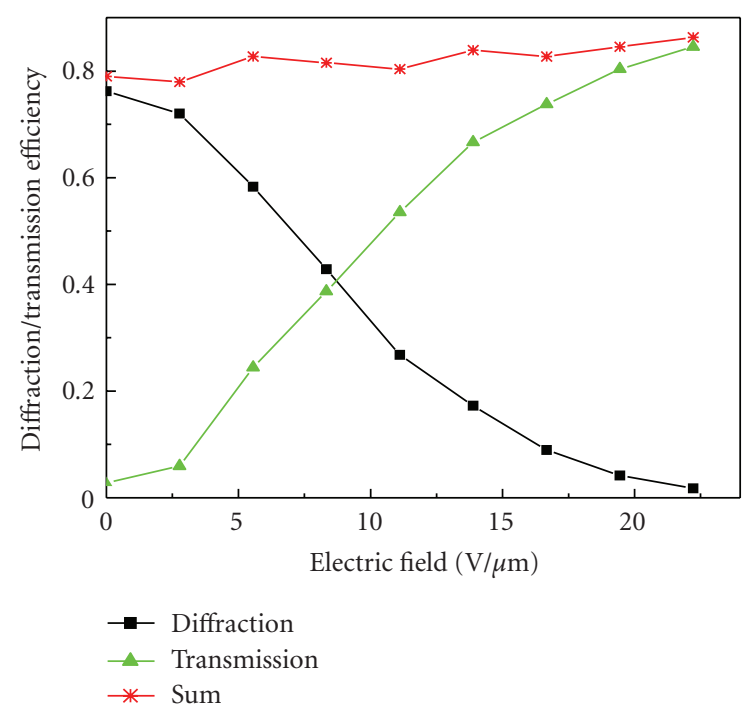

FIGURE 47: Diffraction and transmission efficiency as a function of applied electric field. The sum refers to the sum of diffraction and transmission efficiencies.

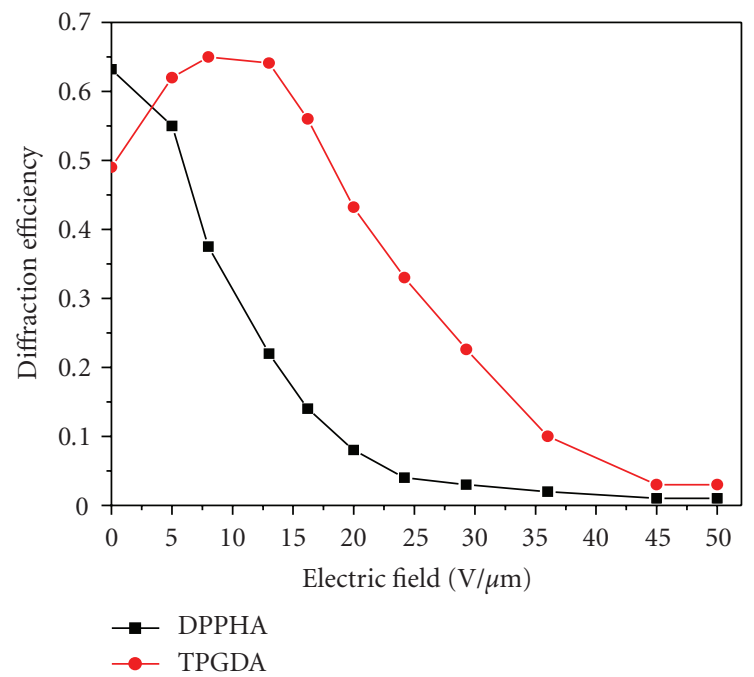

FIGURE 48: Diffraction efficiency as a function of applied electric field.

The general dynamic behavior for the diffraction efficiency under an external electric field can be explained in the following. Assuming the LC droplets in H-PDLCs are bipolar configuration, the LC droplets have an axis of symmetry along the long axis of the droplet, which can be then considered as a uniaxial domain with an angledependent extraordinary refractive index. Figure 50 shows the case when the LC droplets under an electric field, where $\theta_{\mathrm{B}}$ is the Bragg angle and $\theta(E)$ is the angle between the LC director direction and the electric field direction, which is a function of electric field and can be expressed as

$$
\theta(E)=\frac{1}{2} \tan ^{-1}\left[\frac{\sin 2 \theta_{0}}{\cos 2 \theta_{0}+\left(E / E_{c}\right)^{2}}\right],
$$

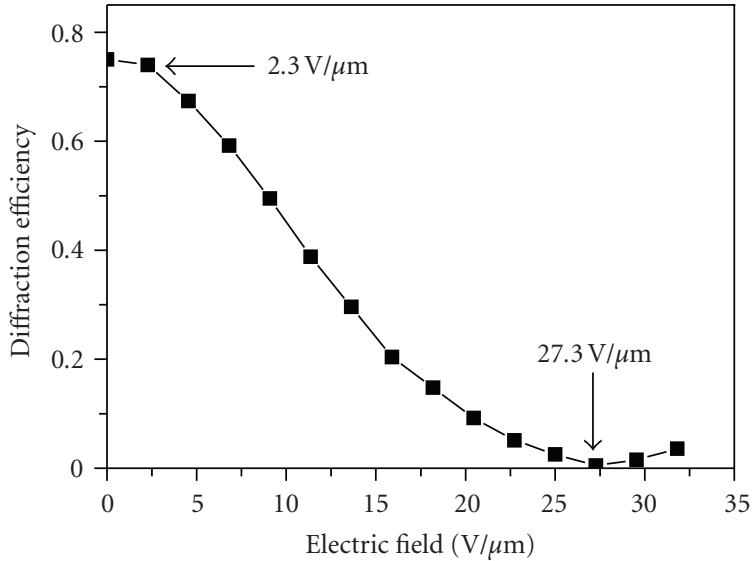

Figure 49: Diffraction efficiency as a function of the electric field. The threshold field and switching field are indicated.

where $\theta_{0}$ is the angle between the symmetry axis of LC droplets and the Bragg plane without voltage applied and $E_{c}$ is a critical field for switching which depends on properties of the LC droplet and surrounding polymer,

$$
E_{c}=\frac{1}{3 a}\left(\frac{\sigma_{\mathrm{LC}}}{\sigma_{p}}+2\right)\left[\frac{k_{33}\left(\ell^{2}-1\right)}{\Delta \varepsilon}\right]^{1 / 2},
$$

where $\sigma_{\mathrm{LC}}$ and $\sigma_{p}$ are the electrical conductivities of the LC and polymer, respectively, $k_{33}$ is the elastic bend force constant, and $\Delta \varepsilon$ is the dielectric anisotropy of the LC.

Initially, without voltage applied, the LC director statistically aligns along the grating vector director [83]. The p-polarized incident light with exact Bragg angle sees the largest refractive index difference between the polymerrich lamellae and the LC-rich lamellae and thus highest diffraction efficiency is obtained. When a voltage is applied on the sample, the LC director will start to change its direction along the electric field direction at a voltage, so-called threshold voltage. The incident light then sees the decreased index difference and hence the diffraction efficiency starts to decrease. With the increase of the voltage, when $\theta(E)=\theta_{\mathrm{B}}$, the incident light only sees the ordinary refractive index of the LCs, which is closest to the refractive index of polymer matrix. As a result, the incident light sees the smallest index difference between the polymerrich lamellae and the LC-rich lamellae and the diffraction efficiency decreases to the minimum value. With the further increase of the voltage, the LC director continues to align along the electric field direction with a smaller $\theta(E)$ and an index mismatch appears again. As a result, the incident light sees a larger index difference value again and the diffraction efficiency increases again.

It is worth mentioning that an H-PDLC grating itself is $2 \times 2$ optical switch [38]. With an optimized ratio of various materials, the highest diffraction efficiency achieved at $632.8 \mathrm{~nm}$ was $85.7 \%$. The corresponding contrast ratio 

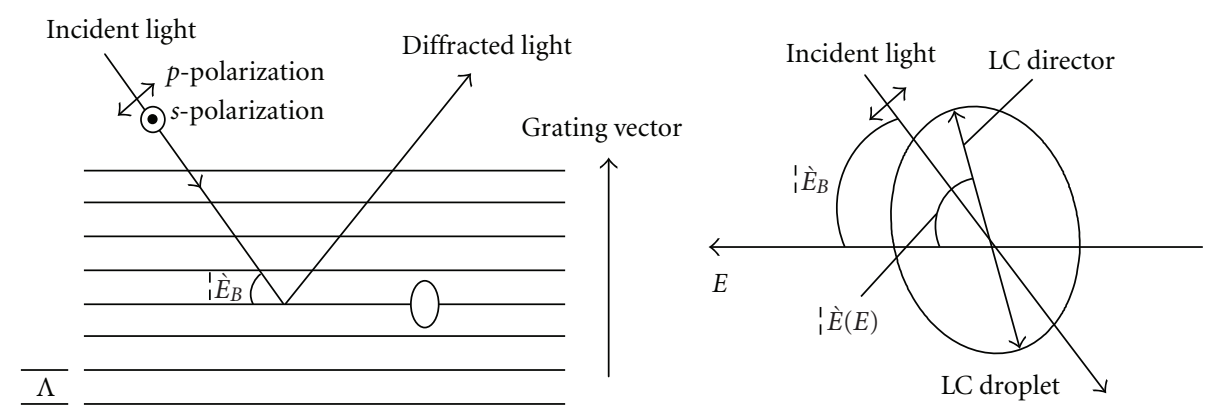

FIgURE 50: LC droplets under an external electric field.

(CR) and signal-noise ratio (SNR) were about $23.4 \mathrm{~dB}$ and $15.1 \mathrm{~dB}$, respectively, where CR and SNR can be defined as

$$
\begin{aligned}
\mathrm{CR} & =10 \log \left(\frac{D E_{\max }}{D E_{\min }}\right) \quad \text { or } \quad \mathrm{CR}=10 \log \left(\frac{T E_{\max }}{T E_{\min }}\right), \\
\mathrm{SNR} & =10 \log \left(\frac{D E_{\max }}{T E_{\min }}\right) \quad \text { or } \quad \mathrm{SNR}=10 \log \left(\frac{T E_{\max }}{D E_{\min }}\right),
\end{aligned}
$$

where $D E_{\max }$ is the highest diffraction efficiency, $D E_{\min }$ is the lowest diffraction efficiency, $T E_{\max }$ is the highest transmission efficiency, and $T E_{\min }$ is the lowest transmission efficiency.

The index difference plays a vital role in determining the performance of the optical switch. We can optimize the performance of the optical switch according to the following considerations.

\section{(1) Decreasing the Residual Diffraction}

This demands us to choose suitable liquid crystal and polymer, which makes the ordinary refractive index of liquid crystal and the refractive index of polymer as close as possible. Theoretically, only when the ordinary refractive index of liquid crystal strictly equals to the refractive index of polymer, there is no residual diffraction. In fact, it is impossible to eliminate the residual diffraction. However, it is still possible to decrease the residual diffraction. As known, it is difficult to modify the refractive index of polymer. However, it is quite flexible to modify the refractive index of liquid crystals. An easy way is to mix different liquid crystals to achieve the ordinary refractive index with a value closer to the refractive index of polymer.

\section{(2) Controlling the Phase Separation}

It is impossible to obtain an ideally perfect phase separation in H-PDLCs. In fact, during the process of phase separation, many LC molecules (polymers) are trapped in the polymerrich lamellae (LC-rich lamellae) when the counter diffusion happens, which decreases the index difference between polymer-rich lamellae and LC-rich lamellae and thus the diffraction efficiency.

\section{(3) Controlling the Size of LC Droplets}

The size of LC droplets also plays an important role to improve the diffraction efficiency. Some LC droplets in polymer-rich lamellae act as scatterers and thus decrease the diffraction efficiency. Smaller size of LC droplets can reduce the residual scattering and therefore increase the diffraction efficiency.

\subsection{Response Time}

The on time, $\tau_{\text {on }}$, is the time required for an H-PDLC to switch diffraction between orders in response to a step electric field. This corresponds to a decrease in diffraction in a normal mode operation, an increase in diffraction in a reverse mode operation, or a lowering of the frequency when using frequency addressed H-PDLC. Conversely, the off time, $\tau_{\text {off, }}$, is the time required for an H-PDLC to change diffraction in response to a sudden removal of an electric field. A typical time response for an H-PDLC Bragg grating is shown in Figure 51 . The $\tau_{\text {on }}$, measured from the rising edge of the voltage pulse, is about $36 \mu$ s. The $\tau_{\text {off }}$, measured from the fall edge of the voltage pulse, is about $160 \mu$ s.

Another interesting thing to note is that, in Figure 51(b), an optical bounce is clearly observed immediately after the electric field is applied. It is probably due to the backflow effect of liquid crystals $[105,106]$. Backflow effect is a consequence of flow close to the LC cell walls induced by the rapidly relaxing alignment following the removal of the electric field, which generally exists in the TN cells. This flow essentially creates a shear flow in the center of the cell, which causes a temporary reversal of the relaxation in that region, before the alignment finally relaxes as one anticipates. As a result, an optical bounce in transmission curve is experimentally observed when the light is normally incident on the cell, which is induced by the backflow effect. In our experiments, this phenomenon was only observed at the edge of "on" state. We speculate the reason as follows. In HPDLCs, the nematic configurations in most LC droplets are generally bipolar, as shown in Figure 10(a). When an external electric field is applied on H-PDLCs, the nematic director realigns along the electric field direction. At the same time, an internal electric field is induced due to the impurities (mobile ions) in H-PDLCs, which thus decreases the external electric field. Then due to the balance between the elastic torque and 


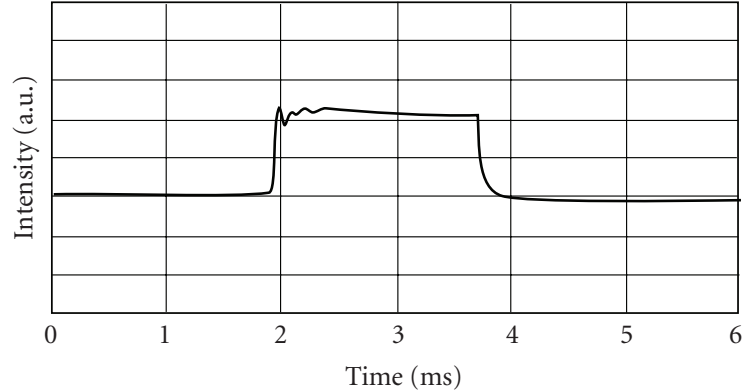

(a)

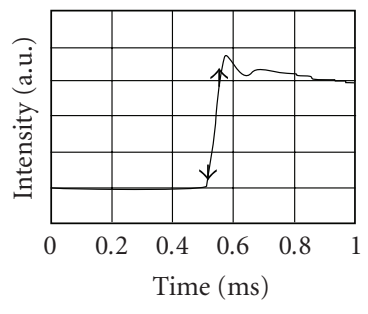

(b)

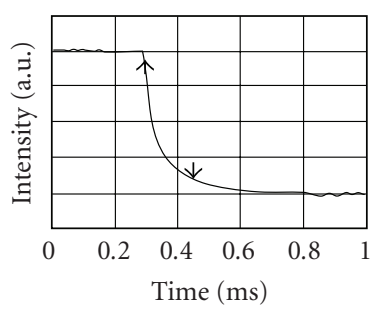

(c)
FIGURE 51: Variation of diffracted light intensity corresponding to (a) a square driving voltage, (b) the magnified rising edge, and (c) the falling edge of (a).

the electric torque in the LC droplets, and the delay between the external electric field and the induced ion field, an optical bounce in transmission curve was observed at the edge of "on" state. After the removal of the external electric field, the nematic director configurations gradually revert to the original status due to the elastic torque, where the optical bounce would be difficult to be observed at the edge of "off" state.

For acrylate-based H-PDLCs, reported values of $\tau_{\text {on }}$ vary from $25 \mu \mathrm{s}$ [107] to $750 \mu$ s [35] and the reported $\tau_{\text {off values }}$ are slightly longer, ranging from $44[107,108]$ to $1000 \mu \mathrm{s}$ [109]. For the thoil-ene-based H-PDLCs, the reported values of $\tau_{\text {on }}$ vary from $550 \mu$ s to $6.7 \mathrm{~ms}$ and the reported $\tau_{\text {off }}$ values vary from 1.2 milliseconds to 2.2 milliseconds [48, 110, 111], which are generally much longer than those of the acrylatebased H-PDLCs. This is expected from Wu's model [86] since there is no field to drive the LC back to the zero field state. They expressed $\tau_{\text {off }}$ and $\tau_{\text {on }}$ as

$$
\begin{aligned}
\tau_{\text {off }} & =\frac{\gamma_{1} a^{2}}{k_{33}\left(\ell^{2}-1\right)}, \\
\tau_{\text {on }} & =\frac{\gamma_{1}}{\Delta \varepsilon E^{2}+k_{33}\left(\ell^{2}-1\right) / a^{2}},
\end{aligned}
$$

where $\gamma_{1}$ is the rotational viscosity coefficient of LC.

As can be seen from (48), the off time is mainly controlled by elastic forces for most PDLC-based devices. Most experimental results show that the off time is generally much slower than the on time. To improve the off time, Kitzerow developed dual frequency H-PDLCs, which were electrically driven at both an on and off state [113]. Using a dual-frequency LC where an electric field is employed to turn the grating off, the switching times can be much improved to a few milliseconds. Compared to conventional nematicpositive dielectric LCs where the $100 \mathrm{~ms}$ was typically obtained when turning off the voltage, this off speed is faster. Due to the large period the gratings where large LC droplets existed, these speeds are slow compared to those of conventional Bragg gratings. However, it showed a good method to improve the response time.

\section{Applications}

In the last several years, new applications have been developed based on H-PDLC, mainly in three aspects: (i) H-PDLC photonic crystals (PhCs), (ii) mirrorless lasing from dyedoped H-PDLC, and (iii) sensing applications. It is believed that H-PDLC will have additional applications in photonics or other fields.

\subsection{Photonic Crystals}

Recently, much interest was focused on fabricating $\mathrm{PhCs}$ based on H-PDLC. PhCs refers to those structures with periodic dielectric materials in the optical range, where light in special frequency range is forbidden in the photonic bandgap. As a new class of photonic bandgap material, combining the holography technique and electrically controlled switchability, H-PDLC PhCs showed many advantages over conventional $\mathrm{PhCs}$, including single-step and easy fabrication, field-dependent switchability, and fast response. Tondiglia et al., firstly fabricated H-PDLC PhCs in 2002 [39]. $\mathrm{H}$-PDLC gratings (transmission or reflection mode) can be viewed as the simplest $1 \mathrm{D}$ PhCs. Many $2 \mathrm{D}$ and $3 \mathrm{D} \mathrm{PhCs}$, have also been demonstrated using H-PDLC materials, including orthorhombic (Figure 52) [112], face-centered cubic (Figure 53) [114, 116], transverse square (Figure 54) [115, 117], diamond-like lattices [118], and Penrose structure [119]. Theoretically and experimentally, using three and four coherent beams, five 2D and all fourteen 3D Bravais lattices can be formed using H-PDLC materials, respectively [120]. With five or more beams, even more complex quasicrystal structures were also investigated $[41,121]$. Enhanced diffraction efficiency can be achieved by nanoparticle doped $\mathrm{H}$ PDLC PhCs [122]. Generally, to achieve these structures, a relatively complicated optical setup is generally used to create multibeam interference pattern in all these fabrications. However, it is possible to reduce the optics complexity and fabricate the complicated structures. Only using a singlediffraction element, a photomask, which creates a threebeam interference pattern, we successfully demonstrated 2D H-PDLC PhCs [40]. Using a single prism with top-cutting, 2D and 3D H-PDLC PhCs were also fabricated [123, 124]. This single element implementation improves the alignment and stability of the optical setup, making it more robust than the multiple beam setups reported previously.

Figures 55(a) and 55(b) show the schematic of the mask and three diffracted beams to fabricate 2D H-PDLC PhCs only using a photomask. When a collimated $\mathrm{Ar}^{+}$ laser beam impinges on the mask, which produces three diffracted beams, a diffraction pattern will be produced at 


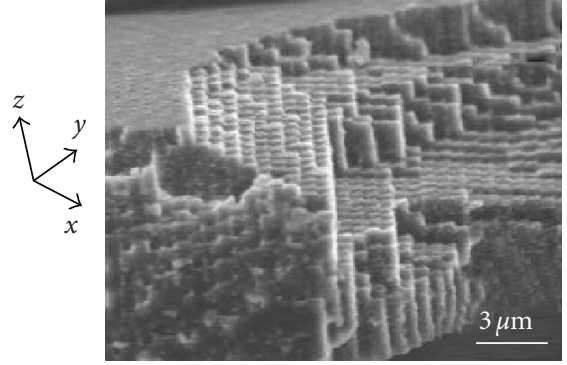

(a)

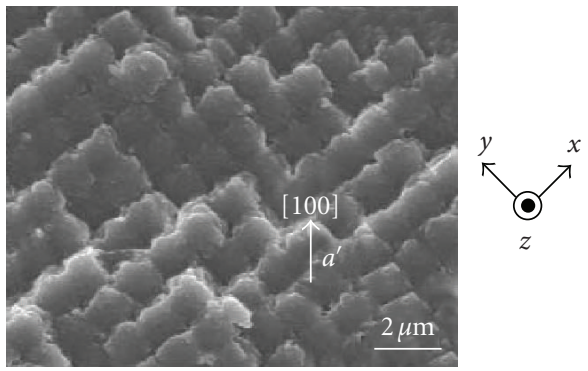

(b)

Figure 52: SEM images of sample fabricated in the sp configuration with $\theta_{i}=45^{\circ}, \theta=17.28^{\circ}$. (a) 3D perspective. (b) Top view [112].

their intersection region. In our experiment, each grating on the mask has an area of $8 \times 8 \mathrm{~mm}^{2}$ and the grating period is $4 \mu \mathrm{m}$. A cell filled with the $\mathrm{LC} /$ prepolymer mixture is exposed to the diffraction pattern. The LC and polymer will redistribute because of the polymerization of the prepolymer induced by the light intensity, thus forming a $2 \mathrm{D}$ structure inside the cell.

The electrical field distribution of multibeam interference can be generally described by

$$
\begin{aligned}
I(\mathbf{r})= & {\left[\sum_{j=1}^{n} \mathbf{E}_{j}(\mathbf{r}) \exp \left(i \mathbf{k} \cdot \mathbf{r}+i \phi_{j}\right)\right] } \\
& \times\left[\sum_{j=1}^{n} \mathbf{E}_{j}^{*}(\mathbf{r}) \exp \left(-i \mathbf{k} \cdot \mathbf{r}+i \phi_{j}\right)\right] \\
= & \sum_{j=1}^{n}\left|\mathbf{E}_{j}\right|^{2}+\sum_{i \neq j}^{n} \mathbf{E}_{i} \cdot \mathbf{E}_{j}^{*} \exp \left[i\left(\mathbf{k}_{i}-\mathbf{k}_{j}\right) \cdot \mathbf{r}+i \phi_{i j}\right],
\end{aligned}
$$

where $\mathbf{E}$ is the amplitude, $\mathbf{k}$ is the wave vector, $i$ and $j$ are integers, $\phi_{i j}$ is the initial phase difference between two incident waves, and $\mathbf{r}$ is the position vector. The orientation of the wave vector of the three beams can be defined as

$$
\begin{aligned}
& \hat{\mathbf{k}}_{1}=\sin \theta \cos \varphi_{1} \hat{\mathbf{e}}_{x}+\sin \theta \sin \varphi_{1} \hat{\mathbf{e}}_{y}+\cos \theta \hat{\mathbf{e}}_{z}, \\
& \hat{\mathbf{k}}_{2}=\sin \theta \cos \varphi_{2} \hat{\mathbf{e}}_{x}+\sin \theta \sin \varphi_{2} \hat{\mathbf{e}}_{y}+\cos \theta \hat{\mathbf{e}}_{z}, \\
& \hat{\mathbf{k}}_{3}=\sin \theta \cos \varphi_{3} \widehat{\mathbf{e}}_{x}+\sin \theta \sin \varphi_{3} \widehat{\mathbf{e}}_{y}+\cos \theta \widehat{\mathbf{e}}_{z},
\end{aligned}
$$

where $\hat{\mathbf{k}}$ is unit wave vector, $\hat{\mathbf{e}}$ is unit coordinate vector, $\theta$ is the angle between the laser beam and $z$-axis, which equals the first-order diffraction angle, and $\varphi$ is the angle between the projection of the laser beam on the $x-y$ plane and the $x$ axis (see Figure 55). Substituting (51)-(53) into (50) with $\theta$ $=7.4^{\circ}, \varphi_{1}=180^{\circ}, \varphi_{2}=-60^{\circ}$, and $\varphi_{3}=60 \circ$, we have

$$
\begin{aligned}
I= & \left(\mathbf{E}_{1}+\mathbf{E}_{2}+\mathbf{E}_{3}\right) \cdot\left(\mathbf{E}_{1}+\mathbf{E}_{2}+\mathbf{E}_{3}\right)^{*} \\
= & \left|\mathbf{E}_{1}\right|^{2}+\left|\mathbf{E}_{2}\right|^{2}+\left|\mathbf{E}_{3}\right|^{2} \\
& +2 \mathbf{E}_{1} \cdot \mathbf{E}_{2} \cos \left(-\frac{3}{2} k x \sin \theta+\frac{\sqrt{3}}{2} k y \sin \theta\right) \\
& +2 \mathbf{E}_{1} \cdot \mathbf{E}_{3} \cos \left(-\frac{3}{2} k x \sin \theta-\frac{\sqrt{3}}{2} k y \sin \theta\right) \\
& +2 \mathbf{E}_{2} \cdot \mathbf{E}_{3} \cos (\sqrt{3} k y \sin \theta) .
\end{aligned}
$$

The simulated 3D interference pattern is shown in Figure 56. It is worth mentioning that the period of this triangular lattice is equal to two thirds of the diffraction grating period, that is, $2.67 \mu \mathrm{m}$, which does not depend on the beam wavelength [125]. Therefore, the PBGs can be easily engineered by changing the grating period.

Figure 57(a) shows an AFM image of the surface, which was obtained after the liquid crystals were removed in ethanol. It reveals a clear hexagonal morphology with a lattice constant of about $2.5 \mu \mathrm{m}$, which is in good agreement with the simulation pattern (Figure 56), considering the general $5-10 \%$ volume shrinkage for the acrylate monomer during the photo-induced polymerization. It is interesting to note in Figure 57(a), in a hexagonal cell, the height of the central point is slightly different from its surrounding six points, which are almost the same in height. Figure 57(b) shows the surface morphologies of the 2D H-PDLC PhCs observed under a high-resolution optical microscope (HiROX). The inset SEM image in Figure 57(b) shows the cross-section morphology of the sample, where columnar structure is clearly seen, which matches the simulation result in Figure 56.

The diffraction patterns of the H-PDLC PhCs were checked to confirm the quasicrystal structure. Figures 58(a) and 58 (b) show the visible diffraction pattern produced by a normally incident He-Ne laser beam operating at $543 \mathrm{~nm}$ and a collimated broadband white beam for our sample. From Figure 58(a), we can see clear hexagonal diffraction spots which match well with our AFM, optical, and SEM images of a 2D hexagonal lattice (Figure 57). The diffracted spots originating from $\left(\begin{array}{lll}0 & 1 & \overline{1}\end{array}\right)$ and $\left(\begin{array}{lll}1 & 1 & \overline{2}\end{array}\right)$ surfaces are labeled in Figure 58(a). The images clearly reveal the presence of quasiperiodicity within the sample. The observed points are sharp and symmetrically distributed. As reported by Gorkhali et al. [41], all diffraction points in the first and higher orders should show an $N$-fold symmetry and have $2 N$ diffracted points, where $N$ is the number of interference beams used to produce the PhC. For our case, $N=3$, in good agreement with the prediction, we can clearly see a 3fold symmetry and 6 diffracted points for the same order of diffracted beams.

3D H-PDLC PhCs can be fabricated using a top-cup prism, as shown in Figure 59(a). A laser beam is collimated first and then impinges normally onto the specially designed 


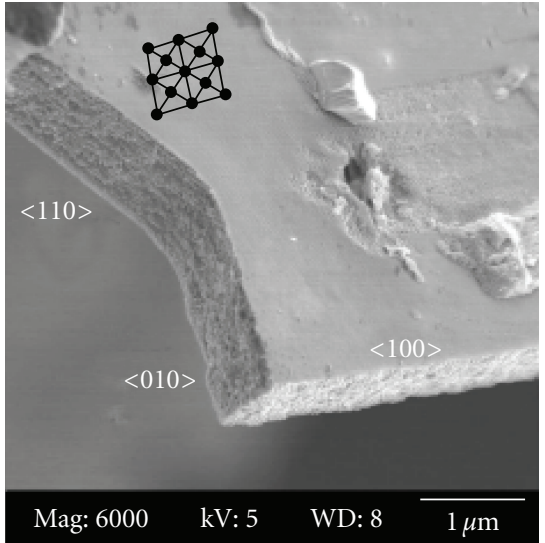

(a)

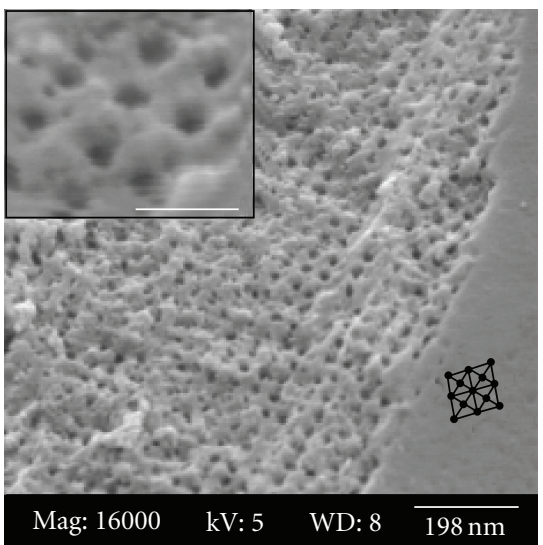

(c)

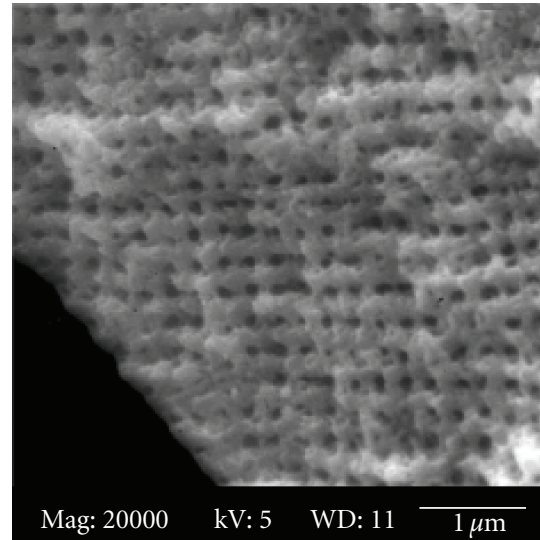

(b)

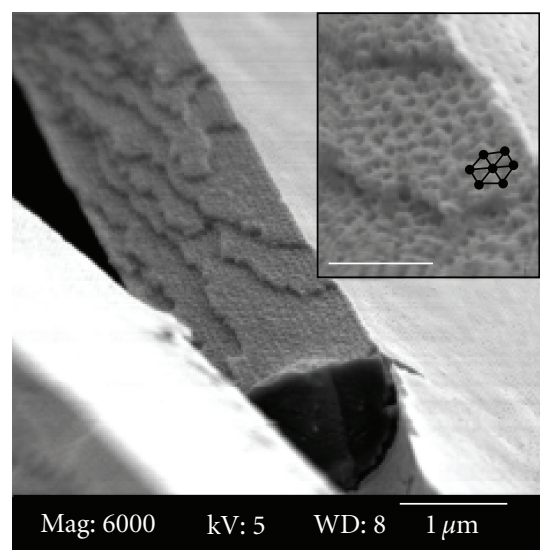

(d)

Figure 53: SEM images of the fcc lattice in H-PDLCs: (a) macroscopic fracture planes follow the mesoscopic crystal lattice directions; (b)

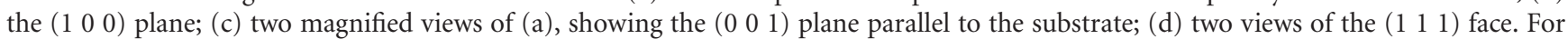
both (c) and (d) the scale bars of the insets correspond to $200 \mathrm{~nm}$ [114].

prism, which is made of BK7 glass. The side lengths of the top- and bottom-surfaces (isosceles triangle) are 1 and $6 \mathrm{~cm}$, respectively, and the height is $2.5 \mathrm{~cm}$. Emerging from the prism are one directly transmitted beam $\mathbf{k}_{0}$ in the center and three side beams, $\mathbf{k}_{1}, \mathbf{k}_{2}$, and $\mathbf{k}_{3}$, by the refraction from three tilted side surfaces of the prism, as shown in Figure 59(b). These four beams overlap at the bottom surface of the prism and interfere with each other, forming a 3D intensity pattern. The light intensity pattern can be described by

$$
\begin{aligned}
I= & \left(\mathbf{E}_{0}+\mathbf{E}_{1}+\mathbf{E}_{2}+\mathbf{E}_{3}\right) \cdot\left(\mathbf{E}_{0}+\mathbf{E}_{1}+\mathbf{E}_{2}+\mathbf{E}_{3}\right)^{*} \\
= & \left|\mathbf{E}_{0}\right|^{2}+\left|\mathbf{E}_{1}\right|^{2}+\left|\mathbf{E}_{2}\right|^{2}+\left|\mathbf{E}_{3}\right|^{2} \\
& +2 \mathbf{E}_{0} \cdot \mathbf{E}_{1} \cos (k x \sin \theta+k z-k z \cos \theta) \\
& +2 \mathbf{E}_{0} \cdot \mathbf{E}_{2} \cos \left(-\frac{1}{2} k x \sin \theta+\frac{\sqrt{3}}{2} k y \sin \theta+k z-k z \cos \theta\right) \\
& +2 \mathbf{E}_{0} \cdot \mathbf{E}_{3} \cos \left(-\frac{1}{2} k x \sin \theta-\frac{\sqrt{3}}{2} k y \sin \theta+k z-k z \cos \theta\right) \\
& +2 \mathbf{E}_{1} \cdot \mathbf{E}_{2} \cos \left(-\frac{3}{2} k x \sin \theta+\frac{\sqrt{3}}{2} k y \sin \theta\right)
\end{aligned}
$$

$$
\begin{aligned}
& +2 \mathbf{E}_{1} \cdot \mathbf{E}_{3} \cos \left(-\frac{3}{2} k x \sin \theta-\frac{\sqrt{3}}{2} k y \sin \theta\right) \\
& +2 \mathbf{E}_{2} \cdot \mathbf{E}_{3} \cos (\sqrt{3} k y \sin \theta) .
\end{aligned}
$$

From (55), the periods $a, b$, and $c$ along the $x, y$, and $z$ directions depend on the angle $\theta$

$$
\begin{aligned}
& a=\frac{\lambda_{w}}{\sqrt{3} n_{\mathrm{eff}} \sin \theta}, \\
& b=\frac{\lambda_{w}}{n_{\mathrm{eff}} \sin \theta}, \\
& c=\frac{\lambda_{w}}{2 n_{\mathrm{eff}}(1-\cos \theta)},
\end{aligned}
$$

where $\lambda_{w}$ is the writing wavelength, and $n_{\text {eff }}$ is the effective refractive index of the recording materials. In our experiment, $\lambda_{w}=514.5 \mathrm{~nm}$, assuming $n_{\text {eff }}=1.59$ according to the LC concentration in the homogeneous PDLCs, $\theta=24.1^{\circ}$, we can theoretically obtain $a=458 \mathrm{~nm}, b=792 \mathrm{~nm}$, and $c=$ $1856 \mathrm{~nm}$. 


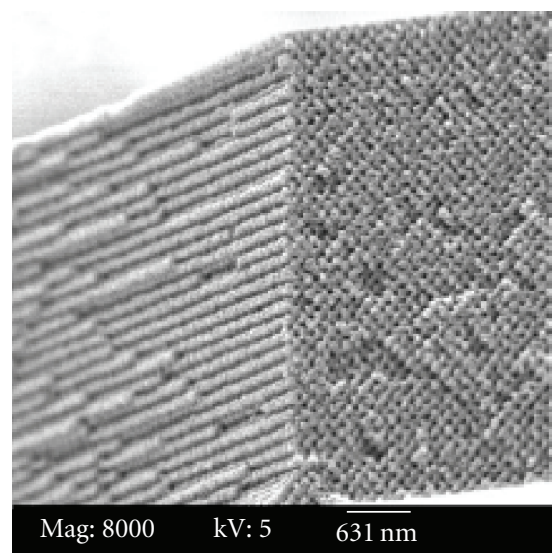

(a)

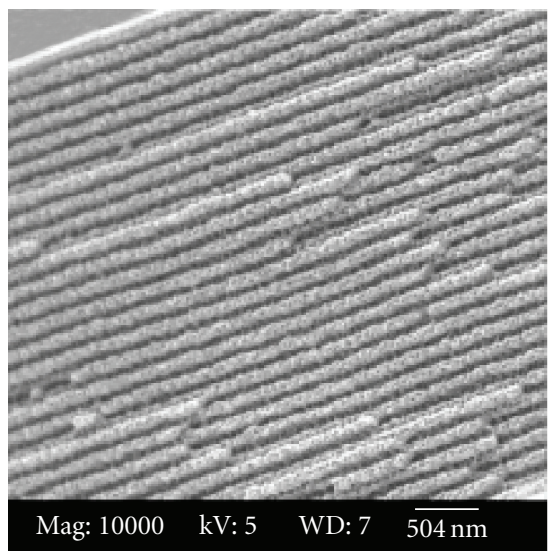

(c)

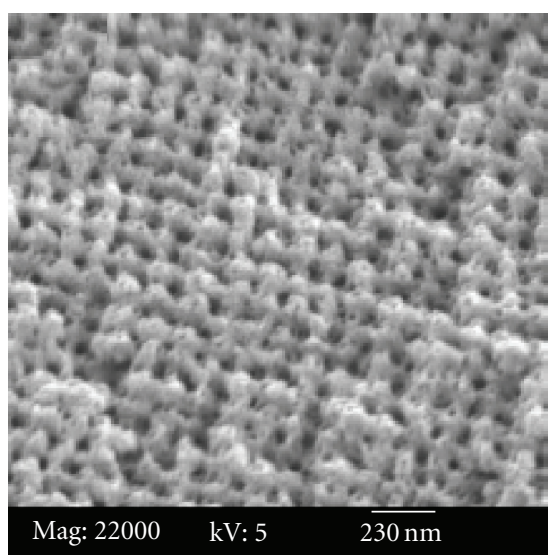

(b)

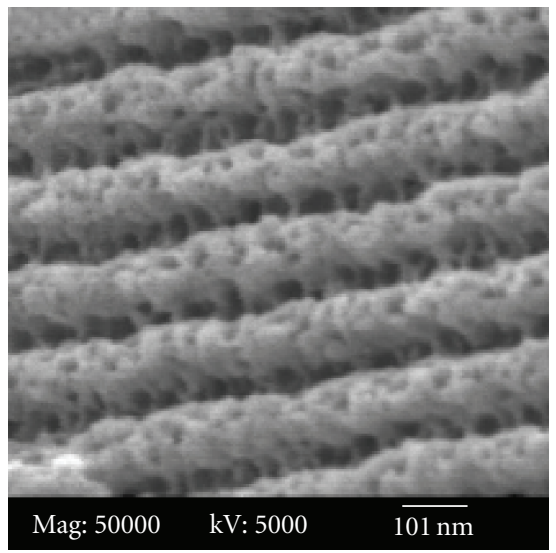

(d)

FIGURE 54: SEM images. (a) Low magnification; (b) closeup of $X Z$-face viewed from a slightly different perspective than in (a); (c) side view of cylindrical LC cavities; (d) closeup of LC cavities [115].

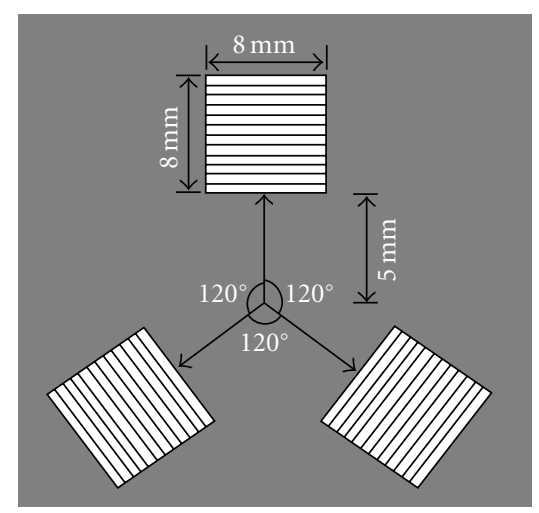

(a)

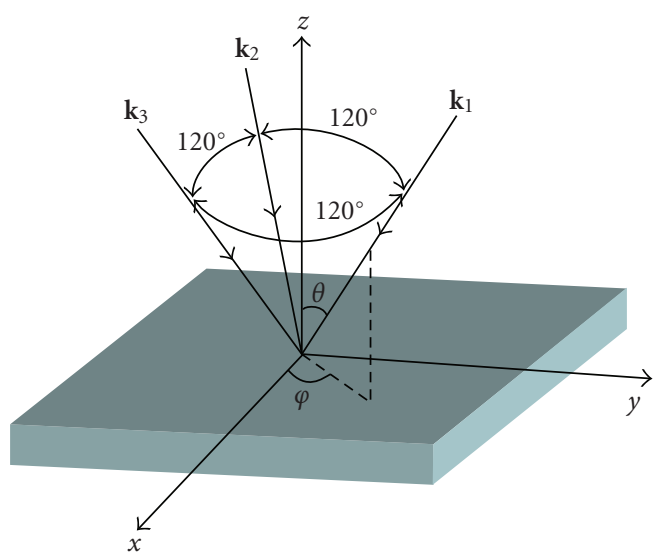

(b)

FIGURE 55: (a) Schematic of the mask and (b) three first-order diffracted beams. 


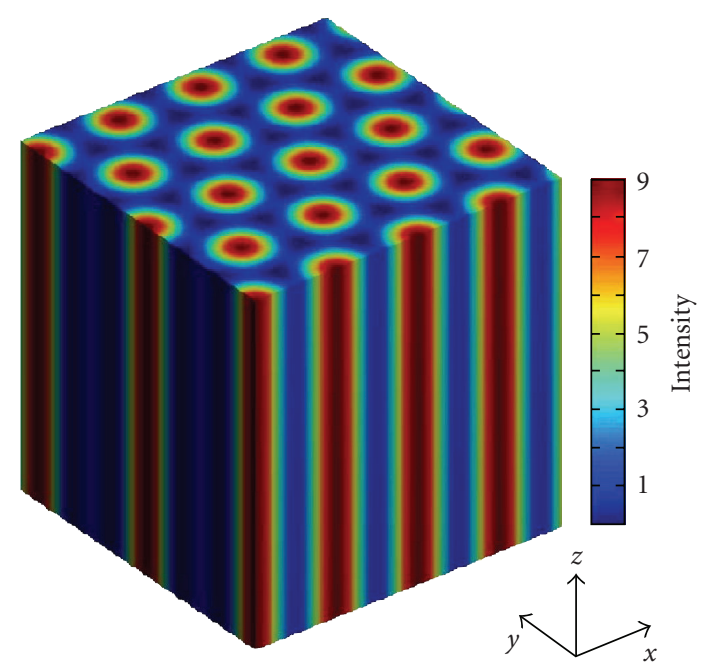

Figure 56: Simulated 3D interference pattern. The color bar shows the intensity distribution.

With the 4-beam intensity ratio of 3:1:1:1 estimated, Figure 60 shows the theoretically calculated 3D pattern of the spatial intensity distribution of the four-beam interference. Therefore, the PBGs can be easily engineered by changing the cutting angle of the prism.

In our experiment, the intensities of the four beams $\mathbf{k}_{0}$, $\mathbf{k}_{1}, \mathbf{k}_{2}$, and $\mathbf{k}_{3}$ are $58,21,22$, and $21 \mathrm{~mW} / \mathrm{cm}^{2}$, respectively, which is very close to the estimated ratio. Figures $61(\mathrm{a})$ and 61(b) show the AFM images of the surface morphologies with different scanning areas of $10 \times 10 \mu \mathrm{m}^{2}$ and $4 \times 4 \mu \mathrm{m}^{2}$, respectively. Figures 62 (a) and 62(b) show the SEM images of the surface and the cross-section for the sample, respectively, which are in good agreement with the simulation pattern. It can be seen from Figure 62 that the surface structure is a triangular lattice with a lattice constant of about $420 \pm 20 \mathrm{~nm}$, which is in good agreement with the theoretical calculation according to the geometrical structure (Figure 60).

Figures 63(a) and 63(b) show the visible diffraction pattern produced by a normally incident He-Ne laser beam operating at $543 \mathrm{~nm}$ and a broadband white beam for our $\mathrm{H}$-PDLC PhC sample fabricated. From Figure 63(a), we can see that three diffracted points are reconstructed when a laser beam is normally incident on the sample. It is worth mentioning that the three ellipse light spots in Figure 63(a) are induced by the edges of the top surface of the prism. A Kossel ring is also observed in the center of the diffraction pattern, which may be caused by the interference between the impinging four beams and the scattered light. Due to a decreased lattice constant, the white light diffraction exhibits a much larger dispersion (Figure 63(b)) compared to that of the 2D H-PDLC PhCs (Figure 58(b)).

Figure 64 shows the changes of the diffraction and the transmission intensity as functions of applied voltage. From Figure 64, we can see that, with the increase of applied voltage, the diffraction intensity decreases, while the transmission intensity increases, due to the refractive indices matching between the liquid crystal and polymer matrix. The switching electric field is about $7.2 \mathrm{~V} / \mu \mathrm{m}$. Due to the large LC concentration used in our experiment, the driving voltage decreases a great deal compared to the low LC concentration case.

Figure 65 shows the measured electro-optical response time when the sample was driven by a square voltage of $185 \mathrm{~V}_{\mathrm{rms}}(6.2 \mathrm{~V} / \mu \mathrm{m})$ with a frequency of $20 \mathrm{~Hz}$. From Figure 65 , the rise time (10\%-90\% intensity changed) and the fall time (90\%-10\% intensity changed), are about 1.75 and 1.09 milliseconds, respectively. The response time is relatively slow due to the large LC concentration. With a decrease in the LC concentration, the response time can be expected to be smaller.

For H-PDLC PhCs, the small refractive index contrast between liquid crystal and polymer matrix hinders their applications. The general refractive index difference can be about $0-0.4$, mainly depending on the LC materials. However, it is still possible to find applications where large index contrast is not needed, such as superprism effect. Li et al. has observed the superprism phenomenon based on 2D H-PDLC structures [126]. When a TE-polarized white light is incident onto an H-PDLC film, it is dispersed to yield a continuous spectrum distributed from red to blue, with an angle $\sim 50^{\circ}$. Jakubiak et al. demonstrated dynamic lasing from dye-doped 2D H-PDLC PhCs [42]. On the other hand, due to the anomalous dispersion characteristics at the band edge of PhCs, larger optical nonliearities can be produced in PhCs. A dramatic enhancement in the efficiency of thirdharmonic generation (THG) has been observed in $1 \mathrm{D} \mathrm{H}$ PDLC PhCs when the fundamental wavelength approaches the low-energy edge of the first photonic band gap [127].

\subsection{Mirrorless Lasing}

The dye-doped H-PDLC grating (either reflection or transmission mode) itself serves as both a resonant cavity and a gain medium, making it promising in electrically tunable lasers with low threshold. Since 2003, Jakubiak et al. demonstrated distributed feedback (DFB) lasing from dye-doped H-PDLC reflection grating [128]. Lucchetta et al. reported lasing emission from a simple wafer structure using an H-PDLC reflection grating as a reflection mirror [129] and later achieved light amplification in dye-doped H-PDLC reflection grating [130]. Much work has been done in the past two years about dye-doped H-PDLC lasing effect [49, 131-133]. The lasing emission can also be obtained using H-PDLC grating only as a distributed feedback element by $\mathrm{He}$ and his coworkers [134]. Most of above work regarding the lasing emission are based on $\mathrm{H}-\mathrm{PDLC}$ reflection gratings. However, in such cases, the overall gain length is limited to the thickness of the film. To increase the lasing efficiency, it is desirable to increase the film thickness. However, in reflection geometry, an increase of film thickness results in an increase of the required switching electric field. Thus, a tradeoff between cavity length and switching field is necessary. This problem can be overcome if the H-PDLC transmission mode grating is 


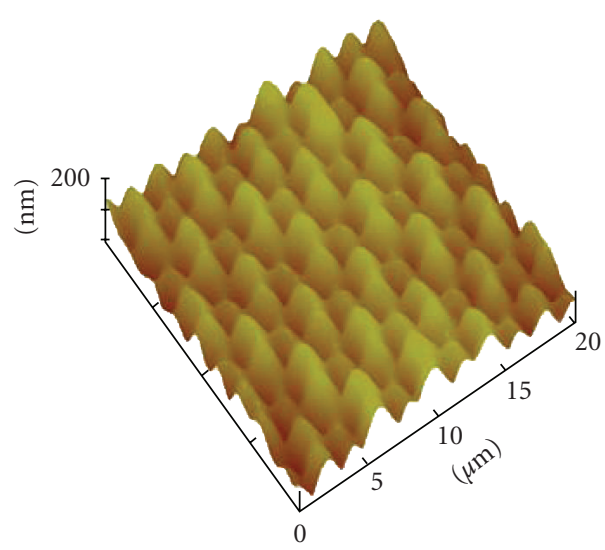

(a)

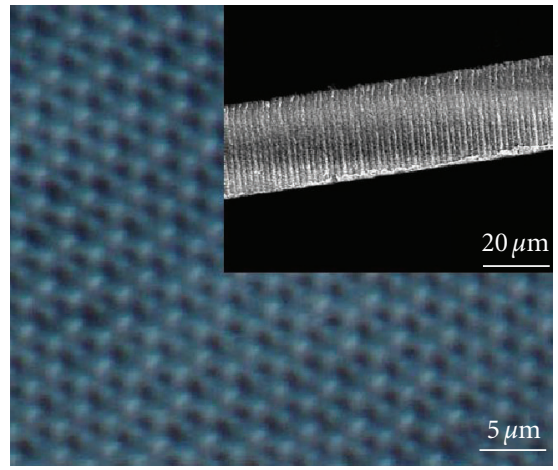

(b)

FIGURE 57: (a) AFM and (b) optical microscopy images showing the surface morphology of the 2D H-PDLC PhC and SEM image (the inset of (b)) showing the cross-section morphology.

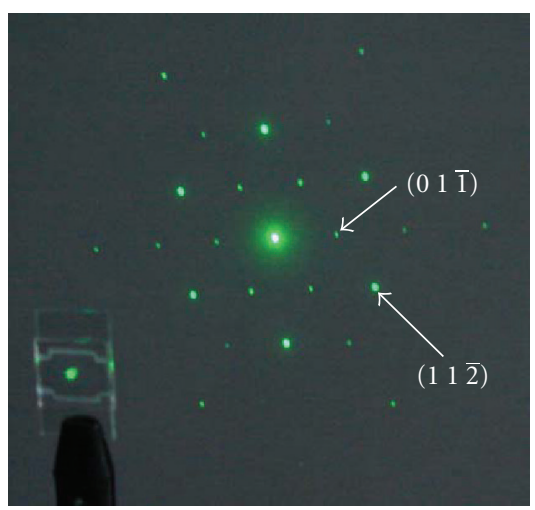

(a)

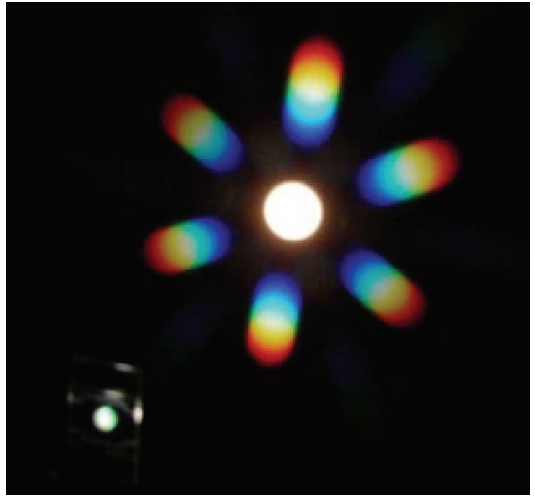

(b)

FigURE 58: Visible diffraction patterns of the H-PDLC PhCs produced by (a) a normally incident He-Ne laser beam and (b) a collimated broadband white beam.

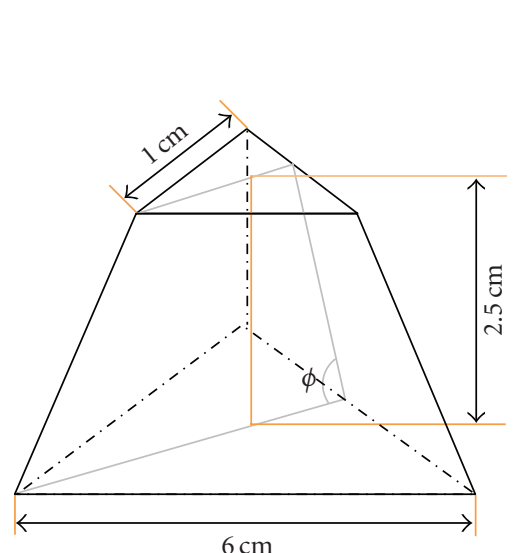

(a)

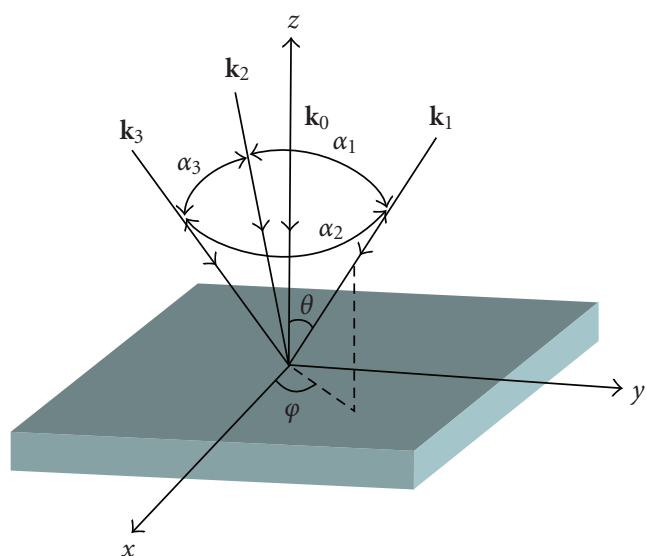

(b)

FIgURE 59: (a) Schematic of the specially designed prism and (b) four-beam interference configuration. 


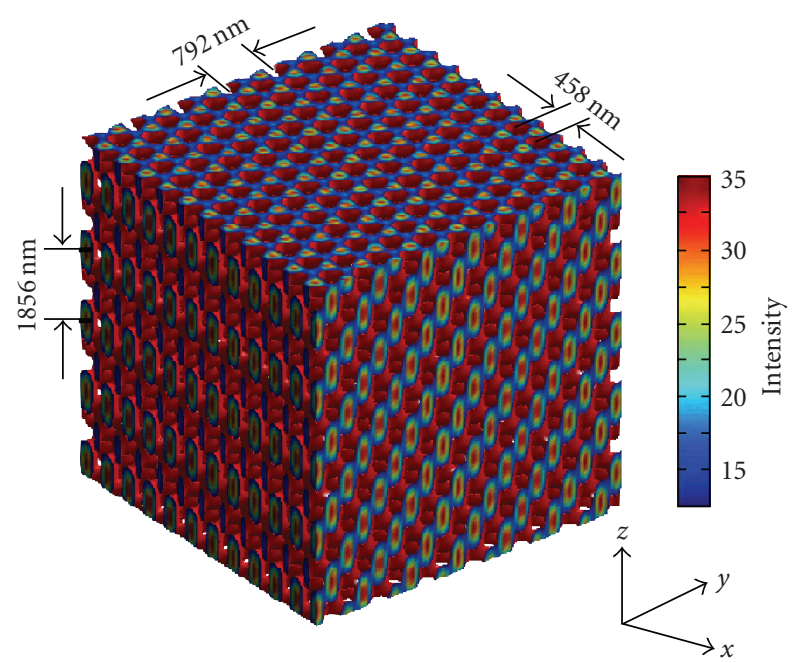

FIGURE 60: Simulated 3D interference pattern. The color bar shows the light intensity distribution.

used. Hsiao et al. reported the lasing emission from dyedoped H-PDLC transmission grating [135]. Compared to the reflection mode, the transmission mode grating offers a longer gain length without increasing the film thickness.

We systematically investigated the single-mode lasing properties from the dye-doped H-PDLC transmission gratings and showed much improved lasing properties including lower threshold, narrower linewidth, and higher Q factor, as shown in Figure 66. We also found that LC concentration played an important role in the lasing generation and wavelength selection. In our experiments, an LC concentration of $35 \mathrm{wt} \%$ was an approximately critical value to obtain a single-mode lasing from dye-doped $\mathrm{H}$ PDLC. With the decrease of the LC concentration, the lasing wavelength was blue shifted. Above this concentration, only amplified spontaneous emission (ASE) was observed. Typically, a $30 \mathrm{wt} \%$ LC concentration was used for H-PDLC transmission gratings to achieve high diffraction efficiency. However, for lasing generation, the LC concentration of around $20 \mathrm{wt} \%$ was found to be the best tradeoff between sufficient index modulation and optical scattering in the samples. We changed the LC and TMPTA concentrations and kept the other material concentrations fixed. The detailed weight percentage of each component used in the material syrup is shown in Table 3.

Figure 67 shows the normalized lasing spectra for $\mathrm{H}$ PDLC samples with various LC concentrations excited by about two times of the corresponding threshold. It can be seen from Figure 67 that, the linewidth of the lasing peak becomes narrower with the decrease of the LC concentration. The full width at half maximum (FWHM) of the lasing peak centering at $584 \mathrm{~nm}$ was less than $0.8 \mathrm{~nm}$. The transmission curves along the grating vector direction are also theoretically calculated, as shown in Figure 67. The refractive index of polymer matrices was assumed to be 1.54 . The other data used in our calculations were tabulated in Table 4. It is worth mentioning that in our calculations, the wavelength
TABLE 3: wt $\%$ of each component used in the prepolymer syrup for samples $1-6$.

\begin{tabular}{lccccccc}
\hline No. & TMPTA & NVP & NPG & RB & OA & DCM & E7 \\
\hline 1 & 67.1 & 7.8 & 0.9 & 0.6 & 8.0 & 1.0 & 14.6 \\
2 & 63.1 & 5.6 & 0.7 & 0.5 & 9.2 & 1.2 & 19.7 \\
3 & 57.8 & 6.0 & 0.7 & 0.5 & 9.5 & 1.2 & 24.3 \\
4 & 53.6 & 6.5 & 0.8 & 0.5 & 7.4 & 1.4 & 29.8 \\
5 & 47.6 & 7.4 & 0.9 & 0.6 & 8.1 & 1.2 & 34.2 \\
6 & 34.1 & 6.3 & 0.7 & 0.6 & 8.4 & 1.1 & 48.8 \\
\hline
\end{tabular}

TABle 4: Data used for the transmission curve calculation. $\Lambda_{G}$ is the grating pitch, $V_{\mathrm{LC}}: V_{\mathrm{p}}$ is the volume ratio of LC-rich lamellae to polymer-rich lamellae, respectively, $\Delta n_{1}$ is the index modulation.

\begin{tabular}{lccc}
\hline No. & $\Lambda_{G}(\mu \mathrm{m})$ & $V_{\mathrm{LC}}: V_{\mathrm{p}}$ & $\Delta n_{1}$ \\
\hline 1 & 0.564 & $16: 84$ & 0.05 \\
2 & 0.572 & $25: 75$ & 0.07 \\
3 & 0.583 & $45: 55$ & 0.09 \\
4 & 0.584 & $50: 50$ & 0.10 \\
5 & 0.584 & $55: 45$ & 0.11 \\
\hline
\end{tabular}

dispersion of the refractive indices of LC and polymer used are not considered because the refractive index changes of LC [136] and polymer [137] are small enough to be ignored. It is clear that the lasing action happens at one edge of the reflection band. The inset in Figure 67 shows a typical emission photograph of the dye-doped H-PDLC, where a sharp lasing point can be clearly observed. The estimated divergence angle from the far-field pattern was $\sim 15 \mathrm{mrad}$ and $\sim 65 \mathrm{mrad}$ in the directions perpendicular and parallel to the substrates, respectively.

As reported previously, lasing properties of H-PDLC transmission gratings are strongly dependent on the morphology of the grating. In our previous report, the morphology significantly changed in H-PDLC transmission gratings as the LC concentration changed. At a higher concentration, the volume fraction of LC-rich lamella increases and the LC droplets become larger. As a result, a larger scattering loss will be induced and the lasing has a larger threshold and wider FWHM. At a lower concentration, the volume fraction of LCrich lamellae decreases and the LC droplets become smaller. Within the LC-rich lamellae, a large amount of polymer exists and the index modulation is lowered accordingly. As a result, only a higher threshold can sustain enough distributed feedback, and a narrower FWHM is obtained due to the smaller scattering loss. Moreover, lower LC concentration reduces the index modulation, resulting in a blue-shifted bandgap. Therefore, the lasing wavelength is blue shifted accordingly.

For the devices based on H-PDLC, a distinct advantage is that they can be tuned or switched by an external stimulus. Hsiao et al. reported the electrically switchable lasing emission from the dye-doped H-PDLC transmission gratings [135]. Besides the electric method, the lasing can 


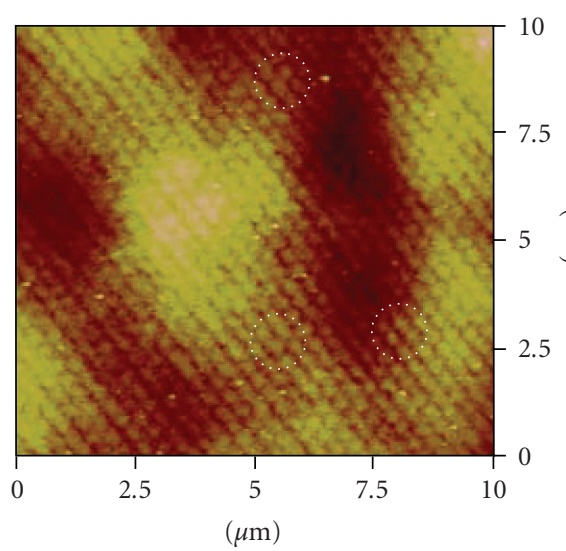

(a)

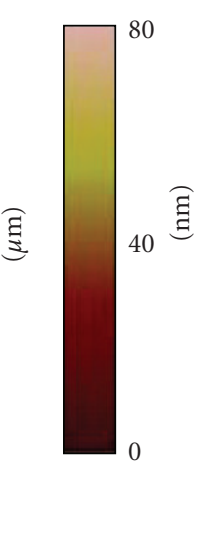

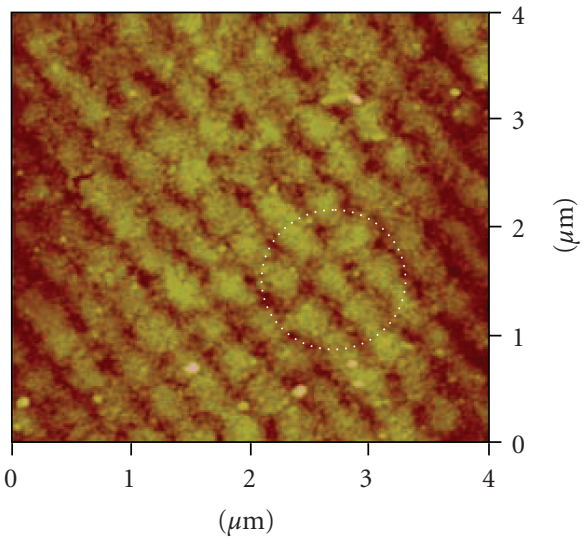

(b)

Figure 61: Surface AFM images of the 3D H-PDLC PhC sample in area of (a) $10 \times 10 \mu \mathrm{m}^{2}$ and (b) $4 \times 4 \mu \mathrm{m}^{2}$. The hexagonal structures are indicated in the dashed circles.

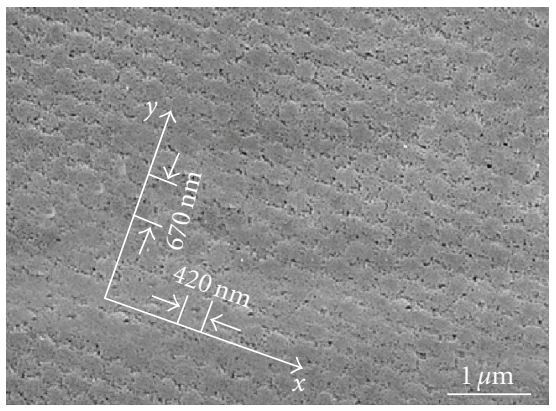

(a)

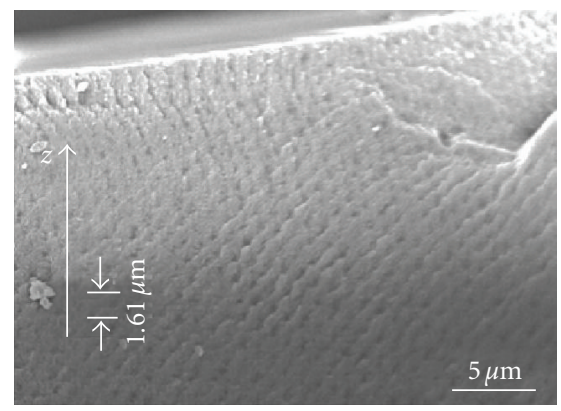

(b)

Figure 62: (a) SEM images of the surface and (b) cross-section of 3D H-PDLC PhC sample.

be also thermally switched. In our experiments, during the lasing measurement, a heater is used to control the temperature of the samples. The controllable temperature ranges from 20 to $80^{\circ} \mathrm{C}$, with an accuracy of about $3^{\circ} \mathrm{C}$.

Figure 68 shows the emission spectra from dye-doped H-PDLC gratings used no. 1 material syrup at various temperatures with a fixed pumping energy of $40 \mu \mathrm{J} /$ pulse. From Figure 68, we can see that the lasing can be thermally switched. It is interesting to note that at a temperature of $30^{\circ} \mathrm{C}$, another lasing peak centered at $595 \mathrm{~nm}$ appears in the emission envelope, as shown in Figure 68, which means at this temperature a sufficient gain can also be obtained for $595 \mathrm{~nm}$. The switching mechanism of the H-PDLC grating is due to the index modulation of the phase separated LC droplets. Generally, H-PDLC gratings show anisotropic more or less because there is a known volume shrinkage during the grating formation [14, 138], which makes the LC droplets anisotropic. With the increase of the temperature, the optical anisotropy reduces and accordingly the effective refractive index of the LC changes and at some temperature, the effective refractive index of the LC matches closely with the refractive index of the polymer matrix. As a result, the lasing emission disappears. Unlike described in [42], where the lasing wavelength shifted around $5 \mathrm{~nm}$, in our experiments, the effective refractive index does not change so much that there is no shift in the lasing wavelength. From Figure 68, we can see that, at the temperature of $45^{\circ} \mathrm{C}$, both original and new appeared lasing peaks nearly disappear. After cooling down, the lasing emission appears again.

\subsection{Sensors Based on Reflective H-PDLCs}

$\mathrm{H}$-PDLC reflection gratings are Bragg gratings formed of alternating layers of liquid crystals and polymer matrix as shown in Figure 69. They reflect a certain wavelength of light determined by the Bragg's grating equation given by

$$
\Lambda=\frac{\lambda}{2 n_{\text {eff }} \sin \theta},
$$

where $\Lambda$ is the fringe spacing of the grating vector, $n$ is the effective refractive index of the polymer and liquid crystal composition, $\theta$ is the angle at which the light source is incident, and $\lambda$ is the wavelength of the reflected light. The reflected wavelength of light is proportional to the fringe spacing of the gratings as can be seen from (57). 


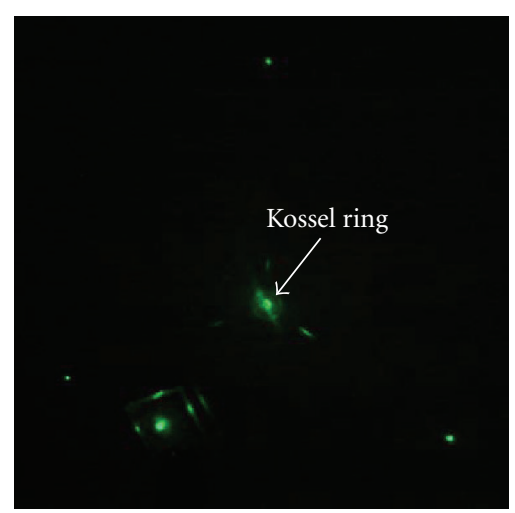

(a)

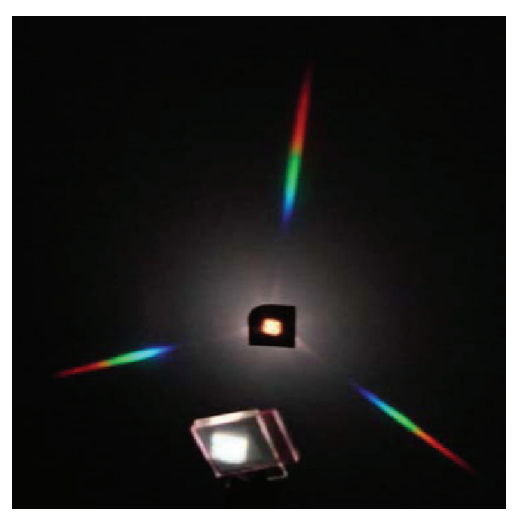

(b)

FIGURE 63: Diffraction patterns of the H-PDLC PhCs produced by (a) a normally incident He-Ne laser beam and (b) a broadband white beam.

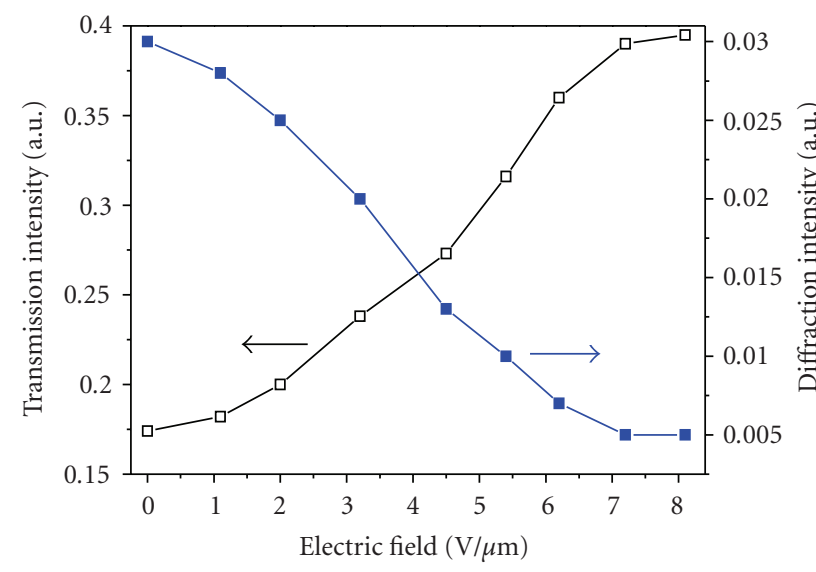

Figure 64: Diffraction and transmission intensities change as functions of applied voltage.

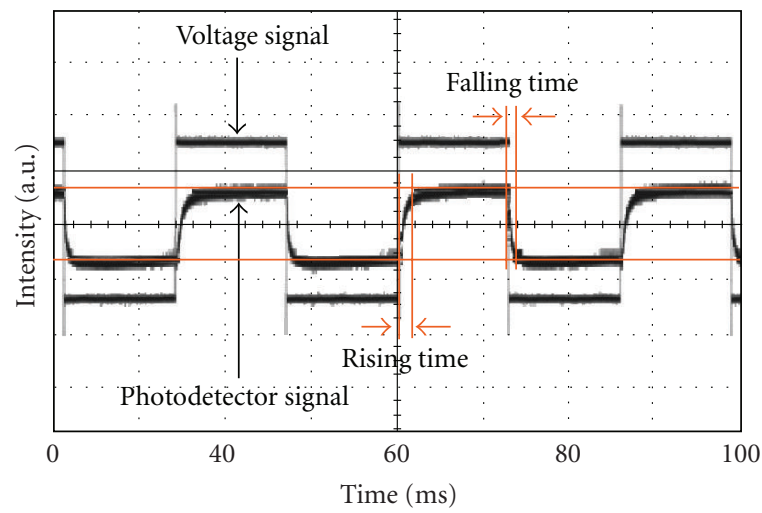

FIGURE 65: Electro-optical response time measurement of 3D HPDLC PhC sample.

Hence, theoretically decreasing the fringe spacing reduces the wavelength of the reflected light. On the other hand, if the fringe spacing is fixed, the reflected light can be shifted by changing the effective refractive index.

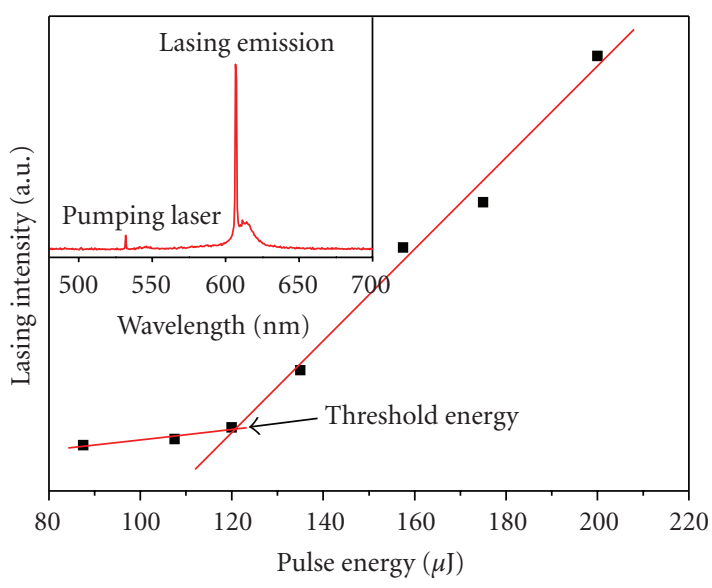

FIgURE 66: Output lasing intensity from DCM-doped H-PDLC transmission grating as a function of the laser pumping energy. The inset shows the lasing and the pump laser spectra.

\subsubsection{Pressure Sensor}

Based on the above concept, it can be applied to create an HPDLC pressure sensor. The fringe spacing is reduced by applying pressure perpendicular to the surface of the HPDLC and parallel to the grating vector. The wavelength reflected is measured for different applied pressure and the results are analyzed.

In one paper [140], the authors strained an H-PDLC reflection film perpendicular to the grating vector and observed a blue shift in the peak reflection wavelength of the film. This shift was caused by the Poisson contraction of the polymer segments of the grating parallel to the grating vector as the film was stretched perpendicular to the grating vector. Fontecchio et al. developed hydrostatic pressure sensors based on H-PDLC reflection gratings [139, 141]. Figure 70 shows the testing schematic. The light emitted from the fiber was collimated by a lens and spatially filtered. This light was then incident on the sensor under test at an angle of $\sim 30^{\circ}$ relative to the sensor normal. The light 


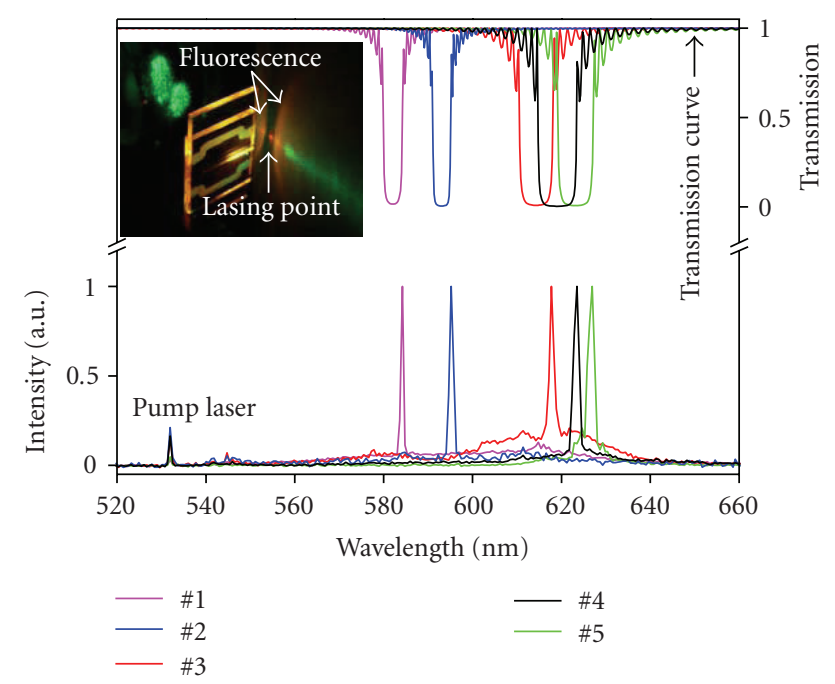

Figure 67: Normalized lasing spectra for H-PDLC samples with various LC concentrations excited by about two times of corresponding threshold. The inset shows a typical emission photograph of the dye-doped H-PDLC.

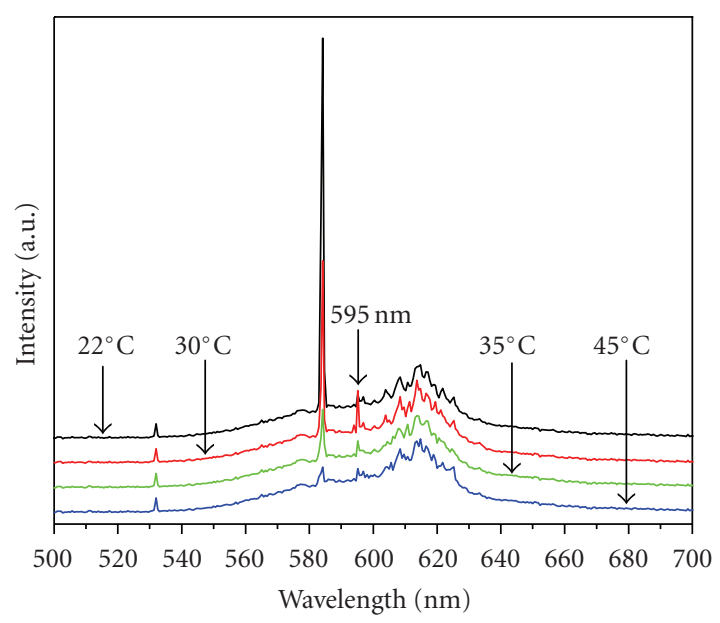

FIGURE 68: The emission spectra from dye-doped H-PDLC gratings at different temperatures.

reflected from the sensor was focused onto a fiber, which was connected to spectrometer with a high resolution. Changing the material formation can greatly improve the sensitivity of H-PDLC pressure sensors. The experimental results show that increasing the initiator concentration makes the $\mathrm{H}$ PDLC more elastic. Figure 71 shows the response for the difunctional polymer with various initiator concentrations. A clear difference can be observed from Figure 71 that the largest wavelength shift was obtained at the initiator concentration of $21 \mathrm{wt} \%$.

\subsubsection{Chemical Sensor}

With special treatment, the reflective H-PDLCs can contain many air voids without any damage to the grating structures. Therefore, the Bragg reflection notch of these gratings can

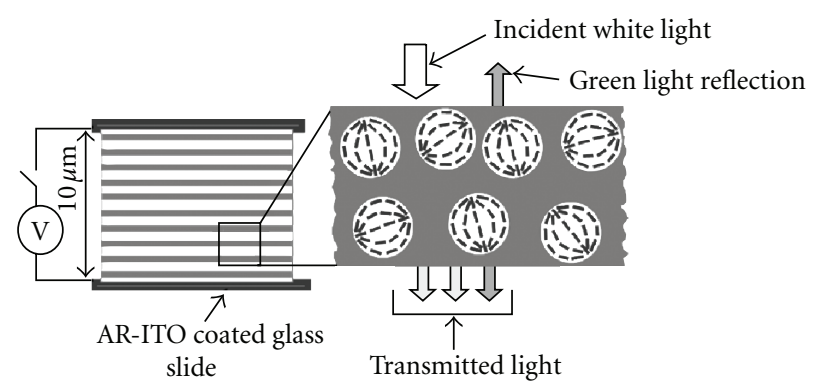

Figure 69: Illustration of reflective H-PDLCs. The LC droplets and polymer matrix are seen in alternating layers [139].

then be shifted by filling the voids inside the grating structure with solvent vapor. The high porosity of the grating film allows chemical vapors to easily permeate into the void regions, consequently the average refractive index is changed and the corresponding reflective wavelength shifts according to (57). The shifted reflective wavelength can be observed easily by eye due to the high refractive index contrast of the grating films. Thus, these holographic porous polymer gratings can act as colorimetric vapor sensors.

An easy way to create the voids in H-PDLCs is to simply add a nonreactive solvent, such as acetone or toluene, into the prepolymer syrup prior to holographic exposure. After exposure, the porous acrylate polymer grating structures can be created when the nonreactive solvent evaporates upon exposing the polymerized film to air. Porous $\mathrm{H}$ PDLC reflection grating used to detect chemical vapor has been successfully demonstrated [142], where $20 \mathrm{wt} \%$ toluene was used as nonactive solvent. When this porous grating was exposed to the air containing various concentration of acetone vapor, different reflection bands were clearly observed, which was induced by the refractive index change, as shown in Figure 72. A typical cycling behavior of the porous grating subjected to alternating acetone vapor is shown in Figure 73.

The big advantages of optical sensors are faster, safer, and easier to implement compared to those electrical sensors [143]. The reflective H-PDLC gratings show themselves potentially useful in chemical or pressure sensing, although the long term stability is still problematic for the H-PDLC materials.

\subsection{Neutron-Optical Devices Based on H-PDLC}

Besides the applications listed above, it is worth mentioning a latest emerging application based on H-PDLCs. It is $\mathrm{H}-$ PDLC-based neutron-optical devices [144, 145]. H-PDLCs can act as extremely efficient gratings not only for light but also for neutrons. H-PDLCs show similar diffraction behavior for the neutrons like light diffraction, as shown in Figure 74. The results showed that the light-induced refractive-index modulation for neutrons is about $10^{-6}$, that is, nearly 2 orders of magnitude larger than in the best photoneutron-refractive materials probed up to now, which makes 


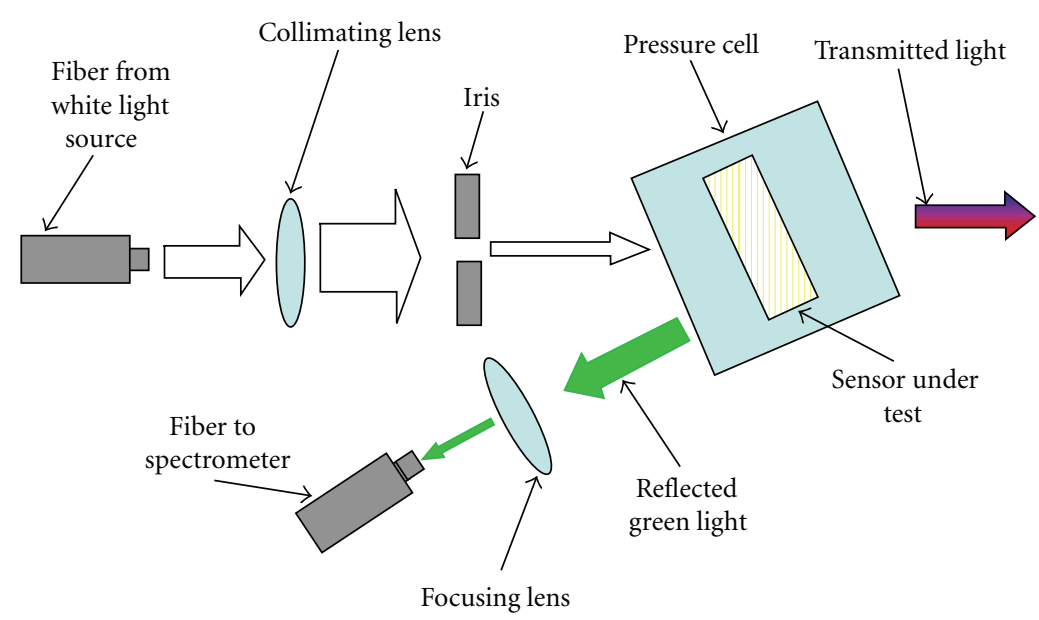

FIgURE 70: The configuration used to measure the reflected spectrum of the H-PDLC pressure sensors. Light is incident on the film at $\sim 30 \circ$ to the sensor normal [141].

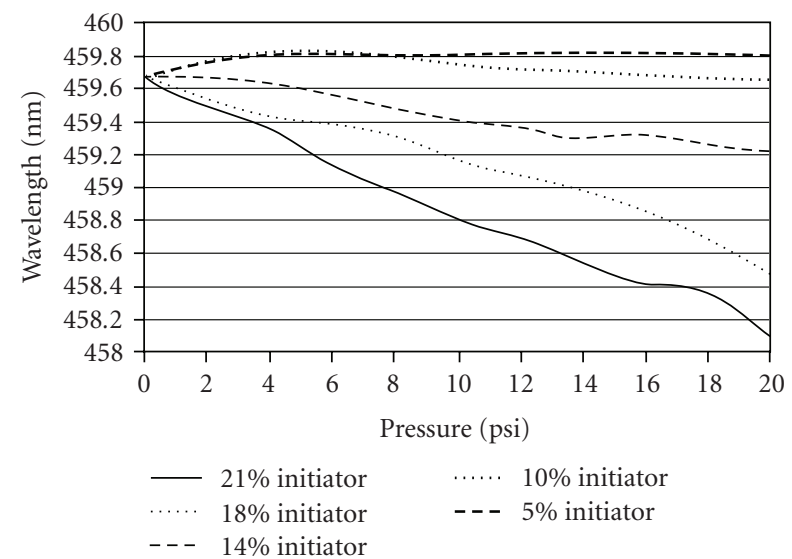

FIGURE 71: Wavelength versus pressure response of difunctional HPDLC with pressure applied from 0-20 psi [139].

H-PDLC promising as new materials to fabricate neutronoptical devices for cold and ultracold neutrons, such as beam splitters, lenses, and mirrors.

\section{Several Issues of H-PDLCs}

\subsection{Driving Voltage}

In H-PDLC, the LC droplets are so small (usually in the order of nanometers) that the surface area of interaction between polymer matrix and LC droplets is strongly enlarged, and hence the anchoring energy of LC droplets is greatly increased. Thus, a high driving voltage will be needed to reorientate the directors of $\mathrm{LC}$ molecules. This is the main reason why the driving voltage in H-PDLC gratings is higher than that in conventional PDLC devices. How to reduce the driving voltage is the key for H-PDLC applications. As a matter of fact, many factors affect the driving voltage of H-PDLCs, such as the ratios of components in the material formulation, morphologies of gratings, adding a

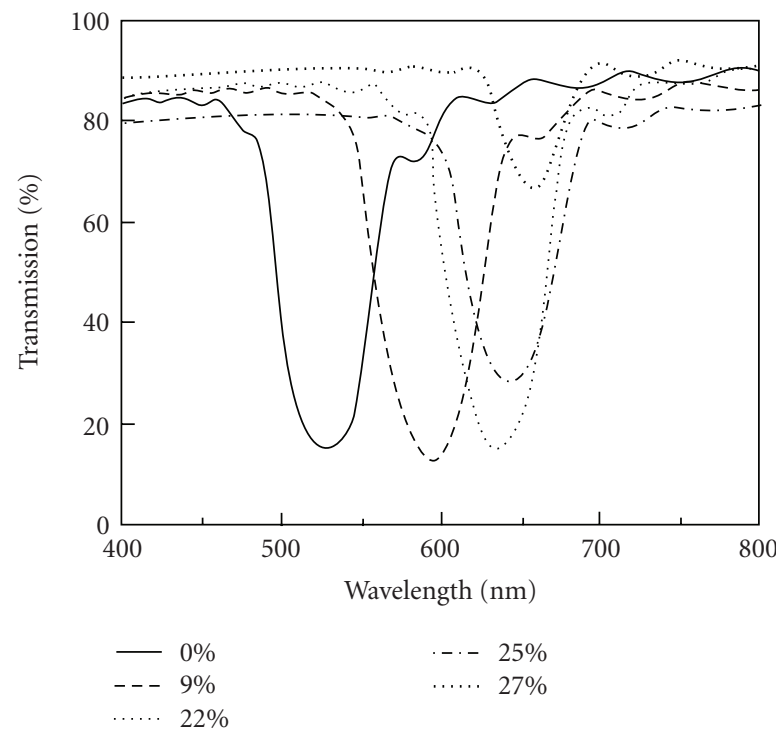

FIGURE 72: The transmission spectra of a typical grating film in air containing various concentrations of acetone vapor (volume-\%) relative to $1 \mathrm{~atm}(101.325 \mathrm{kPa})$ air pressure [142].

small amount of surfactant $[88,90,91]$ or high dielectric anisotropy material [87], and fluorination of polymer matrix [92, 93]. Many researchers studied the morphology effect on the driving voltages [71, 81, 146]. For example, with suitable LC weight ratio, two different grating morphologies in the LC-rich region, droplet-like (most H-PDLCs) and channel-like (POLYCRYPS gratings), can be formed, which greatly affect the driving voltage. Generally, the channellike H-PDLC gratings have a much lower driving voltage compared to the droplet-like ones. However, the dropletlike H-PDLCs usually show much faster response time compared to the channel-like ones. As a result, from the application view point, a good tradeoff between the driving voltage and response time is taken into account. On the other hand, adding a small portion of surfactant or partial 


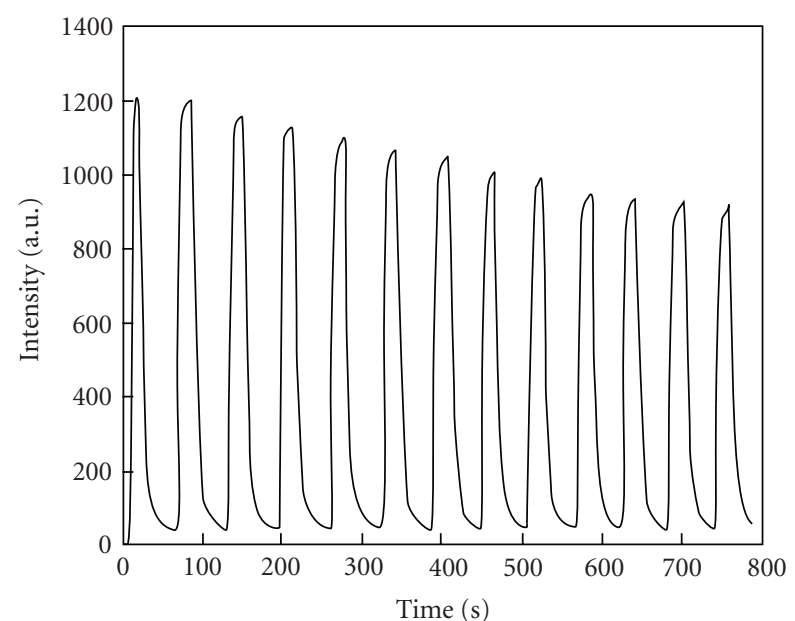

FIGURE 73: Cycling behavior of the porous grating subjected to alternating acetone vapor from 0.2 to $1 \mathrm{mbar}(0.02$ to $0.1 \mathrm{~Pa})$ [142].

fluorination of polymer may effectively reduce the driving voltage, as discussed in Section 7.5. However, adding another component into the prepolymer mixture will increase the complexity of material formulation as a kind of pollutant.

\subsection{Holographic Scattering}

The history of holographic scattering (HS) is as long as the history of the photorefractive effect. The first photograph of the HS phenomenon was found in 1966 when Ashkin et al. recognized the phenomenon of optical damage resulted from a light-induced refractive-index change [147]. Rather soon the photorefractive effect was utilized for the storage of volume phase holograms $[148,149]$, thus opening a new field for nonlinear optics.

The generic observation of HS is the following: if a laser beam propagates through a photorefractive crystal there is a gradual increase of light scattering with time, and thus a considerable decrease of the transmission. This kind of scattering shares two characteristic properties with volume holograms: it builds up with a time constant of the same order of magnitude characteristic for holographic recording and disappears immediately upon a slight rotation of the crystal or a change of the wavelength, just in the same way as the reconstructed wave fields of volume holograms do upon violation of Bragg's condition.

For H-PDLCs, most researchers focused on developing their applications. However, very little is known about optical imperfections in the H-PDLC structures, which is very important as they determine the limits of the H-PDLC applicability in any realistic device. One of them is the evolution of unintentional photo-induced light scattering, known as holographic scattering (HS) in photorefractive media. This effect emerges from an initial scattering of the incident beam (pump beam) from inhomogeneities within the sample. The interference of the incident and scattered light produces a spatial modulation of intensity that is transferred to weak refractive-index gratings. Subsequently,

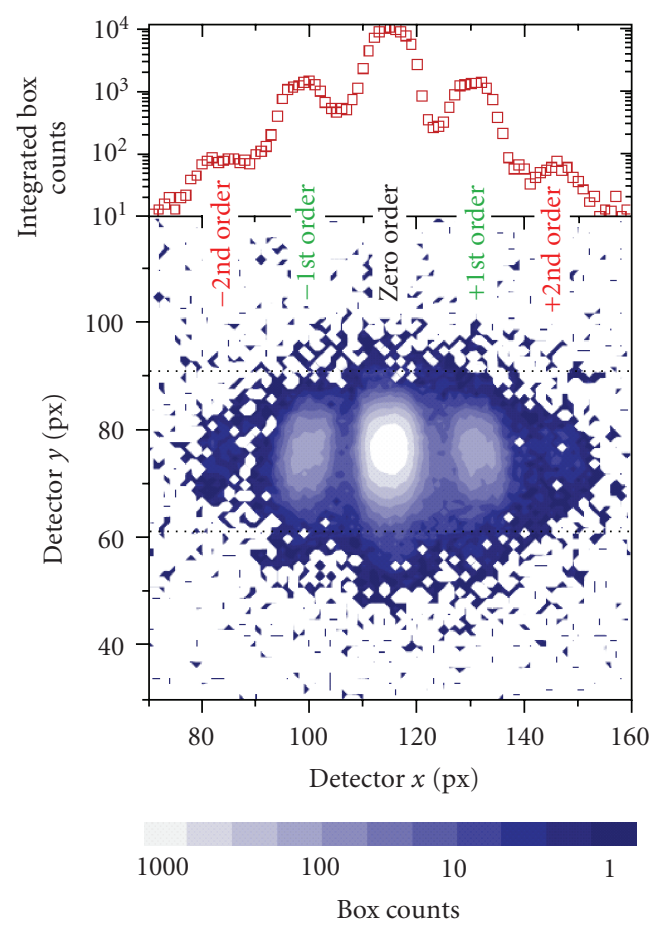

FIGURE 74: Diffraction pattern of an H-PDLC at a wavelength of $\lambda$ $=1.96 \mathrm{~nm}$. Diffraction up to the \pm second order is visible (bottom). The data points result from summing up the box counts of 30 pixels along the $y$-direction around the centers of diffraction spots, so that the second-order peaks are clearly visible (top) [144].

the pump beam is diffracted from these gratings. If the diffracted beam and the scattered beam are in phase, the latter will be amplified at the expense of the pump beam. This amplification process continues until certain limitations, that is, pump beam depletion, lead to a steady state. Finally, a multitude of parasitic holograms with different amplitudes and directions of grating vectors are recorded in the medium. As the parasitic holograms build up simultaneously with the desired primary hologram, they are also reconstructed at the same time. HS is a general phenomenon occurring in photosensitive materials.

Ellabban et al. studied the holographic scattering in $\mathrm{H}$ PDLCs $[150,151]$. Figure 75 shows the intensity distribution of the far-field scattered light monitored at different times of the UV exposure. A corona of scattered light develops spatially symmetric around the transmitted beam. Both the diameter and the intensity of the corona increase with time. Harbour et al. investigated the anisotropic scattering induced by the LC droplets during the formation of H-PDLC gratings [152], which showed that $p$-polarized light had a larger scattering than the $s$-polarized one.

Using an $\mathrm{He}-\mathrm{Ne}$ laser, the diffraction patterns at different angles of the probe beam were probed with respect to the sample normal. By rotating the sample, the symmetric corona of the scattered light transforms into two characteristic bright rings, as shown in Figure 76. The diameter of these rings changes with the readout angle and the wavelength. Forshaw explained these ring patterns are characteristic 

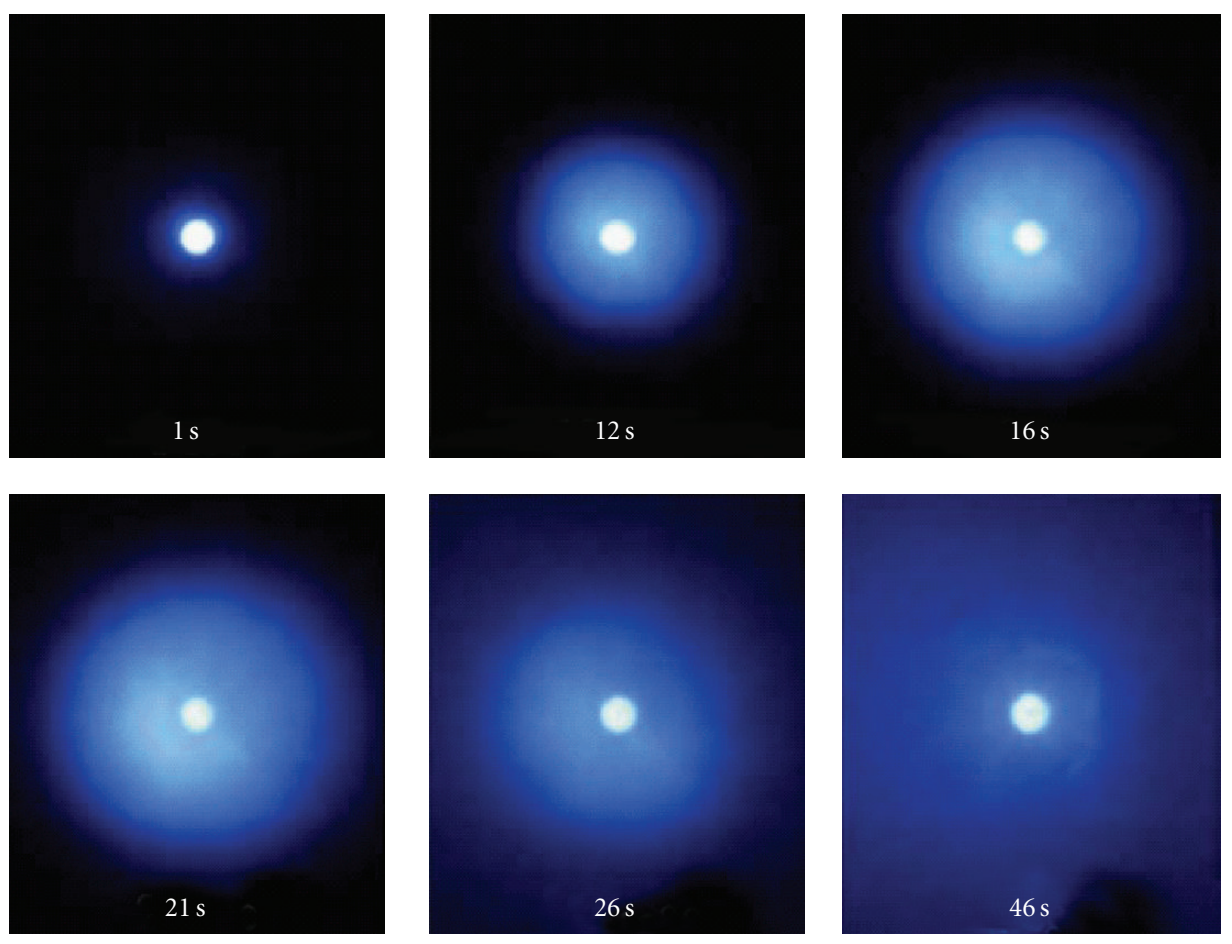

FIGURE 75: (Color online) Far-field scattering patterns observed at different recording times of the UV beam, $\lambda_{p}=351 \mathrm{~nm}$ and $I_{p}=$ $9 \mathrm{~mW} / \mathrm{cm}^{2}$. The central white spot corresponds to the transmitted beam [150].

for holographic scattering in terms of the Ewald sphere construction [153]. The intersection of the Ewald sphere (allowed wave vectors of propagation in the medium), with the structure factor (primary and conjugate sphere [153, $154]$ ), that is, the region in reciprocal space spanned by the recorded grating vectors, results in two cones. The projection of these cones on a screen then produces two bright rings. The apex angle of the cones derived from the Ewald-sphere construction is given by the following equation:

$$
\xi=2 \arctan \left[\frac{\sin \theta_{r}}{\cos \theta_{r} \pm \lambda_{p} / \lambda_{r}}\right]
$$

where $\theta_{r}$ is the readout angle measured with respect to the normal incidence of the readout beam within the medium. The positive sign is assigned to the conjugate sphere, the negative sign to the primary sphere.

In general, a big limitation of thick holographic recording media is connected with the origin of strong light-induced scattering during the process of optical recording. The presence of parasitic gratings (noise gratings) transfers almost all the light from the initial beam to the scattering directions and greatly corrupts the quality of any optical system. At the present, limited work has been done regarding the suppression of the HS for H-PDLCs.

\subsection{Polarization Dependence}

The polarization of the diffracted light depends on many factors, for example, monomers, liquid crystals, exposure conditions, and substrates. Karasawa and Taketomi once

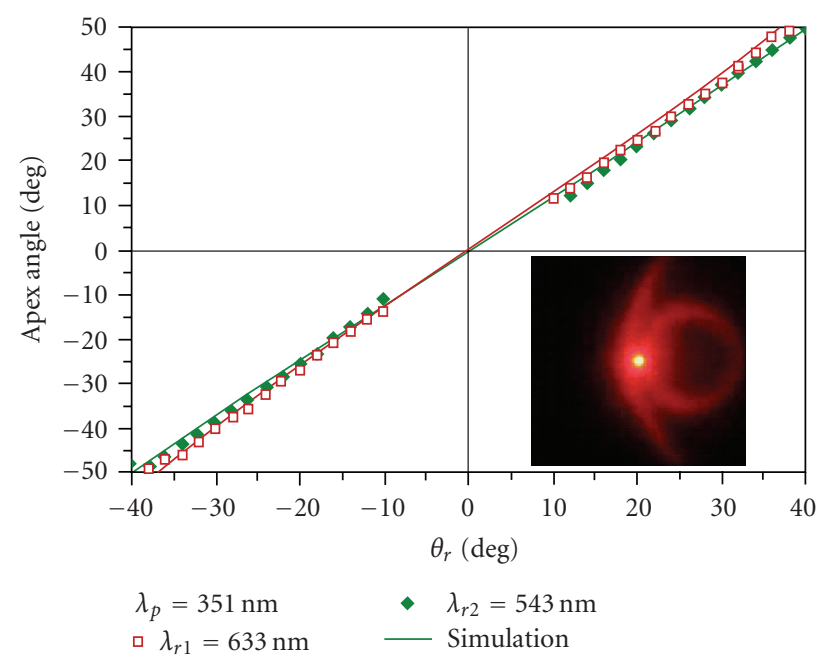

Figure 76: (Color online) Measured and calculated apex angle of the conjugate image versus readout angle for $\lambda_{r 1}=633$ and $\lambda_{r 2}=$ $543 \mathrm{~nm}$. Inset: far-field scattering pattern observed at $\theta_{r}=-14^{\circ}$ using $\lambda_{r 1}=633 \mathrm{~nm}$. The white spot corresponds to the transmitted beam [150].

investigated the effect of materials systems on the polarization behavior of H-PDLCs [155]. They showed the diffraction efficiency of the gratings strongly depended on the polarization of incoming light. Depending on the materials used, the diffraction properties are such that either $p$ - or $s$-polarized light is strongly diffracted while the light with 


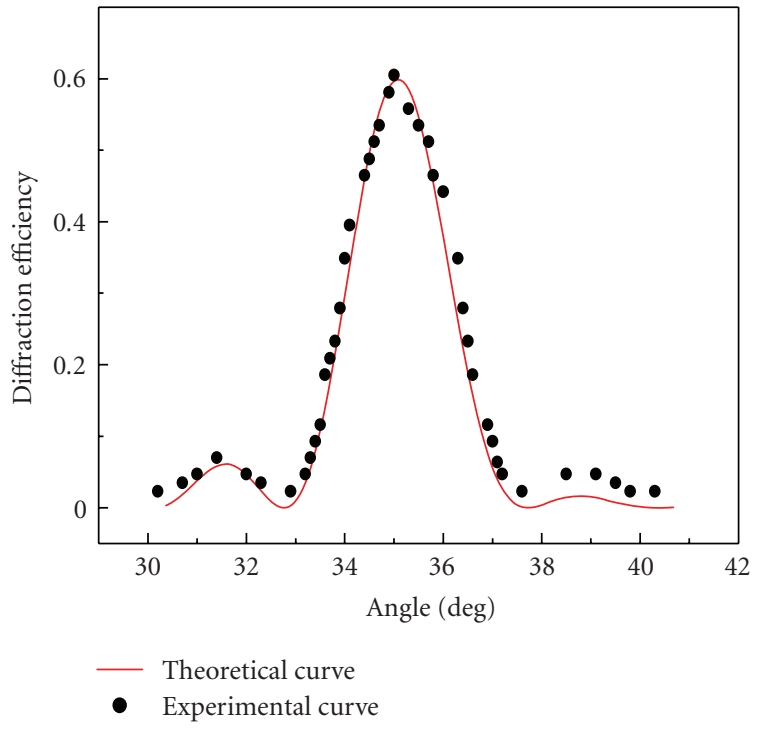

(a)

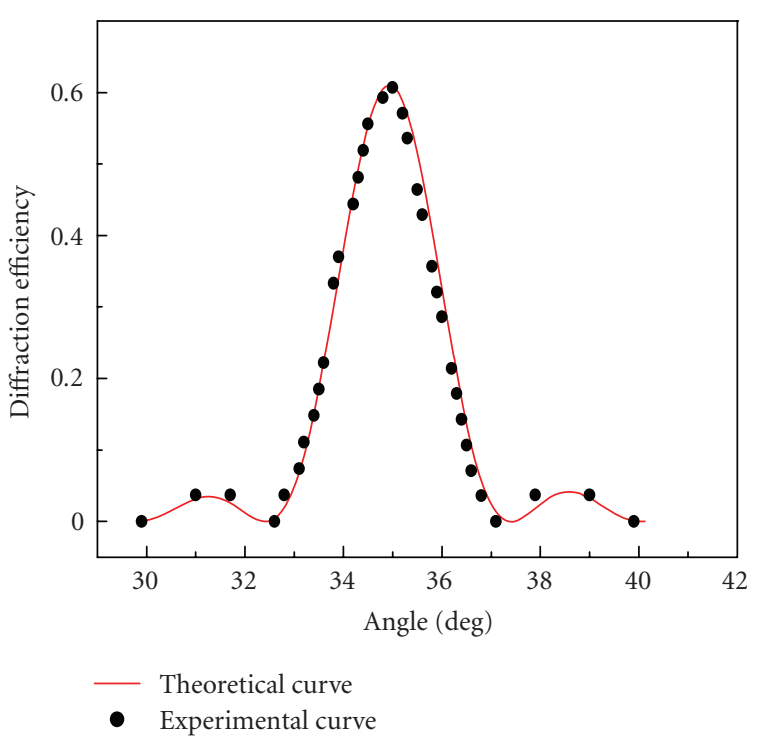

(b)

FIGURE 77: Diffraction efficiency as a function of incident angle for (a) $p$ - and (b) $s$-polarized light. The data is for an H-PDLC transmission grating with $\Lambda \approx 0.5 \mu \mathrm{m}$ probed at $632.8 \mathrm{~nm}$. The grating thickness is about $10 \mu \mathrm{m}$. A fit to coupled-wave theory yields (a) $n_{1}=0.054$ ( $p$-polarization) and (b) $n_{1}=0.019$ (s-polarization).

the other polarization is weakly diffracted. Sutherland et al. studied the polarization properties both theoretically and experimentally in detail $[73,74]$.

For LC-polymer composite optical switch, polarizationdependent loss (PDL) is a big concern. Generally, the conversion of the monomer molecules into a polymer network is accompanied with closer packing of molecules, which leads to contraction of the composite known as polymerization shrinkage. When the polymer shrinks, the LC droplets are compressed, which makes the symmetry axis of the rod-shape LC molecules in the droplet preferentially align along a common direction. This effect has been verified experimentally with in situ spectroscopy $[138,156]$. The shrinkage results in a polarization sensitive diffraction since the optical axis is on average along the same direction for all droplets, therefore making diffraction efficiency sensitive to the input polarization. However, in our experiment, the shrinkage was only about $2 \%$ which is significantly smaller than the reported data, which are usually $5 \% \sim 10 \%[48$, 157]. The smaller polymerization shrinkage reduces the PDL remarkably. The main reason for the lower shrinkage in our experiment is that, weaker exposure intensity and longer exposure time were used to decrease the shearing strength in the polymerization process. Figures $77(\mathrm{a})$ and $77(\mathrm{~b})$ show the diffraction efficiencies measured for $p$ - and $s$-polarized light for a wavelength of $632.8 \mathrm{~nm}$, respectively. Using the diffraction efficiency obtained in Figures 77(a) and 77(b), the PDL was calculated to be $0.03 \mathrm{~dB}$, by comparing the insertion loss difference between $p$ - and $s$-polarized light. It is worth mentioning that, Karasawa and Taketomi have reported that diffraction efficiency for $p$-polarization is much higher than that for $s$-polarization in acrylate systems [155], similar to that in Figure 78, which is also found in our experiments.
Figure 78 shows the diffraction efficiency for the sample fabricated in larger exposure intensity of $30 \mathrm{~mW} / \mathrm{cm}^{2}$. From Figure 78(a), it can be seen that, for $p$-polarization case, the left side lobe (more than 20\%) is much larger than the right one. This case is called overmodulation. The main reason is large shrinkage caused by intense exposure which makes the liquid crystals preferably aligned along one direction. From Figures 77 and 78, the angular bandwidth (full width at half maximum) is approximately $2^{\circ}$. According to the Kogelnik's coupled-wave theory [64], with increased thickness of LC-polymer composite films, the bandwidth will become narrower.

Smaller shrinkage is expected to reduce the shear force, thus decreasing the polarization dependence of H-PDLCs. Researchers have shown that small shrinkage could be obtained when a weak electric field was applied on the material film during the fabrication [158]. This is a good way to reduce the polarization dependence although the researchers didn't discuss it in their paper.

\subsection{Aging (Lifetime)}

The lifetime of H-PDLC is also a problem to be solved. Without any sealing, the performance of H-PDLC decays quickly after two or three months. Colegrove et al. studied the effect of the monomer functionality on the aging of acrylatebased H-PDLCs and found that the H-PDLCs formed with lower functionality monomers have better stability than those formed with higher functionality monomers [159]. For hexafunctional material, the LC droplets are connected and there is almost no polymer wall in the LC rich lamellae to support the polymer rich lamellae and substrates. LC 


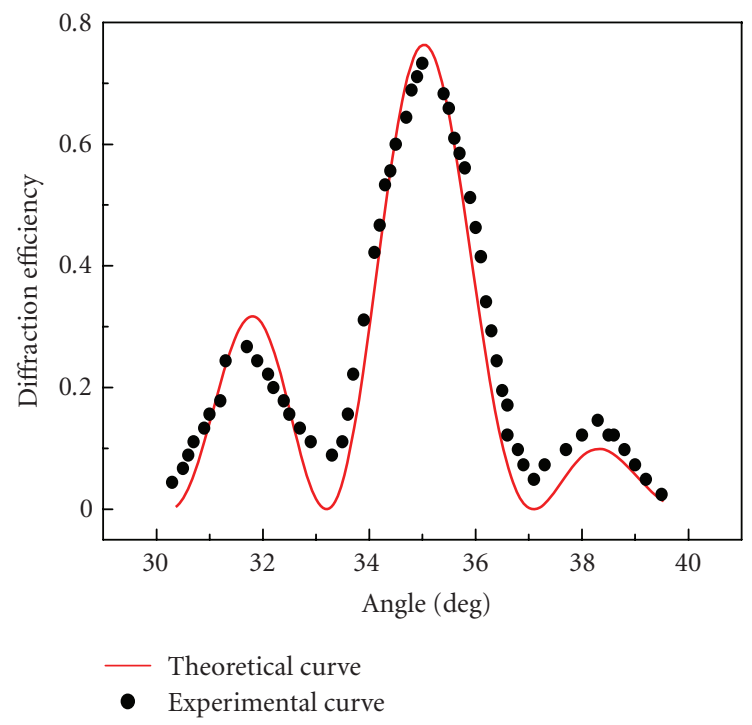

(a)

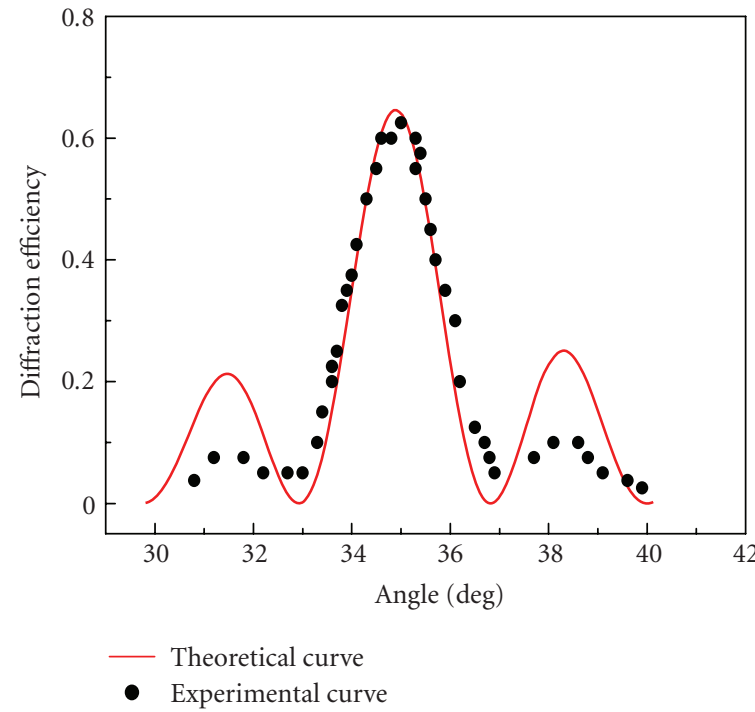

(b)

Figure 78: Diffraction efficiency as a function of incident angle for (a) $p$ - and (b) $s$-polarized light. The data is for a HPDLC transmission grating with $\Lambda \approx 0.5 \mu \mathrm{m}$ probed at $632.8 \mathrm{~nm}$. The grating thickness is about $10 \mu \mathrm{m}$. A fit to coupled-wave theory yields (a) $n_{1}=0.125$ ( $p$-polarization) and (b) $n_{1}=0.048$ (s-polarization). High side lobe indicates overmodulation.

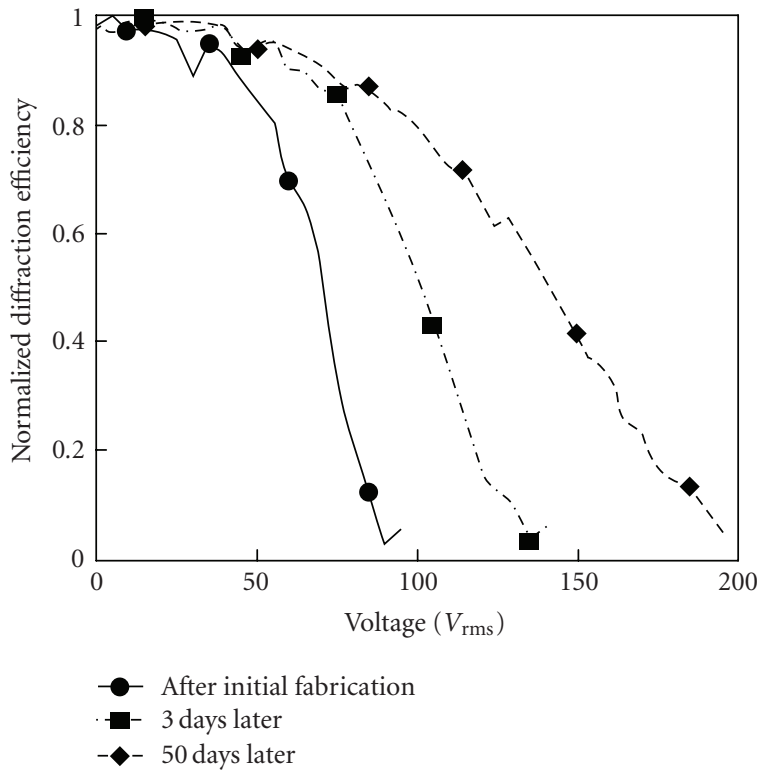

(a)

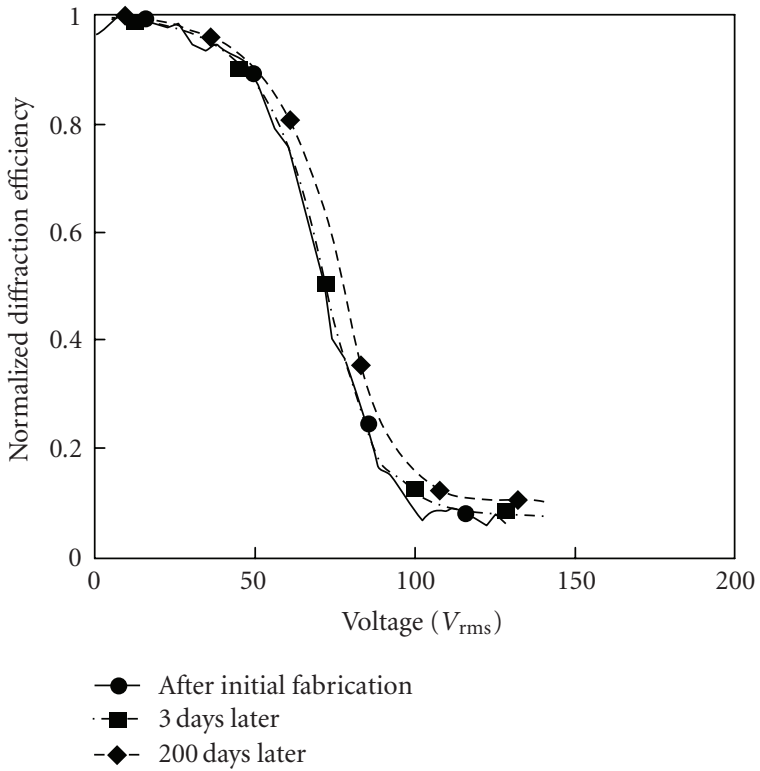

(b)

FIGURE 79: Electrical switching behavior (diffraction efficiency versus voltage) of (a) acrylate reflection gratings, and (b) thiol-ene reflection gratings with aging, respectively [48].

may slowly leak out and the performance of H-PDLCs then decayed much. For trifunctional material, the LC droplets are discrete and there are thicker polymer walls in the LC rich lamellae to support the polymer rich lamellae and substrates. There is no way for LC to leak out and the H-PDLCs show relatively long-term stability. Natarajan et al. compared the aging of reflection gratings based on acrylate and thiol-ene materials [48], respectively, as shown in Figure 79. For the much degraded acrylate-based H-PDLC, one possible reason is that the exposure is not enough. So after exposure, the materials can still continue to react with each other under the ambient light. Given only a 50\% conversion because of rapid vitrification, a substantial number of double bonds are present that can react slowly over time. The rapid gelation also traps a considerable amount of stress in the system. The fast gelation freezes a nonequilibrium network, which, over 
time, tries to equilibrate by stress relaxation via molecular motion. Such postillumination effects seem to be absent in thiol-ene gratings. The reason may be due to the much higher conversion obtained in the thiol-ene systems. Because all of the reactive double bonds are consumed, nothing exists to continue slow dark reactions, and the late gelation (at conversion approaching 100\%) eliminates large stresses. Another reason is that the acrylate materials themselves are not stable. They react with, for example, oxygen in air. The third reason is that, the materials operated under high electric field are vulnerable to be broken down. H-PDLC is a switchable device controlled by electric field. However, after repeating many times, the material properties may be altered.

\section{Summary and Remarks}

In summary, current status of H-PDLCs including materials, fabrication, performance optimization, applications, and existing issues has been reviewed. The H-PDLC technique has and will continue to create new applications. The ability to selectively control the diffraction properties of an optical element provides enormous freedom in the design and implementation of optical systems. Although impressive performance of H-PDLCs have been demonstrated to date, some fundamental issues are still not completely understood, especially in the process of the structure formation, in which polymerization, phase separation, and diffusion occur and interact with each other. Researchers have made much effort to overcome the issues existed in H-PDLCs, which pushes the $\mathrm{H}$-PDLCs to a practical application stage.

\section{Acknowledgment}

This project is supported by ICT Grant (Idea-Bank) under the Grant no. 2006ICTG03 of Nanyang Technological University, Singapore.

\section{References}

[1] S. L. Rosen, Fundamental Principles of Polymeric Materials, John Wiley \& Sons, New York, NY, USA, 1993.

[2] M. Hasegawa, "Response time improvement of the inplane-switching mode," in Proceedings of the International Symposium Digest of Technical Papers (SID '97), vol. 28, pp. 699-702, Santa Ana, Calif, USA, January-June 1997.

[3] M. J. Escuti, C. C. Bowley, G. P. Crawford, and S. Žumer, "Enhanced dynamic response of the in-plane switching liquid crystal display mode through polymer stabilization," Applied Physics Letters, vol. 75, no. 21, pp. 3264-3266, 1999.

[4] R. A. M. Hikmet, "Electrically induced light scattering from anisotropic gels," Journal of Applied Physics, vol. 68, no. 9, pp. 4406-4412, 1990.

[5] R. A. M. Hikmet, "Electrically induced light scattering from anisotropic gels with negative dielectric anisotropy," Molecular Crystals and Liquid Crystals, vol. 213, pp. 117-131, 1992.

[6] H. Ren and S.-T. Wu, "Anisotropic liquid crystal gels for switchable polarizers and displays," Applied Physics Letters, vol. 81, no. 8, pp. 1432-1434, 2002.
[7] F. Du and S.-T. Wu, "Curing temperature effects on liquid crystal gels," Applied Physics Letters, vol. 83, no. 7, pp. 13101312, 2003.

[8] J. L. Ferguson, "Polymer encapsulated nematic liquid crystals for display and light control applications," in Proceedings of the International Symposium Digest of Technical Papers (SID '85), vol. 16, pp. 68-70, San Jose, Calif, USA, 1985.

[9] J. W. Doane, N. A. Vaz, B.-G. Wu, and S. Žumer, "Field controlled light scattering from nematic microdroplets," Applied Physics Letters, vol. 48, no. 4, pp. 269-271, 1986.

[10] R. L. Sutherland, V. P. Tondiglia, L. V. Natarajan, T. J. Bunning, and W. W. Adams, "Electrically switchable volume gratings in polymer-dispersed liquid crystals," Applied Physics Letters, vol. 64, no. 9, pp. 1074-1076, 1994.

[11] T. J. Bunning, L. V. Natarajan, V. P. Tondiglia, and R. L. Sutherland, "Holographic polymer-dispersed liquid crystals (H-PDLCs)," Annual Review of Materials Science, vol. 30, pp. 83-115, 2000.

[12] D. Gabor, “A new microscopic principle," Nature, vol. 161, no. 4098, pp. 777-778, 1948.

[13] D. Gabor, "Microscopy by reconstructed wavefronts," Proceedings of The Royal Society of London A, vol. 197, pp. 454487, 1949.

[14] R. L. Sutherland, L. V. Natarajan, V. P. Tondiglia, and T. J. Bunning, "Bragg gratings in an acrylate polymer consisting of periodic polymer-dispersed liquid-crystal planes," Chemistry of Materials, vol. 5, no. 10, pp. 1533-1538, 1993.

[15] R. Asquini, A. d'Alessandro, C. Gizzi, et al., "Optical characterization at wavelengths of $632.8 \mathrm{NM}$ and $15498 \mathrm{NM}$ of policryps switchable diffraction gratings," Molecular Crystals and Liquid Crystals, vol. 398, pp. 223-233, 2003.

[16] G. Abbate, A. Marino, and F. Vita, "Policryps characterization in the near infrared," Molecular Crystals and Liquid Crystals, vol. 398, pp. 269-280, 2003.

[17] A. d'Alessandro, R. Asquini, C. Gizzi, et al., "Electro-optic properties of switchable gratings made of polymer and nematic liquid-crystal slices," Optics Letters, vol. 29, no. 12, pp. 1405-1407, 2004.

[18] R. Caputo, L. De Sio, A. Veltri, C. Umeton, and A. V. Sukhov, "Development of a new kind of switchable holographic grating made of liquid-crystal films separated by slices of polymeric material," Optics Letters, vol. 29, no. 11, pp. 12611263, 2004.

[19] R. Asquini, J. D’Angelo, and A. d'Alessandro, “A switchable optical add-drop multiplexer using ion-exchange waveguides and a POLICRYPS grating overlayer," Molecular Crystals and Liquid Crystals, vol. 450, no. 1, pp. 203-214, 2006.

[20] R. Caputo, L. De Sio, A. Veltri, C. P. Umeton, and A. V. Sukhov, "POLICRYPS switchable holographic grating: a promising grating electro-optical pixel for high resolution display application," Journal of Display Technology, vol. 2, no. 1, pp. 38-51, 2006.

[21] E. Lueder, R. Buerkle, M. Muecke, R. Klette, R. Bunz, and T. Kallfass, "Flexible and bistable FLC and cholesteric displays on plastic substrates for mobile applications and smart cards," Journal of the Society for Information Display, vol. 7, no. 1, pp. 29-35, 1999.

[22] K. E. Maly, M. D. Wand, and R. P. Lemieux, "Bistable ferroelectric liquid crystal photoswitch triggered by a dithienylethene dopant," Journal of the American Chemical Society, vol. 124, no. 27, pp. 7898-7899, 2002. 
[23] R. Karapinar, M. O’Neill, and M. Hird, "Polymer dispersed ferroelectric liquid crystal films with high electro-optic quality," Journal of Physics D, vol. 35, no. 9, pp. 900-905, 2002.

[24] J. N. Eakin, G. P. Crawford, and M. D. Radcliffe, "Morphological studies of holographically formed polymer dispersed ferroelectric liquid crystals," Molecular Crystals and Liquid Crystals, vol. 429, pp. 277-287, 2005.

[25] J. N. Eakin, G. P. Crawford, and M. D. Radcliffe, "Morphological studies of holographically formed polymer dispersed ferroelectric liquid crystals using elevated temperature exposure," Molecular Crystals and Liquid Crystals, vol. 439, pp. 2331, 2005.

[26] S. J. Woltman, J. N. Eakin, G. P. Crawford, and S. Žumer, "Holographic diffraction gratings using polymer-dispersed ferroelectric liquid crystals," Optics Letters, vol. 31, no. 22, pp. 3273-3275, 2006.

[27] K. K. Vardanyan, J. Qi, J. N. Eakin, M. De Sarkar, and G. P. Crawford, "Polymer scaffolding model for holographic polymer-dispersed liquid crystals," Applied Physics Letters, vol. 81, no. 25, pp. 4736-4738, 2002.

[28] A. Y.-G. Fuh, C.-R. Lee, and T.-S. Mo, "Polarization holographic grating based on azo-dye-doped polymer-ball-type polymer-dispersed liquid crystals," Journal of the Optical Society of America B, vol. 19, no. 11, pp. 2590-2594, 2002.

[29] A. Y.-G. Fuh, C.-R. Lee, Y.-H. Ho, T.-S. Mo, and P.-M. Liu, "Study of a holographic grating based on dye-doped polymer-ball-type polymer-dispersed liquid crystal films," Japanese Journal of Applied Physics, vol. 40, no. 12, pp. 68686871, 2001.

[30] J. Y. Woo, E. H. Kim, B. K. Kim, and Y. H. Cho, "Morphology and switching of holographic gratings containing an azo dye," Liquid Crystals, vol. 34, no. 4, pp. 527-533, 2007.

[31] S. Žumer and S. Kralj, "Influence of $k_{24}$ on the structure of nematic liquid crystal droplets," Liquid Crystals, vol. 12, no. 4, pp. 613-624, 1992.

[32] S. Kralj and S. Žumer, "Fréedericksz transitions in supra- $\mu \mathrm{m}$ nematic droplets," Physical Review A, vol. 45, no. 4, pp. 24612470, 1992.

[33] R. J. Ondris-Crawford, G. P. Crawford, S. Žumer, and J. W. Doane, "Curvature-induced configuration transition in confined nematic liquid crystals," Physical Review Letters, vol. 70, no. 2, pp. 194-197, 1993.

[34] P. Drzaic, "A new director alignment for droplets of nematic liquid crystal with low bend-to-splay ratio," Molecular Crystals and Liquid Crystals, vol. 154, pp. 289-306, 1988.

[35] H. Yuan, J. Colegrove, G. Hu, et al., "HPDLC color reflective displays," in Cockpit Dispalys VI: Displays for Defence Applications, vol. 3690 of Proceedings of SPIE, pp. 196-206, Orlando, Fla, USA, April 1999.

[36] G. P. Crawford, "Electrically switchable Bragg gratings," Optics \& Photonics News, no. 4, pp. 54-59, 142003.

[37] Y. Liu, B. Zhang, Y. Jia, and K. Xu, "Improvement of the diffraction properties in holographic polymer dispersed liquid crystal Bragg gratings," Optics Communications, vol. 218, no. 1-3, pp. 27-32, 2003.

[38] Y. J. Liu, X. W. Sun, J. H. Liu, H. T. Dai, and K. S. Xu, "A polarization insensitive $2 \times 2$ optical switch fabricated by liquid crystal-polymer composites," Applied Physics Letters, vol. 86, no. 4, Article ID 041115, 3 pages, 2005.
[39] V. P. Tondiglia, L. V. Natarajan, R. L. Sutherland, D. Tomlin, and T. J. Bunning, "Holographic formation of electrooptical polymer-liquid crystal photonic crystals," Advanced Materials, vol. 14, no. 3, pp. 187-191, 2002.

[40] Y. J. Liu and X. W. Sun, "Electrically tunable two-dimensional holographic photonic crystal fabricated by a single diffractive element," Applied Physics Letters, vol. 89, no. 17, Article ID 171101, 3 pages, 2006.

[41] S. P. Gorkhali, J. Qi, and G. P. Crawford, "Switchable quasi-crystal structures with five-, seven-, and ninefold symmetries," Journal of the Optical Society of America B, vol. 23, no. 1, pp. 149-158, 2006.

[42] R. Jakubiak, V. P. Tondiglia, L. V. Natarajan, et al., "Dynamic lasing from all-organic two-dimensional photonic crystals," Advanced Materials, vol. 17, no. 23, pp. 2807-2811, 2005.

[43] Y. J. Liu, X. W. Sun, P. Shum, et al., "Low-threshold and narrow-linewidth lasing from dye-doped holographic polymer-dispersed liquid crystal transmission gratings," Applied Physics Letters, vol. 88, no. 6, Article ID 061107, 3 pages, 2006.

[44] Y. J. Liu, X. W. Sun, H. I. Elim, and W. Ji, "Effect of liquid crystal concentration on the lasing properties of dye-doped holographic polymer-dispersed liquid crystal transmission gratings," Applied Physics Letters, vol. 90, no. 1, Article ID 011109, 3 pages, 2007.

[45] Y. J. Liu, X. W. Sun, H. I. Elim, and W. Ji, "Gain narrowing and random lasing from dye-doped polymer-dispersed liquid crystals with nanoscale liquid crystal droplets," Applied Physics Letters, vol. 89, no. 1, Article ID 011111, 3 pages, 2006.

[46] H. Ren, Y.-H. Fan, and S.-T. Wu, “Tunable Fresnel lens using nanoscale polymer-dispersed liquid crystals," Applied Physics Letters, vol. 83, no. 8, pp. 1515-1517, 2003.

[47] H. Ren, Y.-H. Fan, Y.-H. Lin, and S.-T. Wu, "Tunable-focus microlens arrays using nanosized polymer-dispersed liquid crystal droplets," Optics Communications, vol. 247, no. 1-3, pp. 101-106, 2005.

[48] L. V. Natarajan, C. K. Shepherd, D. M. Brandelik, et al., "Switchable holographic polymer-dispersed liquid crystal reflection gratings based on thiol-ene photopolymerization," Chemistry of Materials, vol. 15, no. 12, pp. 2477-2484, 2003.

[49] S.-T. Wu and A. Y.-G. Fuh, "Lasing in photonic crystals based on dye-doped holographic polymer-dispersed liquid crystal reflection gratings," Japanese Journal of Applied Physics, vol. 44, no. 2, pp. 977-980, 2005.

[50] D. C. Neckers, "Rose Bengal," Journal of Photochemistry and Photobiology A, vol. 47, no. 1, pp. 1-29, 1989.

[51] R. A. Ramsey and S. C. Sharma, "Switchable holographic gratings formed in polymer-dispersed liquid-crystal cells by use of a He-Ne laser," Optics Letters, vol. 30, no. 6, pp. 592594, 2005.

[52] R. A. Ramsey, S. C. Sharma, and K. Vaghela, "Holographically formed Bragg reflection gratings recorded in polymerdispersed liquid crystal cells using a He-Ne laser," Applied Physics Letters, vol. 88, no. 5, Article ID 051121, 3 pages, 2006.

[53] P. Pilot, Y. Boiko, and T. V. Galstian, "Near-IR (800 to $855 \mathrm{~nm}$ ) sensitive holographic photopolymer dispersed liquid crystal materials," in Liquid Crystal Materials, Devices, and Applications VII, vol. 3635 of Proceedings of SPIE, pp. 143-150, San Jose, Calif, USA, January 1999.

[54] K. Studer, C. Decker, E. Beck, and R. Schwalm, "Overcoming oxygen inhibition in UV-curing of acrylate coatings by carbon dioxide inerting-part I," Progress in Organic Coatings, vol. 48, no. 1, pp. 92-100, 2003. 
[55] K. Studer, C. Decker, E. Beck, and R. Schwalm, "Overcoming oxygen inhibition in UV-curing of acrylate coatings by carbon dioxide inerting-part II," Progress in Organic Coatings, vol. 48, no. 1, pp. 101-111, 2003.

[56] G. Zhao and P. Mouroulis, "Diffusion model of hologram formation in dry photopolymer materials," Journal of Modern Optics, vol. 41, no. 10, pp. 1929-1939, 1994.

[57] H. Oh, H. Lee, E. Kim, D. D. Do, and N. Kim, "Diffusion model of monomers in a photopolymer film for holographic recording," in Organic Holographic Materials and Applications IV, vol. 6335 of Proceedings of SPIE, pp. 1-8, San Diego, Calif, USA, August 2006.

[58] C. C. Bowley and G. P. Crawford, "Diffusion kinetics of formation of holographic polymer-dispersed liquid crystal display materials," Applied Physics Letters, vol. 76, no. 16, pp. 2235-2237, 2000.

[59] J. Qi, L. Li, M. De Sarkar, and G. P. Crawford, "Nonlocal photopolymerization effect in the formation of reflective holographic polymer-dispersed liquid crystals," Journal of Applied Physics, vol. 96, no. 5, pp. 2443-2450, 2004.

[60] A. Aslanyan and A. Galstyan, "Optimal period for diffraction gratings recorded in polymer dispersed liquid crystals," OptoElectronics Review, vol. 15, no. 1, pp. 66-70, 2007.

[61] T. Kyu, D. Nwabunma, and H.-W. Chiu, "Theoretical simulation of holographic polymer-dispersed liquid-crystal films via pattern photopolymerization-induced phase separation," Physical Review E, vol. 63, no. 6, Article ID 061802, 8 pages, 2001.

[62] T. K. Gaylord and M. G. Moharam, "Analysis and applications of optical diffraction by gratings," Proceedings of the IEEE, vol. 73, no. 5, pp. 894-937, 1985.

[63] M. Nevière and E. Popov, "Grating electromagnetic theory user guide," Journal of Imaging Science and Technology, vol. 41, no. 4, pp. 315-323, 1997.

[64] H. Kogelnik, "Coupled wave theory for thick hologram gratings," The Bell System Technical Journal, vol. 48, no. 9, pp. 2909-2947, 1969.

[65] M. Jazbinšek, I. Drevensek-Olenik, M. Zgonik, A. K. Fontecchio, and G. P. Crawford, "Characterization of holographic polymer dispersed liquid crystal transmission gratings," Journal of Applied Physics, vol. 90, no. 8, pp. 3831-3837, 2001.

[66] M. E. Holmes and M. S. Malcuit, "Controlling the anisotropy of holographic polymer-dispersed liquid-crystal gratings," Physical Review E, vol. 65, no. 6, Article ID 066603, 4 pages, 2002.

[67] J. J. Butler, M. S. Malcuit, and M. A. Rodriguez, "Diffractive properties of highly birefringent volume gratings: investigation," Journal of the Optical Society of America B, vol. 19, no. 2, pp. 183-189, 2002.

[68] D. E. Lucchetta, L. Criante, and F. Simoni, "Optical characterization of polymer dispersed liquid crystals for holographic recording," Journal of Applied Physics, vol. 93, no. 12, pp. 9669-9674, 2003.

[69] D. E. Lucchetta, L. Criante, and F. Simoni, "Determination of small anisotropy of holographic phase gratings," Optics Letters, vol. 28, no. 9, pp. 725-727, 2003.

[70] R. L. Sutherland, L. V. Natarajan, T. J. Bunning, and V. P. Tondiglia, "Switchable holographic polymer-dispersed liquid crystals," in Handbook of Advanced Electronic and Photonic Materials and Devices, H. S. Nalwa, Ed., vol. 7, chapter 2, Academic Press, San Diego, Calif, USA, 2001.

[71] R. L. Sutherland, L. V. Natarajan, V. P. Tondiglia, T. J. Bunning, B. L. Epling, and D. M. Brandelik, "Relation of electro-optical characteristics to materials properties and morphology in polymer-dispersed liquid crystal holographic gratings," in Diffractive and Holographic Device Technologies and Applications IV, vol. 3010 of Proceedings of SPIE, pp. 142149, San Jose, Calif, USA, February 1997.

[72] F. Vita, D. E. Lucchetta, R. Castagna, O. Francescangeli, L. Criante, and F. Simoni, "Detailed investigation of highresolution reflection gratings through angular-selectivity measurements," Journal of the Optical Society of America B, vol. 24, no. 3, pp. 471-476, 2007.

[73] R. L. Sutherland, "Polarization and switching properties of holographic polymer-dispersed liquid-crystal gratings. I. Theoretical model," Journal of the Optical Society of America B, vol. 19, no. 12, pp. 2995-3003, 2002.

[74] R. L. Sutherland, L. V. Natarajan, V. P. Tondiglia, et al., "Polarization and switching properties of holographic polymer-dispersed liquid-crystal gratings. II. Experimental investigations," Journal of the Optical Society of America B, vol. 19, no. 12, pp. 3004-3012, 2002.

[75] F. Vita, A. Marino, V. Tkachenko, et al., "Visible and near-infrared characterization and modeling of nanosized holographic-polymer-dispersed liquid crystal gratings," Physical Review E, vol. 72, no. 1, Article ID 011702, 8 pages, 2005.

[76] T. J. Bunning, L. V. Natarajan, V. P. Tondiglia, R. L. Sutherland, R. Haaga, and W. W. Adams, "Effects of eliminating the chain extender and varying the grating periodicity on the morphology of holographically written Bragg gratings," in Liquid Crystal Materials, Devices, and Applications IV, vol. 2651 of Proceedings of SPIE, pp. 44-55, San Jose, Calif, USA, January 1996.

[77] B. K. Kim, Y. C. Jeon, C. O. Yoon, K. J. Kim, and Y. H. Cho, "Optimization of holographic PDLC for green," Molecular Crystals and Liquid Crystals, vol. 368, pp. 87-96, 2001.

[78] N. J. Crawford, M. D. Dadmun, T. J. Bunning, and L. V. Natarajan, "Time-resolved light scattering of the phase separation in polymer-dispersed liquid crystals formed by photo-polymerization induced phase separation," Polymer, vol. 47, no. 18, pp. 6311-6321, 2006.

[79] A. K. Fontecchio, C. C. Bowley, H. Yuan, and G. P. Crawford, "Effect of monomer functionality on performance of holographically-formed polymer dispersed liquid crystals," Molecular Crystals and Liquid Crystals, vol. 352, pp. 399-406, 2000.

[80] R. T. Pogue, L. V. Natarajan, S. A. Siwecki, V. P. Tondiglia, R. L. Sutherland, and T. J. Bunning, "Monomer functionality effects in the anisotropic phase separation of liquid crystals," Polymer, vol. 41, no. 2, pp. 733-741, 2000.

[81] M. De Sarkar, N. L. Gill, J. B. Whitehead, and G. P. Crawford, "Effect of monomer functionality on the morphology and performance of the holographic transmission gratings recorded on polymer dispersed liquid crystals," Macromolecules, vol. 36, no. 3, pp. 630-638, 2003.

[82] M. S. Park and B. K. Kim, "Transmission holographic gratings produced using networked polyurethane acrylates with various functionalities," Nanotechnology, vol. 17, no. 8, pp. 2012-2017, 2006.

[83] T. J. Bunning, L. V. Natarajan, V. P. Tondiglia, G. Dougherty, and R. L. Sutherland, "Morphology of anisotropic polymerdispersed liquid crystals and the effect of monomer functionality," Journal of Polymer Science Part B, vol. 35, no. 17, pp. 2825-2833, 1997.

[84] T. J. White, W. B. Liechty, L. V. Natarajan, V. P. Tondiglia, T. J. Bunning, and C. A. Guymon, "The influence of $N$-vinyl2-pyrrolidinone in polymerization of holographic polymer 
dispersed liquid crystals (HPDLCs)," Polymer, vol. 47, no. 7, pp. 2289-2298, 2006.

[85] P. Mormile, P. Musto, L. Petti, G. Ragosta, and P. Villano, "Electro-optical properties of a PDLC based on unsaturated polyester resin," Applied Physics B, vol. 70, no. 2, pp. 249-252, 2000.

[86] B. G. Wu, J. H. Erdman, and J. W. Doane, "Response times and voltages for PDLC light shutters," Liquid Crystals, vol. 5, no. 5, pp. 1453-1465, 1989.

[87] J. Colegrove, H. Yuan, S. T. Wu, J. R. Kelly, C. C. Bowley, and G. P. Crawford, "Drive-voltage reduction for HPDLC displays," in Proceedings of the 6th International Display Workshops, pp. 105-108, Sendai, Japan, December 1999.

[88] S. S. Patnaik and R. Pachter, "Anchoring characteristics and interfacial interactions in a polymer dispersed liquid crystal: a molecular dynamics study," Polymer, vol. 40, no. 23, pp. 6507-6519, 1999.

[89] C. C. Bowley, P. Kossyrev, S. Danworaphong, J. Colegrove, J. Kelly, T. Fiske, H. J. Yuan, and G. P. Crawford, "Improving the voltage response of holographically-formed polymer dispersed liquid crystals (H-PDLCs)," Molecular Crystals and Liquid Crystals, vol. 359, pp. 327-339, 2001.

[90] G. P. Crawford, R. J. Ondris-Crawford, J. W. Doane, and S. Žumer, "Systematic study of orientational wetting and anchoring at a liquid-crystal-surfactant interface," Physical Review E, vol. 53, no. 4, pp. 3647-3661, 1996.

[91] J. Y. Woo and B. K. Kim, "Surfactant effects on morphology and switching of holographic PDLCs based on polyurethane acrylates," ChemPhysChem, vol. 8, no. 1, pp. 175-180, 2007.

[92] M. D. Schulte, S. J. Clarson, L. V. Natarajan, C. A. Guymon, and T. J. Bunning, "Holographic polymer dispersed liquid crystals: effect of partial matrix fluorination on electrooptical and morphological properties," in Proceedings of the Materials Research Society Symposium, vol. 709, pp. 211-216, Boston, Mass, USA, November 2002.

[93] M. De Sarkar, J. Qi, and G. P. Crawford, "Influence of partial matrix fluorination on morphology and performance of HPDLC transmission gratings," Polymer, vol. 43, no. 26, pp. 7335-7344, 2002.

[94] R. Ulrich and R. Torge, "Measurement of thin film parameters with a prism coupler," Applied Optics, vol. 12, no. 12, pp. 2901-2908, 1973.

[95] L. L. Brott, R. R. Naik, D. J. Pikas, et al., "Ultrafast holographic nanopatterning of biocatalytically formed silica," Nature, vol. 413, no. 6853, pp. 291-293, 2001.

[96] Y. Tomita, N. Suzuki, and K. Chikama, "Holographic manipulation of nanoparticle distribution morphology in nanoparticle-dispersed photopolymers," Optics Letters, vol. 30, no. 8, pp. 839-841, 2005.

[97] Y. Tomita, K. Chikama, Y. Nohara, N. Suzuki, K. Furushima, and Y. Endoh, "Two-dimensional imaging of atomic distribution morphology created by holographically induced mass transfer of monomer molecules and nanoparticles in a silicananoparticle-dispersed photopolymer film," Optics Letters, vol. 31, no. 10, pp. 1402-1404, 2006.

[98] N. Suzuki, Y. Tomita, K. Ohmori, M. Hidaka, and K. Chikama, "Highly transparent $\mathrm{ZrO}_{2}$ nanoparticle-dispersed acrylate photopolymers for volume holographic recording," Optics Express, vol. 14, no. 26, pp. 12712-12719, 2006.

[99] W. S. Kim, Y.-C. Jeong, and J.-K. Park, "Nanoparticleinduced refractive index modulation of organic-inorganic hybrid photopolymer," Optics Express, vol. 14, no. 20, pp. 8967-8973, 2006.
[100] R. Jakubiak, D. P. Brown, F. Vatansever, et al., "Holographic photopolymerization for fabrication of electrically switchable inorganic-organic hybrid photonic structures," in Organic Photonic Materials and Devices V, vol. 4991 of Proceedings of SPIE, pp. 89-97, San Jose, Calif, USA, January 2003.

[101] E. H. Kim, J. Y. Woo, and B. K. Kim, "Nanosized-silicareinforced holographic polymer-dispersed liquid crystals," Macromolecular Rapid Communications, vol. 27, no. 7, pp. 553-557, 2006.

[102] H. Ren and S.-T. Wu, "Reflective reversed-mode polymer stabilized cholesteric texture light switches," Journal of Applied Physics, vol. 92, no. 2, p. 797, 2002.

[103] J. Ma, J. Song, Y.-G. Liu, S.-P. Ruan, and L. Xuan, "Holographic reversed-mode polymer-stabilized liquid crystal grating," Chinese Physics Letters, vol. 22, no. 1, pp. 103-106, 2005.

[104] K. Tanaka, K. Kato, S. Tsuru, and S. Sakai, "Holographically formed liquid-crystal/polymer device for reflective color display," Journal of the Society for Information Display, vol. 2, no. 1, pp. 37-40, 1994.

[105] C. Z. van Doorn, "Dynamic behavior of twisted nematic liquid-crystal layers in switched fields," Journal of Applied Physics, vol. 46, no. 9, pp. 3738-3745, 1975.

[106] D. W. Berreman, "Liquid-crystal twist cell dynamics with backflow," Journal of Applied Physics, vol. 46, no. 9, pp. 37463751, 1975.

[107] R. L. Sutherland, L. V. Natarajan, V. P. Tondiglia, T. J. Bunning, and W. W. Adams, "Switchable holograms in new photopolymer-liquid crystal composite materials," in Diffractive and Holographic Optics Technology II, vol. 2404 of Proceedings of SPIE, pp. 132-143, San Jose, Calif, USA, February 1995.

[108] V. P. Tondiglia, L. V. Natarajan, R. L. Sutherland, T. J. Bunning, and W. W. Adams, "Volume holographic image storage and electro-optical readout in a polymer-dispersed liquid-crystal film," Optics Letters, vol. 20, no. 11, pp. 13251327, 1995.

[109] S.-T. Wu and A.Y.-G. Fuh, "Electrical-frequency switchable multi-domain polymer dispersed liquid crystal Bragg mirror," Japanese Journal of Applied Physics, vol. 45, no. 9, pp. 7011-7016, 2006.

[110] L. V. Natarajan, V. P. Tondiglia, R. L. Sutherland, D. Tomlin, and T. J. Banning, "Electro-optical and morphological properties of bragg transmission gratings written in holographic polymer dispersed liquid crystals by thiol-ene photopolymerization," in Proceedings of the Materials Research Society Symposium, vol. 776, pp. 225-230, San Francisco, Calif, USA, April 2003.

[111] A. F. Senyurt, G. Warren, J. B. Whitehead Jr., and C. E. Hoyle, "Matrix physical structure effect on the electro-optic characteristics of thiol-ene based H-PDLC films," Polymer, vol. 47, no. 8, pp. 2741-2749, 2006.

[112] R. L. Sutherland, V. P. Tondiglia, L. V. Natarajan, S. Chandra, D. Tomlin, and T. J. Bunning, "Switchable orthorhombic F photonic crystals formed by holographic polymerizationinduced phase separation of liquid crystal," Optics Express, vol. 10, no. 20, pp. 1074-1082, 2002.

[113] H.-S. Kitzerow, "Dual-frequency addressable gratings based on polymer-dispersed liquid crystals," Molecular Crystals and Liquid Crystals, vol. 321, pp. 457-472, 1998. 
[114] M. J. Escuti, J. Qi, and G. P. Crawford, "Tunable facecentered-cubic photonic crystal formed in holographic polymer dispersed liquid crystals," Optics Letters, vol. 28, no. 7, pp. 522-524, 2003.

[115] M. J. Escuti, J. Qi, and G. P. Crawford, "Two-dimensional tunable photonic crystal formed in a liquid-crystal/polymer composite: threshold behavior and morphology," Applied Physics Letters, vol. 83, no. 7, pp. 1331-1333, 2003.

[116] G. Yandek, S. Meng, G. Sigalov, and T. Kyu, "Threedimensional switchable polymer photonic crystals via various optical wave interference techniques," Liquid Crystals, vol. 33, no. 7, pp. 775-788, 2006.

[117] T. Kyu, S. Meng, H. Duran, K. Nanjundiah, and G. R. Yandek, "Holographic polymer-dispersed liquid crystals and polymeric photonic crystals formed by holographic photolithography," Macromolecular Research, vol. 14, no. 2, pp. 155-165, 2006.

[118] M. J. Escuti and G. P. Crawford, "Mesoscale three dimensional lattices formed in polymer dispersed liquid crystals: a diamond-like face centered cubic," Molecular Crystals and Liquid Crystals, vol. 421, pp. 23-26, 2004.

[119] S. P. Gorkhali, J. Qi, and G. P. Crawford, "Electrically switchable mesoscale Penrose quasicrystal structure," Applied Physics Letters, vol. 86, no. 1, Article ID 011110, 3 pages, 2005.

[120] M. J. Escuti and G. P. Crawford, "Holographic photonic crystals," Optical Engineering, vol. 43, no. 9, pp. 1973-1987, 2004.

[121] J. Qi and G. P. Crawford, "Holographically formed polymer dispersed liquid crystal displays," Displays, vol. 25, no. 5, pp. 177-186, 2004.

[122] I. C. Khoo, Y. Z. Williams, B. Lewis, and T. Mallouk, “+photorefractive CdSe and gold nanowire-doped liquid crystals and polymer-dispersed-liquid-crystal photonic crystals," Molecular Crystals and Liquid Crystals, vol. 446, pp. 233-244, 2006.

[123] X. H. Sun, X. M. Tao, T. J. Ye, P. Xue, and Y.-S. Szeto, "Optics design and fabrication of 3D electrically switchable hexagonal photonic crystal," Applied Physics B, vol. 87, no. 1, pp. 65-69, 2007.

[124] X. Sun, X. Tao, T. Ye, P. Xue, and Y.-S. Szeto, "2D and 3D electrically switchable hexagonal photonic crystal in the ultraviolet range," Applied Physics B, vol. 87, no. 2, pp. 267271, 2007.

[125] V. Berger, O. Gauthier-Lafaye, and E. Costard, "Fabrication of a 2D photonic bandgap by a holographic method," Electronics Letters, vol. 33, no. 5, pp. 425-426, 1997.

[126] M. S. Li, S. T. Wu, and A. Y.-G. Fuh, "Superprism phenomenon based on holographic polymer dispersed liquid crystal films," Applied Physics Letters, vol. 88, no. 9, Article ID 091109, 3 pages, 2006.

[127] P. P. Markowicz, V. K. S. Hsiao, H. Tiryaki, et al., "Enhancement of third-harmonic generation in a polymer-dispersed liquid-crystal grating," Applied Physics Letters, vol. 87, no. 5, Article ID 051102, 3 pages, 2005.

[128] R. Jakubiak, T. J. Bunning, R. A. Vaia, L. V. Natarajan, and V. P. Tondiglia, "Electrically switchable, one-dimensional polymeric resonators from holographic photopolymerization: a new approach for active photonic bandgap materials," Advanced Materials, vol. 15, no. 3, pp. 241-244, 2003.

[129] D. E. Lucchetta, L. Criante, O. Francescangeli, and F. Simoni, "Wavelength flipping in laser emission driven by a switchable holographic grating," Applied Physics Letters, vol. 84, no. 6, pp. 837-839, 2004.
[130] D. E. Lucchetta, L. Criante, O. Francescangeli, and F. Simoni, "Light amplification by dye-doped holographic polymer dispersed liquid crystals," Applied Physics Letters, vol. 84, no. 24, pp. 4893-4895, 2004.

[131] D. E. Lucchetta, L. Criante, O. Francescangeli, and F. Simoni, "Compact lasers based on HPDLC gratings," Molecular Crystals and Liquid Crystals, vol. 441, pp. 97-109, 2005.

[132] S. J. Woltman, M. E. Sousa, H. Zhang, and G. P. Crawford, "Survey of switchable lasing configurations using structures of liquid crystal and polymer dispersions," in Liquid Crystal Materials, Devices, and Applications XI, vol. 6135 of Proceedings of SPIE, pp. 1-12, San Jose, Calif, USA, January 2006.

[133] R. Jakubiak, L. V. Natarajan, V. Tondiglia, et al., "Electrically switchable lasing from pyrromethene 597 embedded holographic-polymer dispersed liquid crystals," Applied Physics Letters, vol. 85, no. 25, pp. 6095-6097, 2004.

[134] G. S. He, T.-C. Lin, V. K. S. Hsiao, et al., "Tunable two-photon pumped lasing using a holographic polymerdispersed liquid-crystal grating as a distributed feedback element," Applied Physics Letters, vol. 83, no. 14, pp. 27332735, 2003.

[135] V. K. S. Hsiao, C. Lu, G. S. He, et al., "High contrast switching of distributed-feedback lasing in dye-doped HPDLC transmission grating structures," Optics Express, vol. 13, no. 10, pp. 3787-3794, 2005.

[136] S.-T. Wu, "Birefringence dispersions of liquid crystals," Physical Review A, vol. 33, no. 2, pp. 1270-1274, 1986.

[137] N. G. Sultanova, S. N. Kasarova, C. D. Ivanov, and I. D. Nikolov, "Refractive data of optical plastics for laser applications," in Holography 2005: International Conference on Holography, Optical Recording, and Processing of Information, vol. 6252 of Proceedings of SPIE, pp. 1-5, Varna, Bulgaria, May 2006.

[138] J. Qi, M. De Sarkar, G. T. Warren, and G. P. Crawford, "In situ shrinkage measurement of holographic polymer dispersed liquid crystals," Journal of Applied Physics, vol. 91, no. 8, p. 4795, 2002.

[139] K. Rai and A. K. Fontecchio, "Optimization of pressure response in HPDLC gratings based on polymer composition," Molecular Crystals and Liquid Crystals, vol. 450, no. 1, pp. 183-190, 2006.

[140] D. R. Cairns, C. C. Bowley, S. Danworaphong, et al., "Optical strain characteristics of holographically formed polymerdispersed liquid crystal films," Applied Physics Letters, vol. 77, no. 17, pp. 2677-2679, 2000.

[141] M. L. Ermold, K. Rai, and A. K. Fontecchio, "Hydrostatic pressure response of polymer-dispersed liquid crystal gratings," Journal of Applied Physics, vol. 97, no. 10, 4 pages, 2005.

[142] V. K. S. Hsiao, W. D. Kirkey, F. Chen, A. N. Cartwright, P. N. Prasad, and T. J. Bunning, "Organic solvent vapor detection using holographic photopolymer reflection gratings," Advanced Materials, vol. 17, no. 18, pp. 2211-2214, 2005.

[143] M. H. Keefe, J. L. O’Donnell, R. C. Bailey, S. T. Nguyen, and J. T. Hupp, "Permeable, microporous polymeric membrane materials constructed from discrete molecular squares," Advanced Materials, vol. 15, no. 22, pp. 1936-1939, 2003.

[144] M. Fally, I. Drevensek-Olenik, M. A. Ellabban, K. P. Pranzas, and J. Vollbrandt, "Colossal light-induced refractive-index modulation for neutrons in holographic polymer-dispersed liquid crystals," Physical Review Letters, vol. 97, no. 16, Article ID 167803, 4 pages, 2006.

[145] I. Drevensek-Olenik, M. A. Ellabban, M. Fally, K. P. Pranzas, and J. Vollbrandt, "Neutron diffraction from holographic 
polymer-dispersed liquid crystals," in Liquid Crystals and Applications in Optics, vol. 6587 of Proceedings of SPIE, pp. 1-6, Prague, Czech Republic, April 2007.

[146] A. K. Fontecchio, G. P. Crawford, C. He, and D. Content, "Performance improvements for switchable H-PDLC gratings using morphological studies," in Solar and Switching Materials, vol. 4458 of Proceedings of SPIE, pp. 230-239, San Diego, Calif, USA, August 2001.

[147] A. Ashkin, G. D. Boyd, J. M. Dziedzic, et al., "Opticallyinduced refractive index inhomogeneities in $\mathrm{LiNbO}_{3}$ and $\mathrm{LiTaO}_{3}$," Applied Physics Letters, vol. 9, no. 1, pp. 72-74, 1966.

[148] F. S. Chen, J. T. LaMacchia, and D. B. Fraser, "Holographic storage in lithium niobate," Applied Physics Letters, vol. 13, no. 7, pp. 223-225, 1968.

[149] F. S. Chen, "Optically induced change of refractive indices in $\mathrm{LiNbO}_{3}$ and $\mathrm{LiTaO}_{3}$," Journal of Applied Physics, vol. 40, no. 8, pp. 3389-3396, 1969.

[150] M. A. Ellabban, M. Fally, H. Uršič, and I. Drevensek-Olenik, "Holographic scattering in photopolymer-dispersed liquid crystals," Applied Physics Letters, vol. 87, no. 15, Article ID 151101, 3 pages, 2005.

[151] M. A. Ellabban, I. Drevensek-Olenik, M. Fally, and H. Uršič, "Effect of electric field and temperature on holographic scattering from holographic polymer-dispersed liquid crystals," Optical Materials, vol. 29, no. 11, pp. 1416-1422, 2007.

[152] S. Harbour, J. V. Kelly, T. Galstian, and J. T. Sheridan, "Optical birefringence and anisotropic scattering in acrylate based holographic polymer dispersed liquid crystals," Optics Communications, vol. 278, no. 1, pp. 28-33, 2007.

[153] M. R. B. Forshaw, "Explanation of the two-ring diffraction phenomenon observed by Moran and Kaminow," Applied Optics, vol. 13, no. 1, p. 2, 1974.

[154] M. A. Ellabban, M. Fally, M. Imlau, T. Woike, R. A. Rupp, and T. Granzow, "Angular and wavelength selectivity of parasitic holograms in cerium doped strontium barium niobate," Journal of Applied Physics, vol. 96, no. 12, pp. 6987-6993, 2004.

[155] T. Karasawa and Y. Taketomi, "Effects of material systems on the polarization behavior of holographic polymer dispersed liquid crystal gratings," Japanese Journal of Applied Physics, vol. 36, no. 10, pp. 6388-6392, 1997.

[156] M. S. Park, Y. H. Cho, B. K. Kim, and J. S. Jang, "Fabrication of reflective holographic gratings with polyurethane acrylate (PUA)," Current Applied Physics, vol. 2, no. 3, pp. 249-252, 2002.

[157] G. T. Warren, M. DeSarkar, J. Qi, and G. P. Crawford, "In-situ spectroscopy of holographically formed polymer dispersed liquid crystal materials for high performance reflective display applications," in Proceedings of the International Symposium Digest of Technical Papers (SID '01), vol. 3, pp. 866-869, San Jose, Calif, USA, June 2001.

[158] E. Y. Shin, J. A. Jung, E. H. Kim, and B. K. Kim, "Holographic polymer-dispersed liquid crystal fabrication under electric field," Polymer International, vol. 54, no. 6, pp. 922-925, 2005.

[159] J. Colegrove, T. Fiske, A. Lewis, et al., "The effect of monomer functionality on HPDLC performance and aging," in Proceedings of the International Symposium Digest of Technical Papers (SID '01), vol. 32, pp. 962-965, San Jose, Calif, USA, June 2001. 

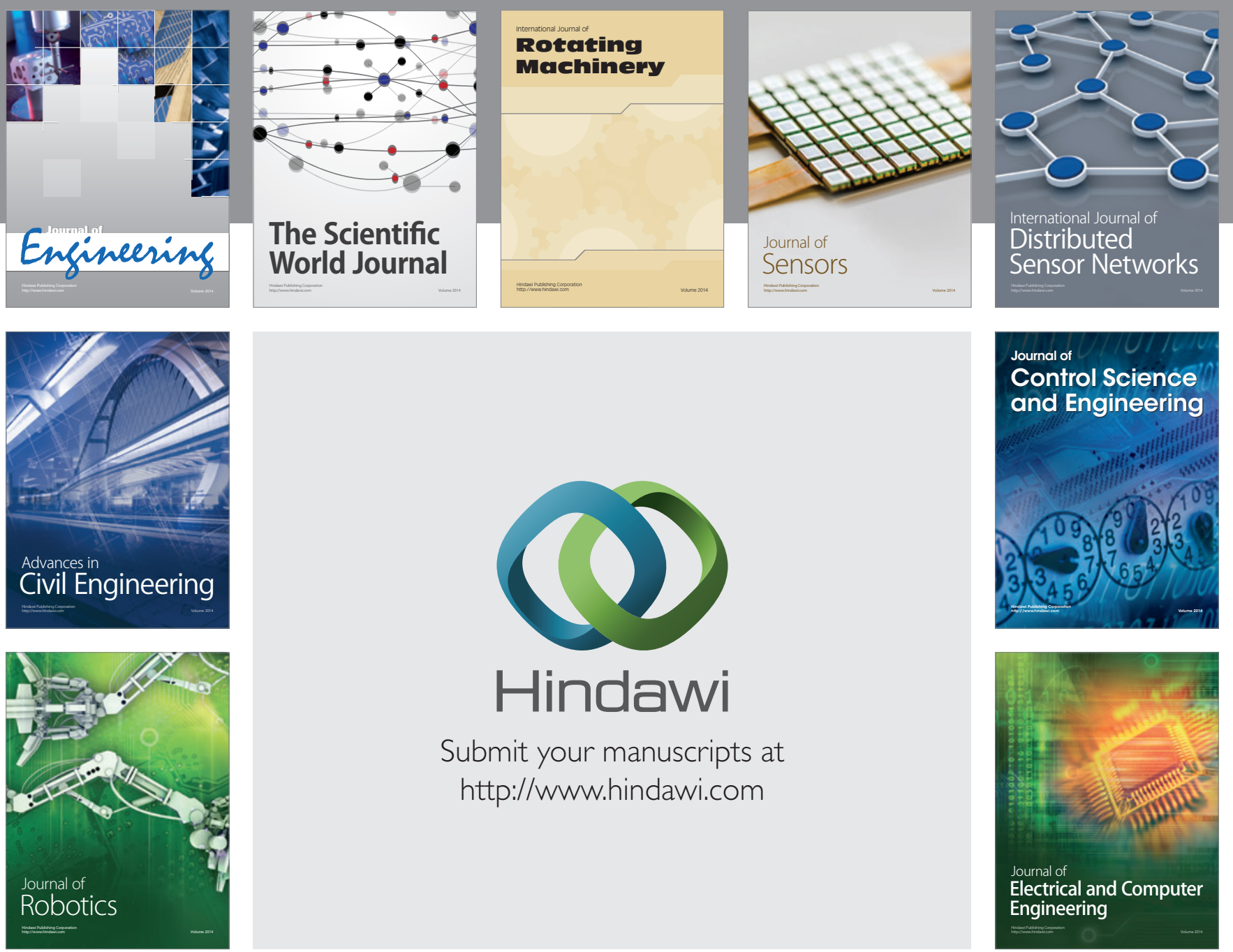

Submit your manuscripts at

http://www.hindawi.com
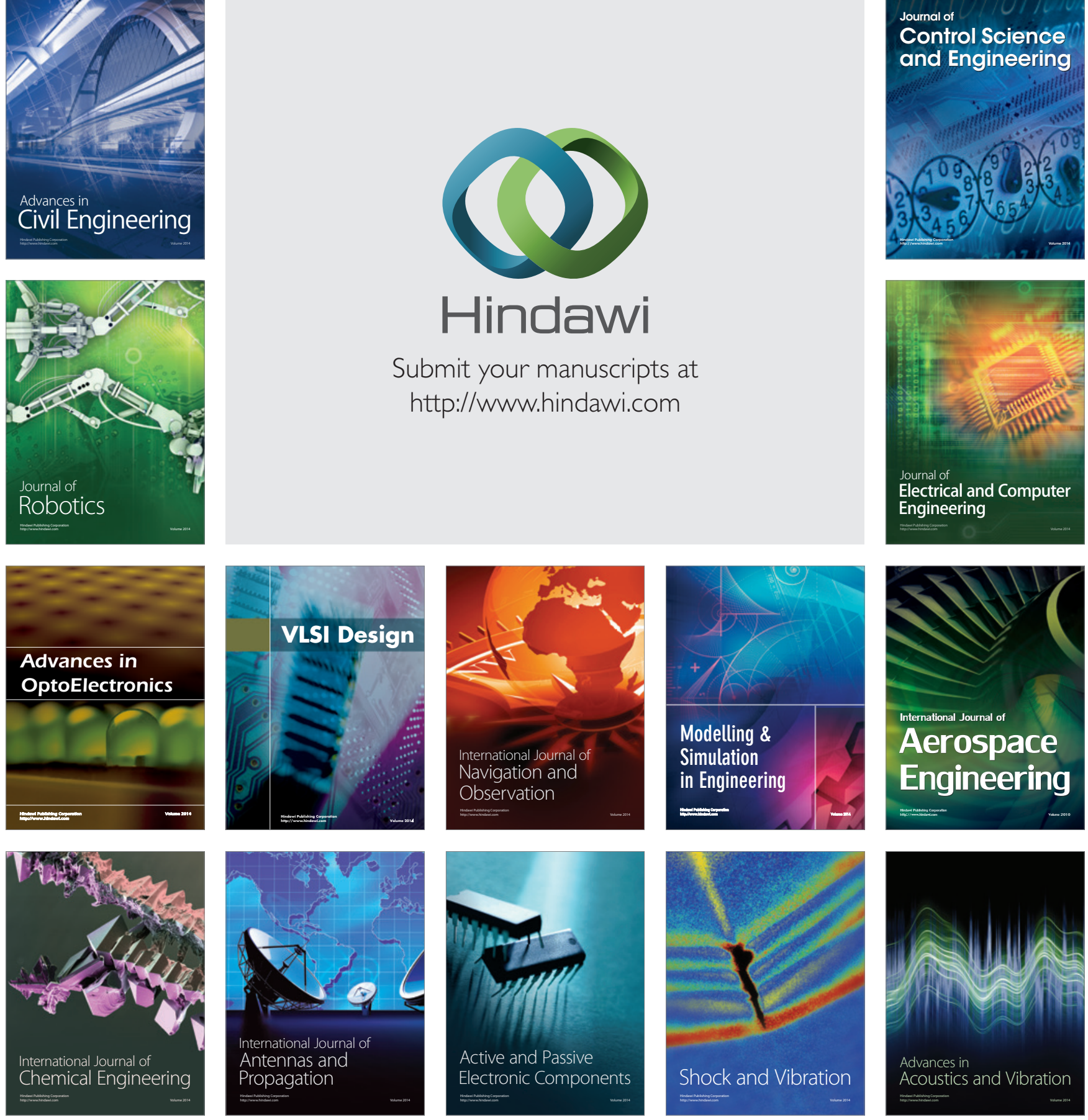